\title{
Deprotonated Salicylaldehyde as Visible Light Photocatalyst
}

Yan-Jun Zhuang ${ }^{\dagger}$, Jian-Ping $\mathrm{Qu}^{\dagger}$, Yan-Biao Kang ${ }^{\dagger}, *$

$\dagger$ Department of Chemistry, University of Science and Technology of China, Hefei, Anhui 230026, China

$\$$ Institute of Advanced Synthesis, School of Chemistry and Molecular Engineering, Nanjing Tech University, Nanjing 211816, China

E-mail: ybkang@ustc.edu.cn

\section{Contents}

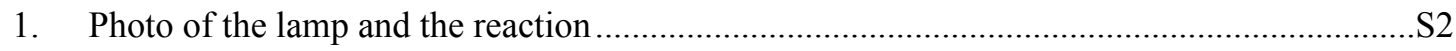

2 Measurement of fluorescence quantum yield $(\Phi)$............................................................. 3

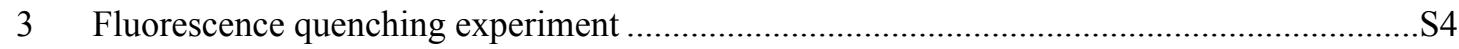

4 Calculation of apparent quantum efficiency (A. Q. E.).......................................................

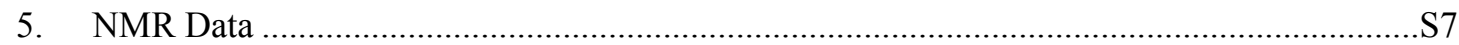


1. Photo of the lamp and the reaction

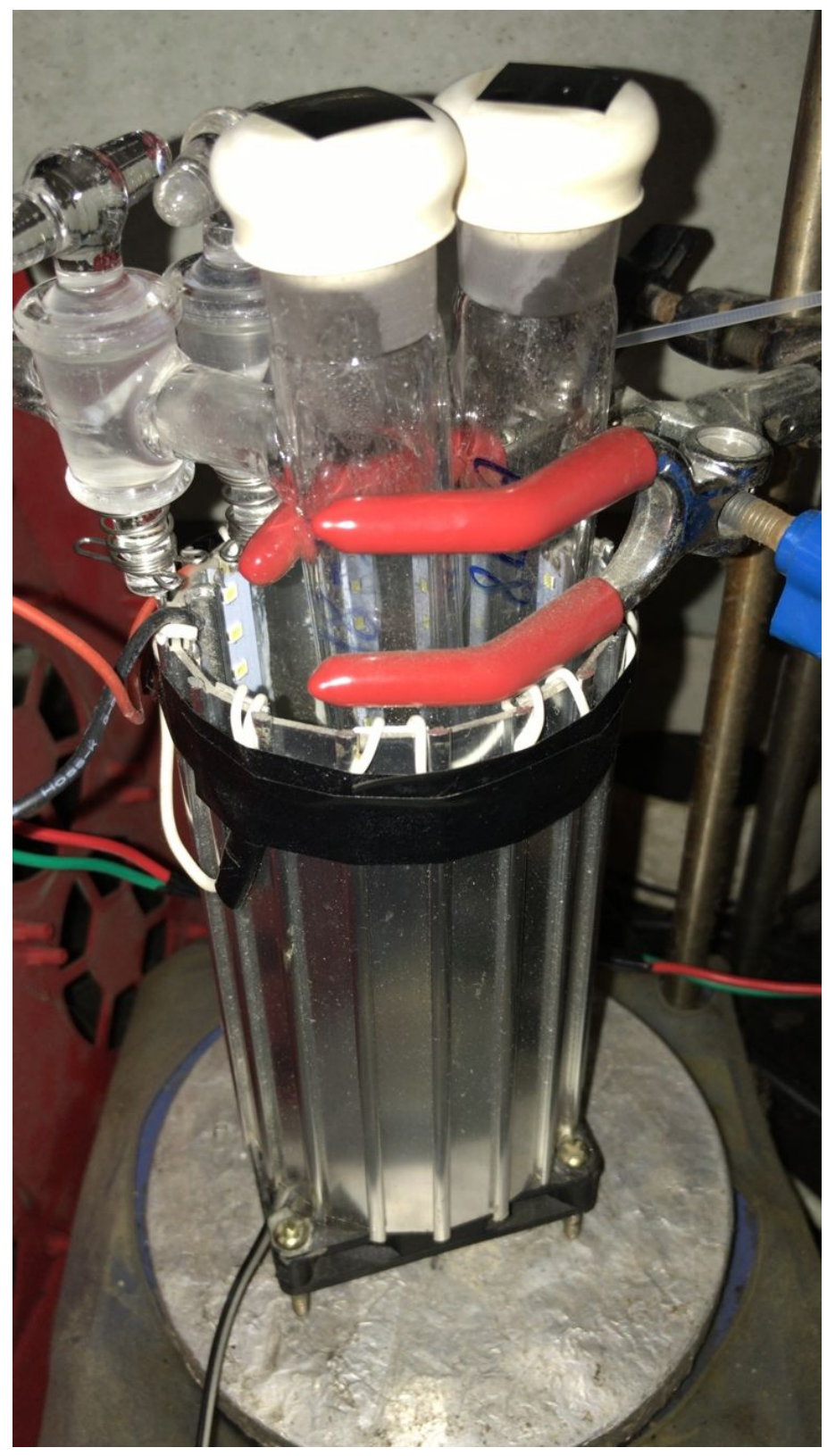

Figure S1. Photo of the lamp and the reaction. 


\section{Measurement of fluorescence quantum yield $(\Phi)$}

$\Phi$ was measured by the optical dilute method with a standard of quinine sulfate $\left(\Phi_{\mathrm{r}}=0.55\right.$, quinine in $0.05 \mathrm{M}$ sulfuric acid) calculated by:

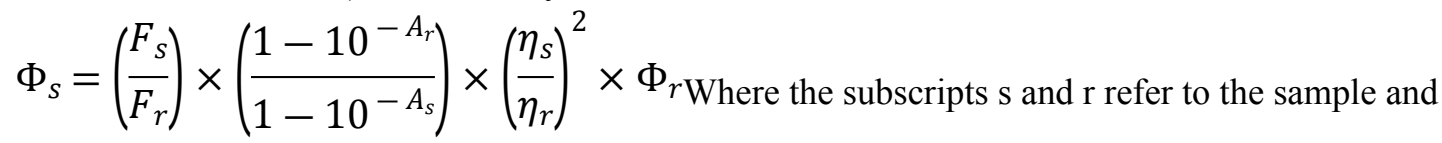
reference standard solution respectively; $\mathrm{F}$ is the integrated intensity; $\mathrm{A}$ is the absorbance at the excitation wavelength; $\eta$ is the refractive index of the solutions. Error for $\Phi$ values $( \pm 10 \%)$ are estimated. 


\section{Fluorescence quenching experiment}
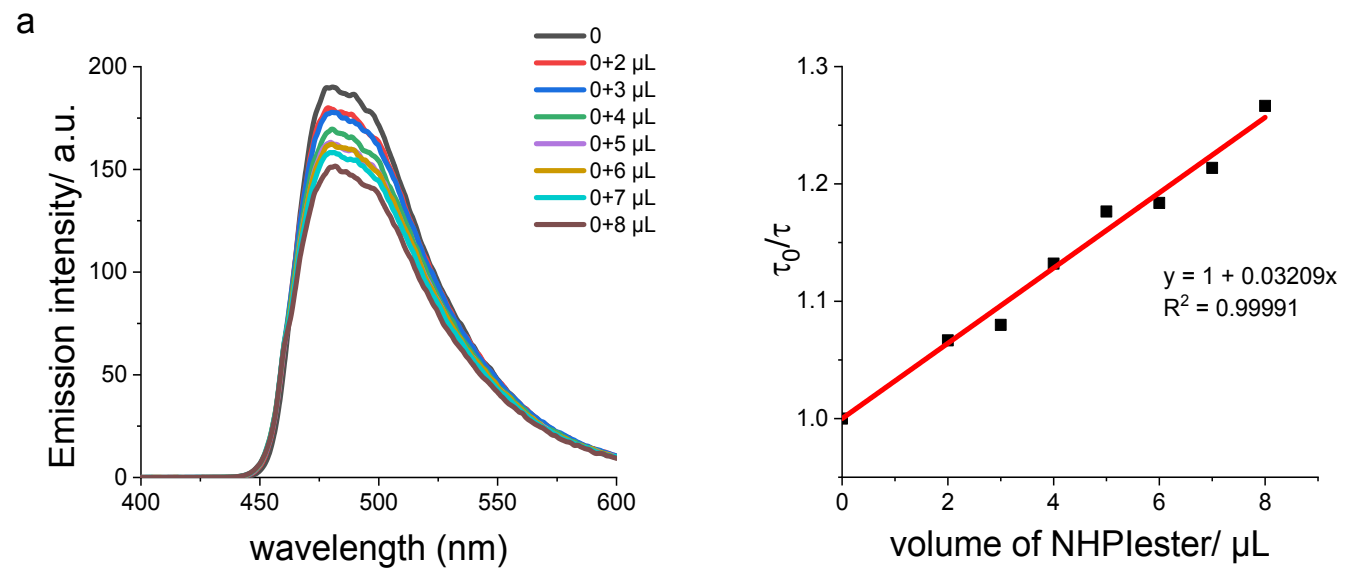

Figure S2. Fluorescence quenching effect of catalyst by NHPI ester in DMSO.

A stock solution of salicylaldehyde $(0.10 \mathrm{M}$, in dry DMSO) was prepared in a volumetric flask and diluted by dry DMSO to $1.0 \mathrm{mM}$ for the quenching experiment. Then $\mathrm{K}_{3} \mathrm{PO}_{4}$ was added as the base. The fluorescence measurement was monitored at least $1 \mathrm{~h}$ after the addition of base. A quartz cuvette $(1 \mathrm{~cm} \times 1 \mathrm{~cm} \times 3 \mathrm{~cm})$ was filled with the abovementioned $1.0 \mathrm{mM}$ DMSO solution $(3 \mathrm{~mL})$ and its fluorescence was recorded with excitation at $458 \mathrm{~nm}$ in the spectrometer. Quenching experiments were performed with the injection of $1 \mu \mathrm{L}$ solution of NHPI ester in DMSO (0.1 M) respectively by auto-pipette.
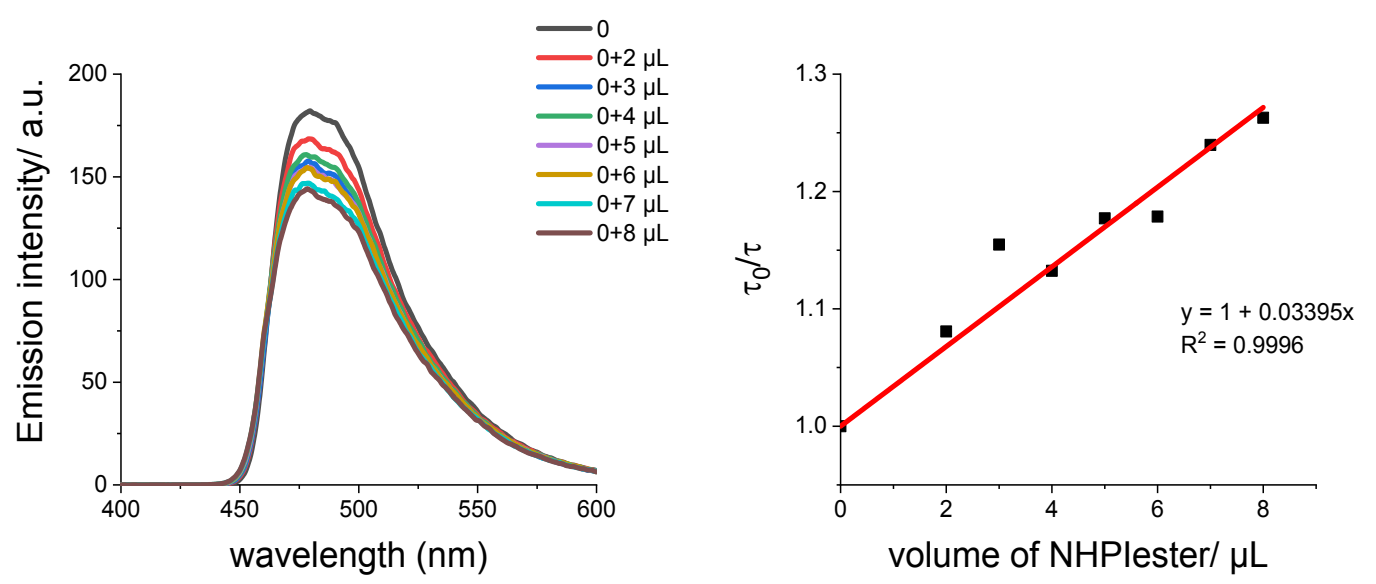

Figure S3. Fluorescence quenching effect of catalyst by NHPI ester in DMAc.

A stock solution of salicylaldehyde $(0.10 \mathrm{M}$, in dry DMAc) was prepared in a volumetric flask and diluted by dry DMAc to $1.0 \mathrm{mM}$ for the quenching experiment. Then $\mathrm{K}_{3} \mathrm{PO}_{4}$ was added as the base. The fluorescence measurement was monitored at least $1 \mathrm{~h}$ after the addition of base. A quartz cuvette $(1 \mathrm{~cm} \times 1 \mathrm{~cm} \times 3 \mathrm{~cm})$ was filled with the abovementioned $1.0 \mathrm{mM}$ DMAc solution $(3 \mathrm{~mL})$ and its fluorescence was recorded with excitation at $458 \mathrm{~nm}$ in the spectrometer.

Quenching experiments were performed with the injection of $1 \mu \mathrm{L}$ solution of NHPI ester in DMAc $(0.1 \mathrm{M})$ respectively by auto-pipette. 

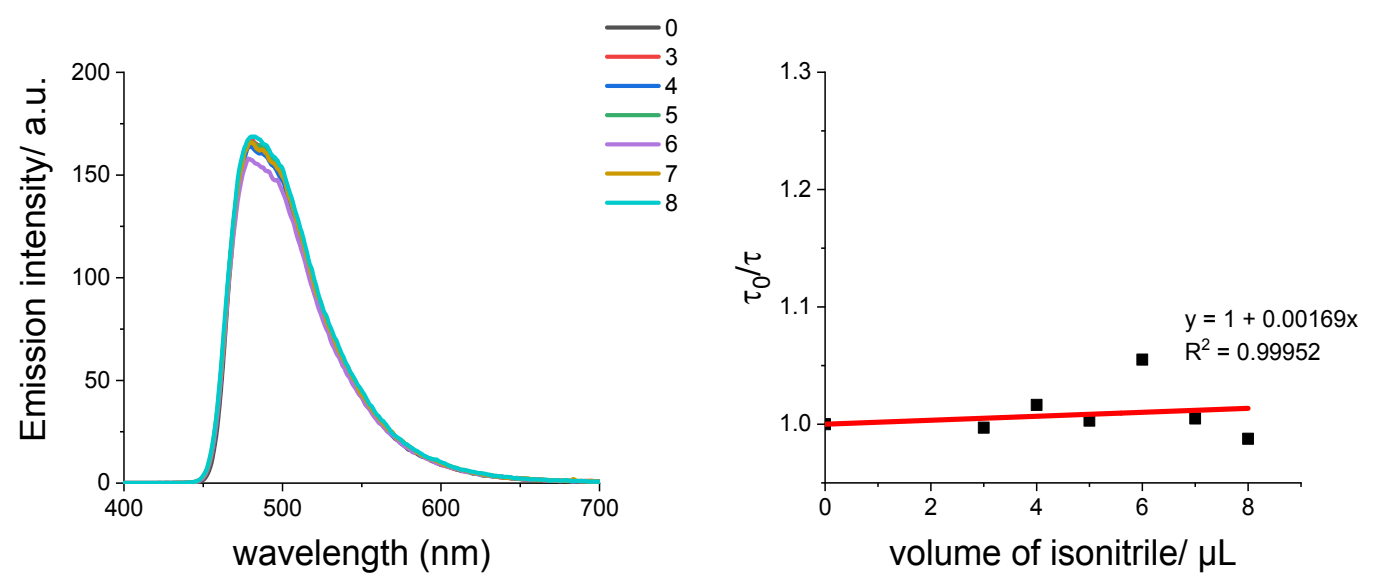

Figure S4. Fluorescence quenching effect of catalyst by isonitrile in DMSO.

A stock solution of salicylaldehyde $(0.10 \mathrm{M}$, in dry DMSO) was prepared in a volumetric flask and diluted by dry DMSO to $1.0 \mathrm{mM}$ for the quenching experiment. Then $\mathrm{K}_{3} \mathrm{PO}_{4}$ was added as the base. The fluorescence measurement was monitored at least $1 \mathrm{~h}$ after the addition of base. A quartz cuvette $(1 \mathrm{~cm} \times 1 \mathrm{~cm} \times 3 \mathrm{~cm})$ was filled with the abovementioned $1.0 \mathrm{mM}$ DMSO solution $(3 \mathrm{~mL})$ and its fluorescence was recorded with excitation at $458 \mathrm{~nm}$ in the spectrometer.

Quenching experiments were performed with the injection of $1 \mu \mathrm{L}$ solution of isonitrile in DMSO $(0.1 \mathrm{M})$ respectively by auto-pipette. 


\section{Calculation of apparent quantum efficiency (A. Q. E.)}

In principle, it takes one photon to excite one deprotonated salicylaldehyde molecule to generate one alkyl radical. The energy of one photon $\left(\mathrm{E}_{\text {photon }}\right)$ with wavelength of $\lambda_{\text {inc }}(\mathrm{nm})$ is calculated using the following equation:

$$
E_{\text {photo }}=\frac{h c}{\lambda_{\text {inc }}}
$$

\begin{tabular}{|c|c|c|c|c|c|c|}
\hline$\lambda_{\text {inc }}(\mathrm{nm})$ & 405 & 430 & 450 & 520 & 595 & 620 \\
\hline$E_{\text {photo }}(\mathrm{J})$ & $4.9 \times 10^{-19}$ & $4.6 \times 10^{-19}$ & $4.4 \times 10^{-19}$ & $3.8 \times 10^{-19}$ & $3.3 \times 10^{-19}$ & $3.2 \times 10^{-19}$ \\
\hline
\end{tabular}

Where $h(\mathrm{~J} \bullet \mathrm{s})$ is Planck's constant, $c\left(\mathrm{~m} \bullet \mathrm{s}^{-1}\right)$ is the speed of light and $\lambda_{\text {inc }}(\mathrm{m})$ is the wavelength of the incident light. And the total energy of the incident monochromatic light $\left(E_{\text {total }}\right)$ is calculated using the following equation:

\begin{tabular}{|c|c|c|c|c|c|c|}
\hline$\lambda_{\text {inc }}(\mathrm{nm})$ & 405 & 430 & 450 & 520 & 595 & 620 \\
\hline$E_{\text {total }}(\mathrm{J})$ & 112.32 & 112.32 & 95.04 & 86.40 & 64.80 & 60.48 \\
\hline
\end{tabular}

Where $P\left(\mathrm{~W} \cdot \mathrm{cm}^{-2}\right)$ is the power density of the incident light, $S\left(\mathrm{~cm}^{2}\right)$ is the irradiation area and $t$

(s) is the photoreaction tiom. The total number of the incident can be obtained through the following equation:

Number of incident photons $=\frac{E_{\text {total }}}{E_{\text {photo }}}$
\begin{tabular}{|c|c|c|c|c|c|c|}
\hline$\lambda_{\text {inc }}(\mathrm{nm})$ & 405 & 430 & 450 & 520 & 595 & 620 \\
\hline $\begin{array}{c}\text { Number of incident photons } \\
\text { (mmol) }\end{array}$ & 0.38 & 0.41 & 0.36 & 0.38 & 0.33 & 0.31 \\
\hline
\end{tabular}

Because it is difficult determine the number of reacted electrons via experimental methods, as a result, the apparent quantum yield (A. Q. Y.) is defined as follows:

A. Q. Y. $=\frac{\text { Number of product }}{\text { Number of incident photos }} \times 100 \%$
\begin{tabular}{|c|c|c|c|c|c|c|}
\hline$\lambda_{\text {inc }}(\mathrm{nm})$ & 405 & 430 & 450 & 520 & 595 & 620 \\
\hline A.Q.Y. (\%) & 44.2 & 41.5 & 47.2 & 0 & 0 & 0 \\
\hline
\end{tabular}




\section{NMR Data}

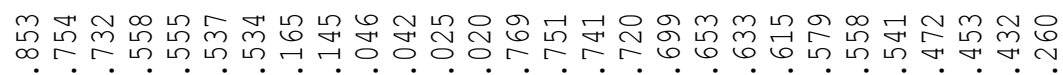

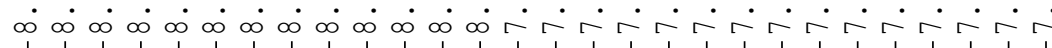

PIn
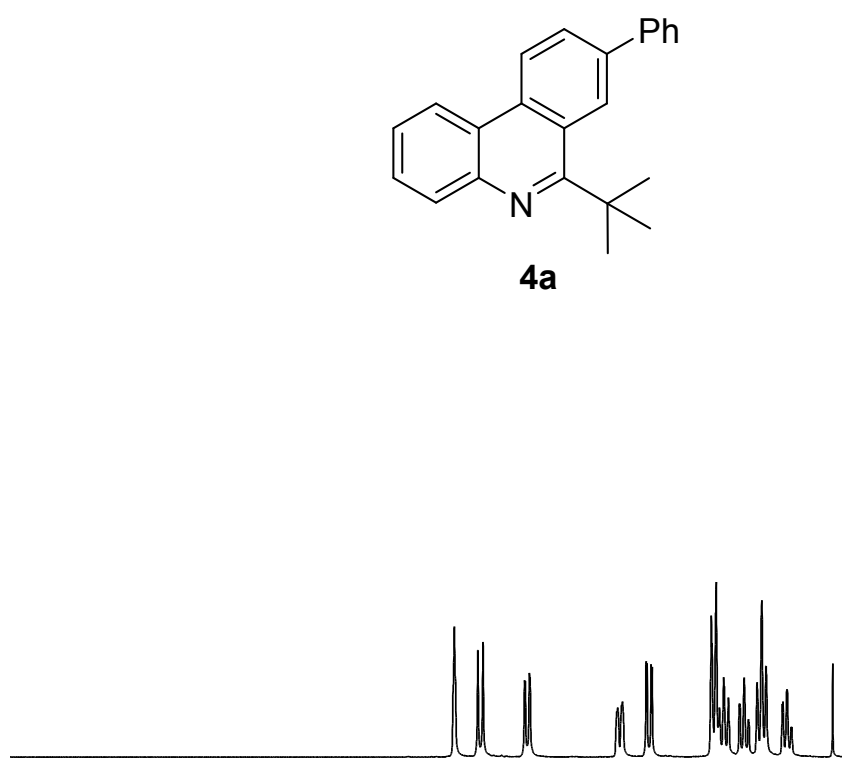

10

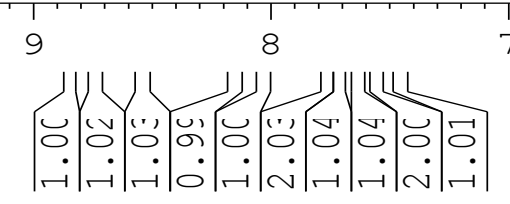

6

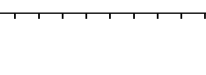

3

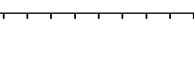

2

$\left|\begin{array}{c}3 \\ \hdashline \\ \dot{ }\end{array}\right|$

Figure S5. ${ }^{1} \mathrm{H}$ NMR spectrum of $\mathbf{4 a}\left(\mathrm{CDCl}_{3}, 400 \mathrm{MHz}\right)$. 

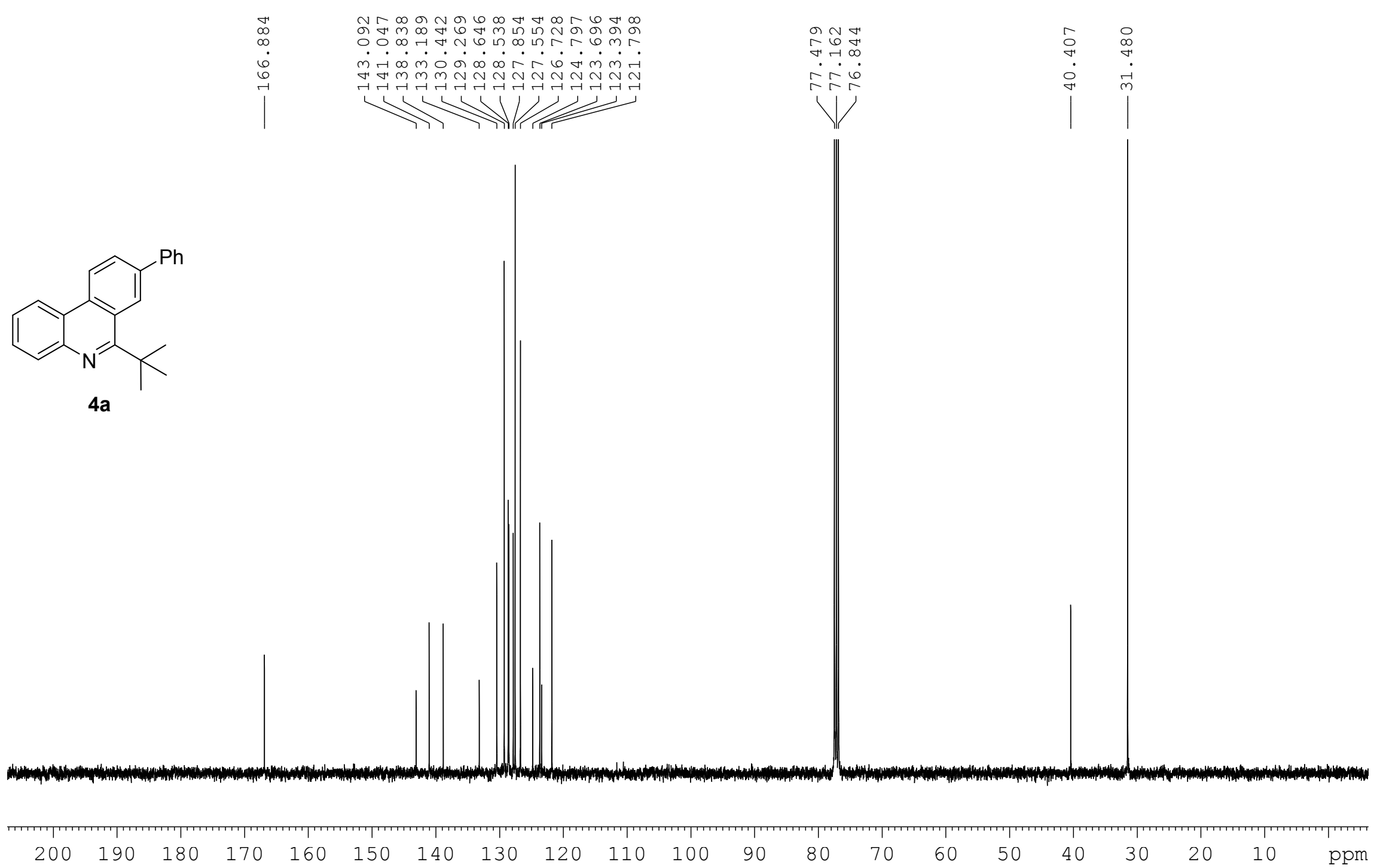

Figure S6. ${ }^{13} \mathrm{C}$ NMR spectrum of $\mathbf{4 a}\left(\mathrm{CDCl}_{3}, 100 \mathrm{MHz}\right)$. 


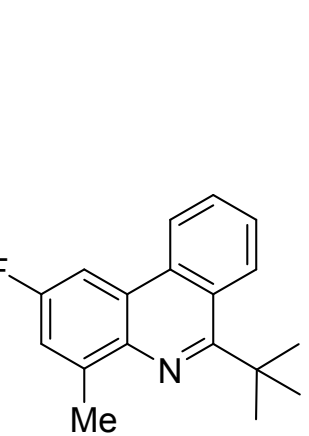

$4 b$

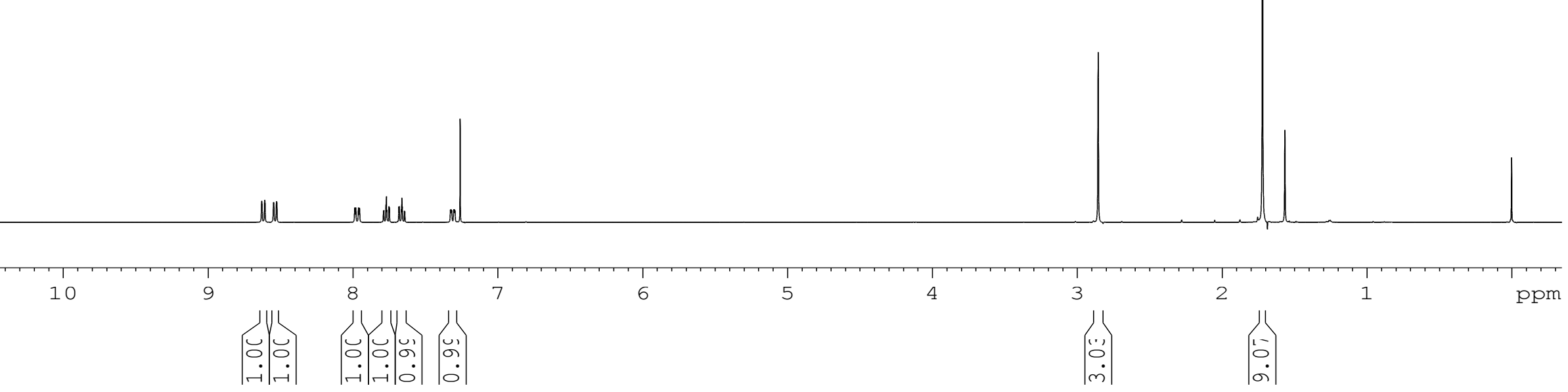

Figure S7. ${ }^{1} \mathrm{H}$ NMR spectrum of $\mathbf{4 b}\left(\mathrm{CDCl}_{3}, 400 \mathrm{MHz}\right)$ 

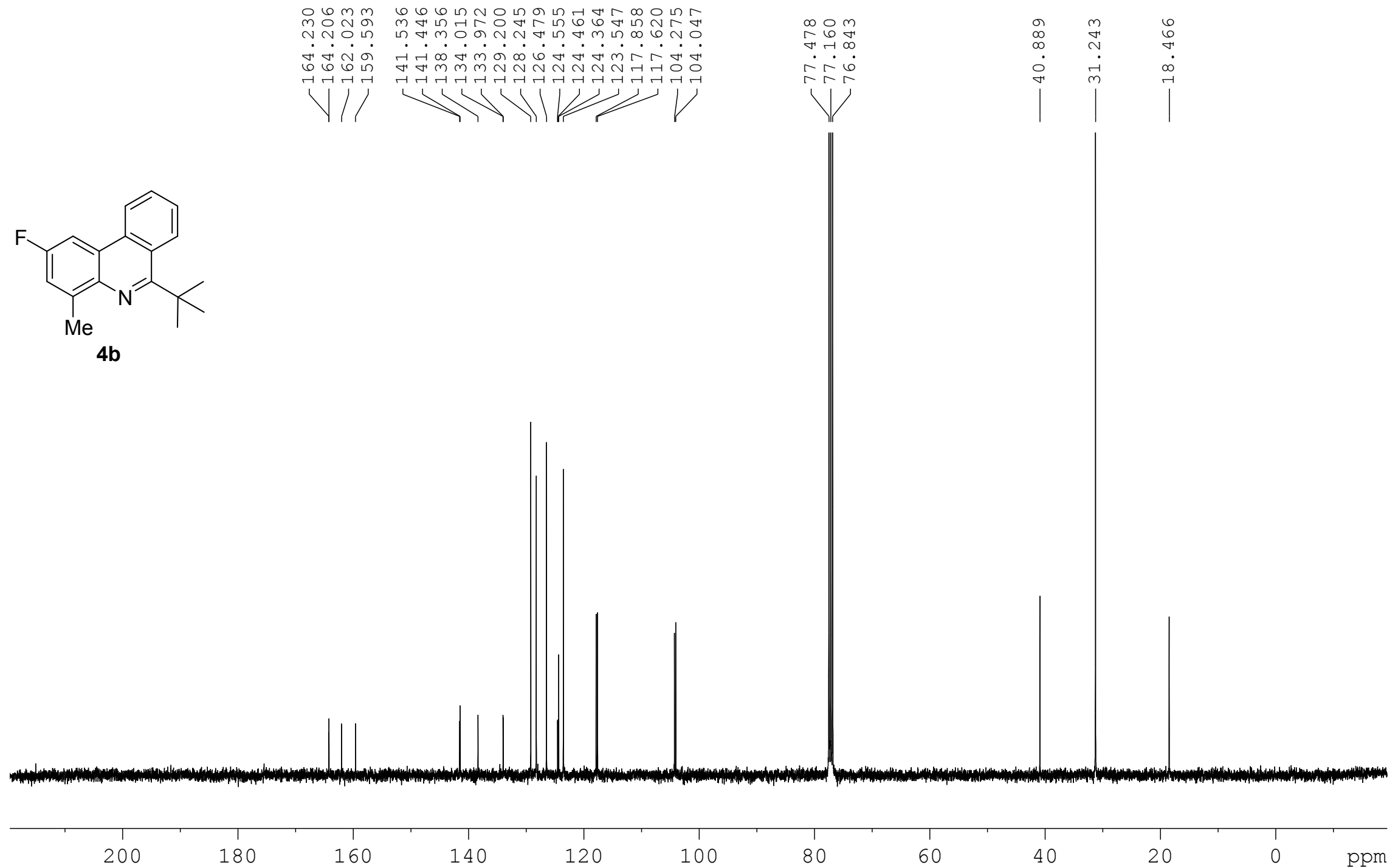

Figure S8. ${ }^{13} \mathrm{C}$ NMR spectrum of $\mathbf{4 b}\left(\mathrm{CDCl}_{3}, 100 \mathrm{MHz}\right)$. 


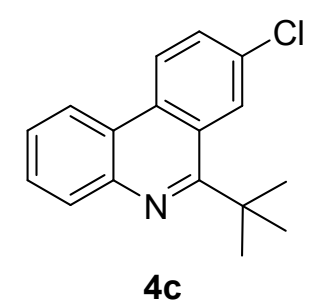

$4 c$

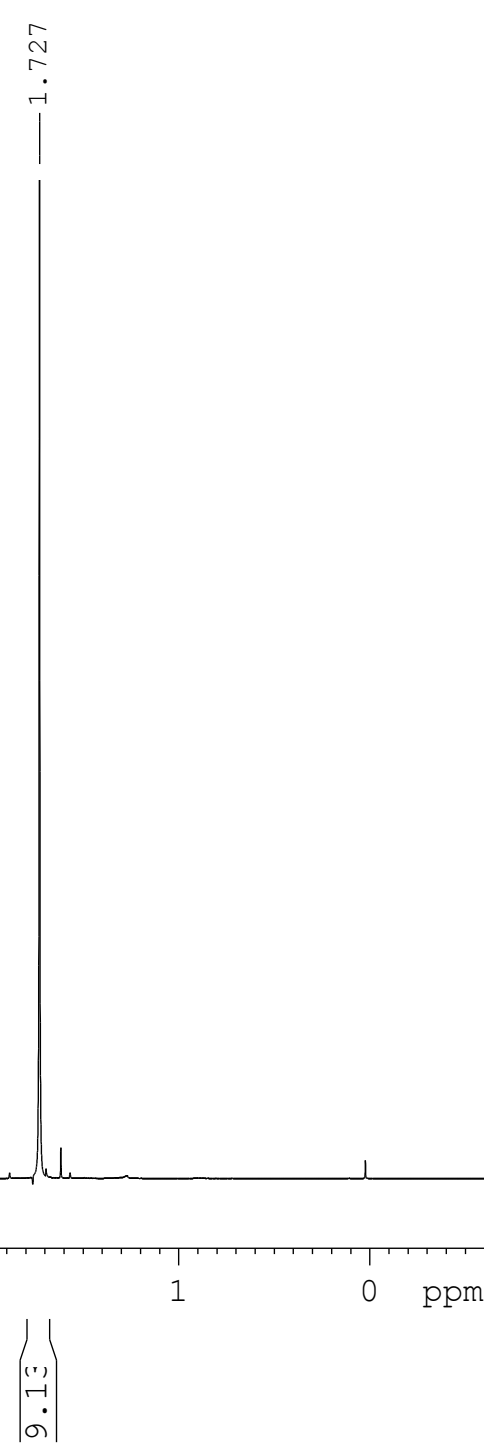

Figure S9. ${ }^{1} \mathrm{H}$ NMR spectrum of $\mathbf{4 c}\left(\mathrm{CDCl}_{3}, 400 \mathrm{MHz}\right)$. 

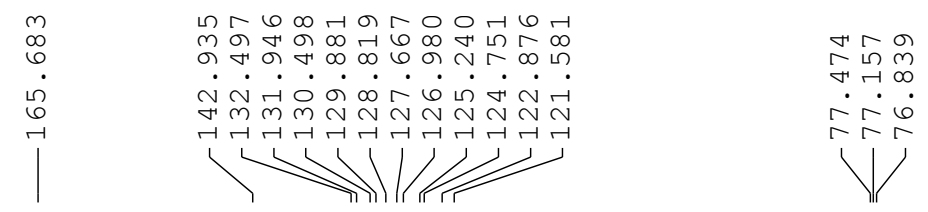

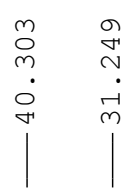

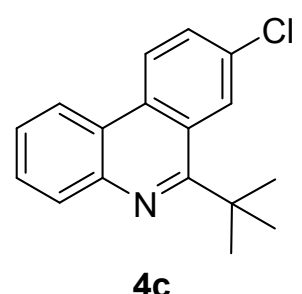

4c

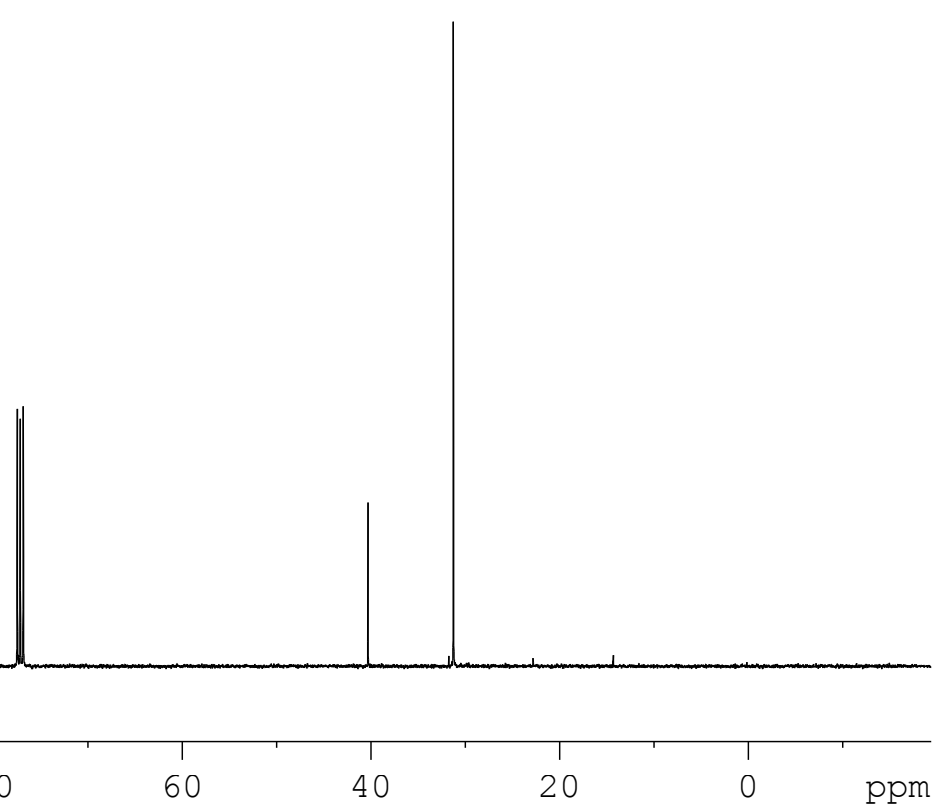

Figure S10. ${ }^{13} \mathrm{C}$ NMR spectrum of $4 \mathbf{c}\left(\mathrm{CDCl}_{3}, 100 \mathrm{MHz}\right)$. 

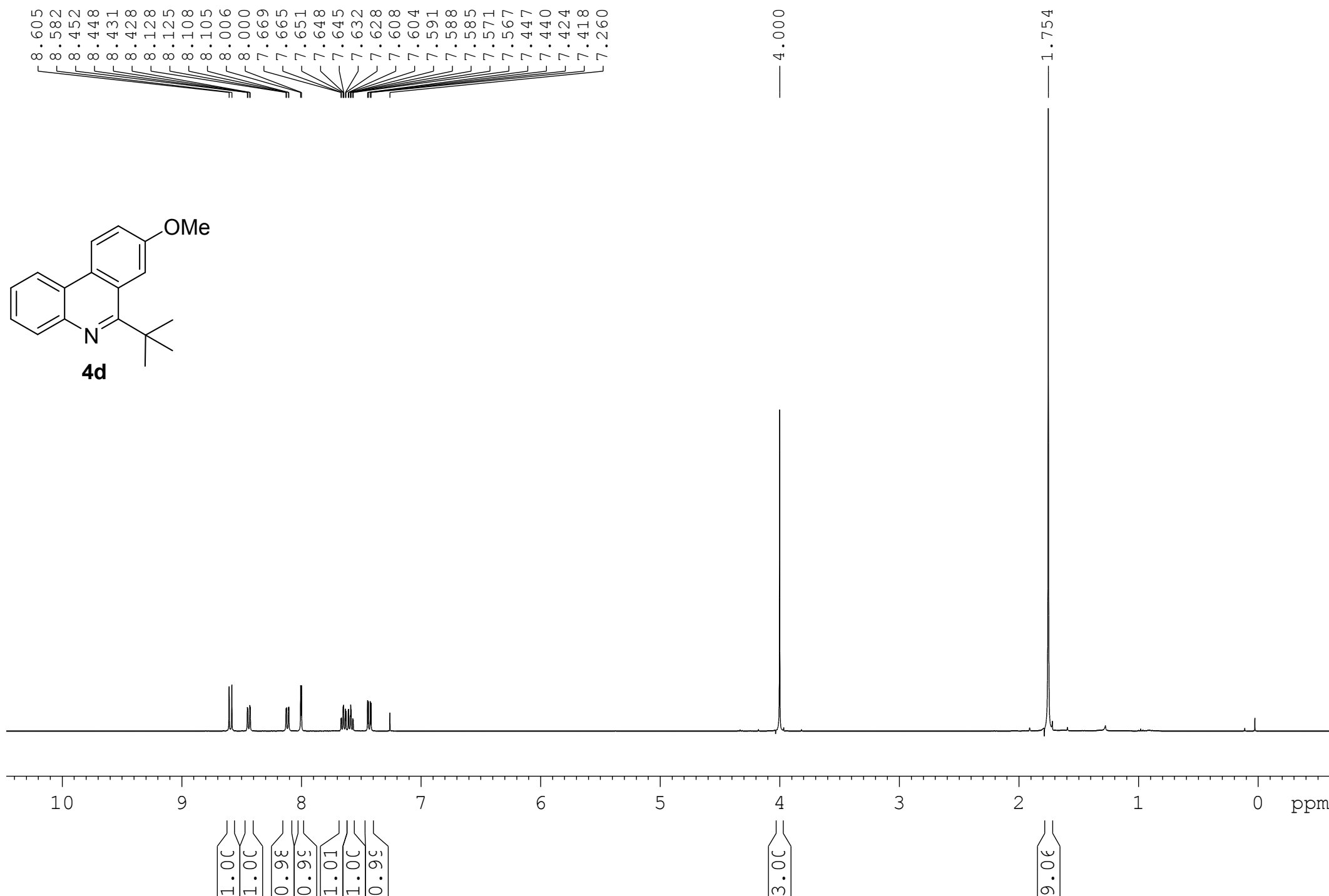

Figure S11. ${ }^{1} \mathrm{H}$ NMR spectrum of $4 \mathbf{d}\left(\mathrm{CDCl}_{3}, 400 \mathrm{MHz}\right)$. 


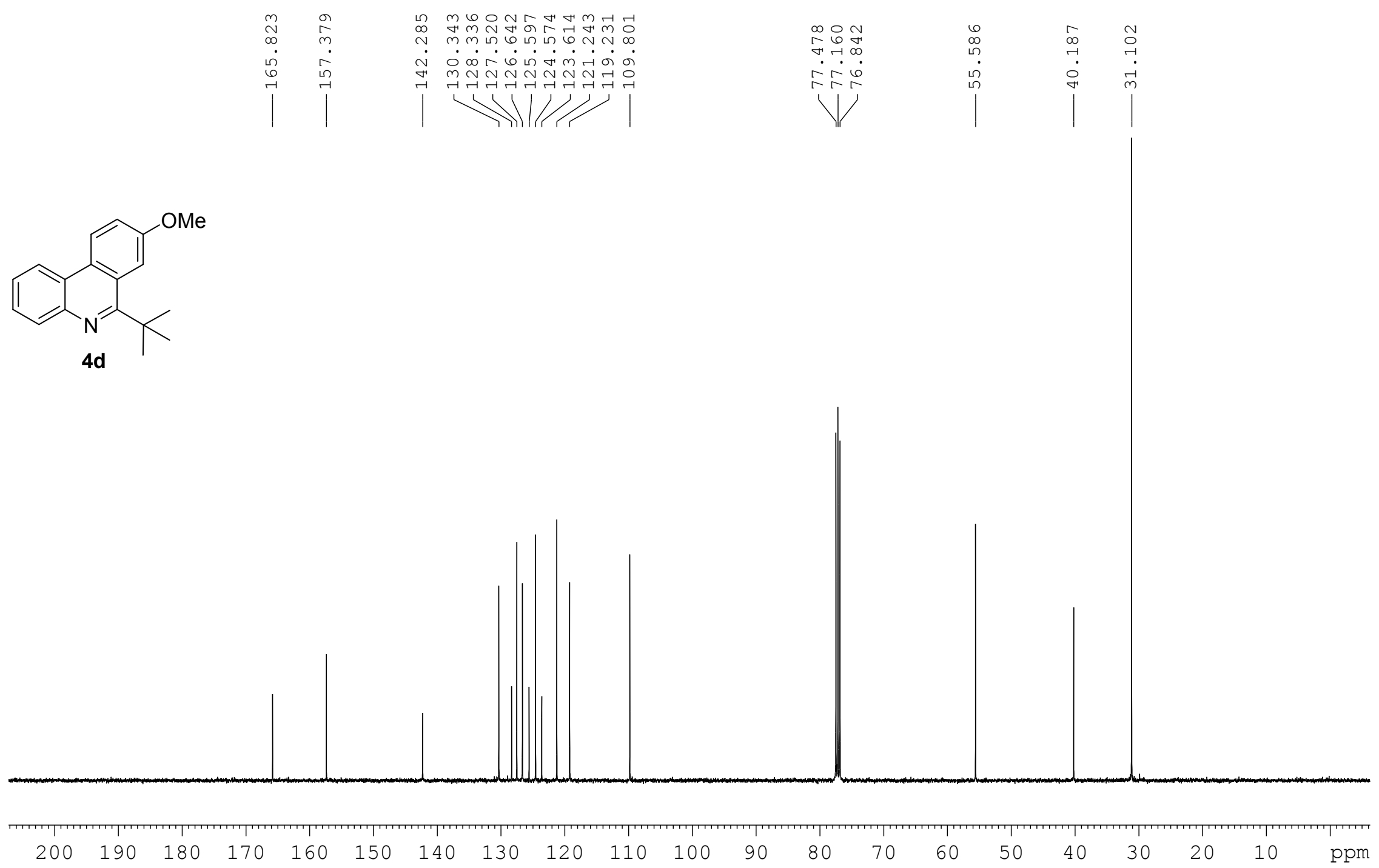

Figure S12. ${ }^{13} \mathrm{C}$ NMR spectrum of $\mathbf{4 d}\left(\mathrm{CDCl}_{3}, 100 \mathrm{MHz}\right)$. 


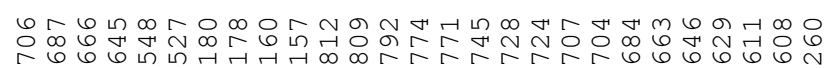

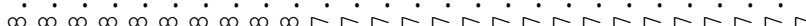

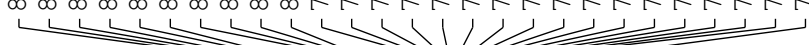

inim

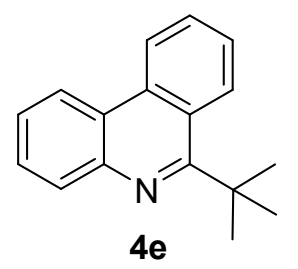

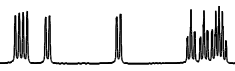

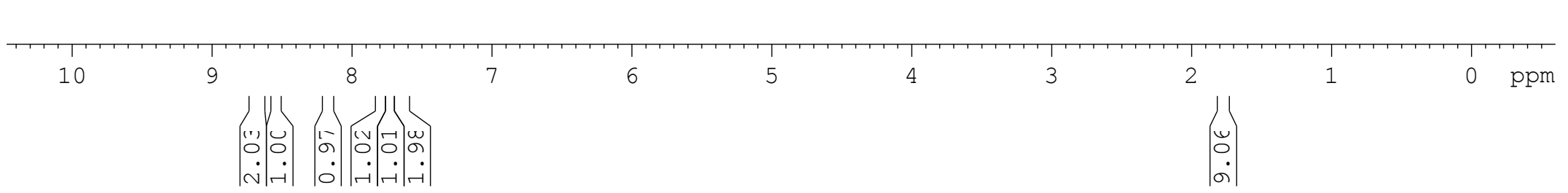


Figure S13. ${ }^{1} \mathrm{H}$ NMR spectrum of $4 \mathbf{e}\left(\mathrm{CDCl}_{3}, 400 \mathrm{MHz}\right)$
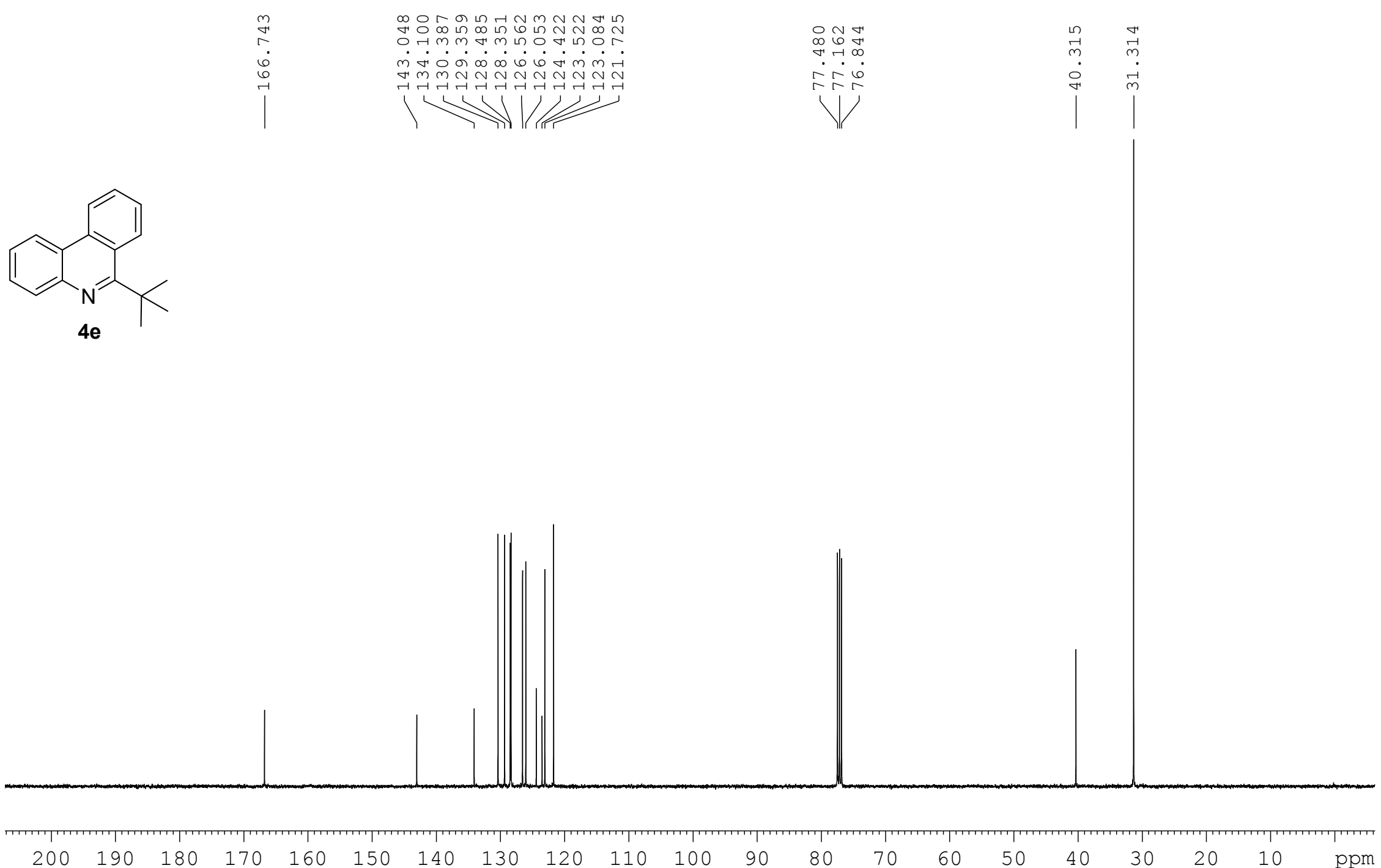
Figure S14. ${ }^{13} \mathrm{C}$ NMR spectrum of $4 \mathrm{e}\left(\mathrm{CDCl}_{3}, 100 \mathrm{MHz}\right)$.

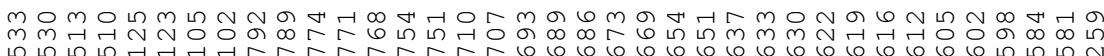

$\underbrace{\infty 0000000000}$

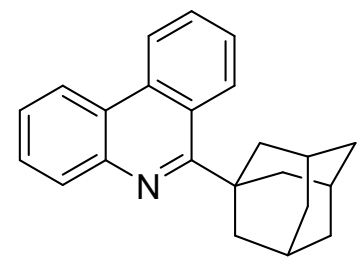

$4 f$
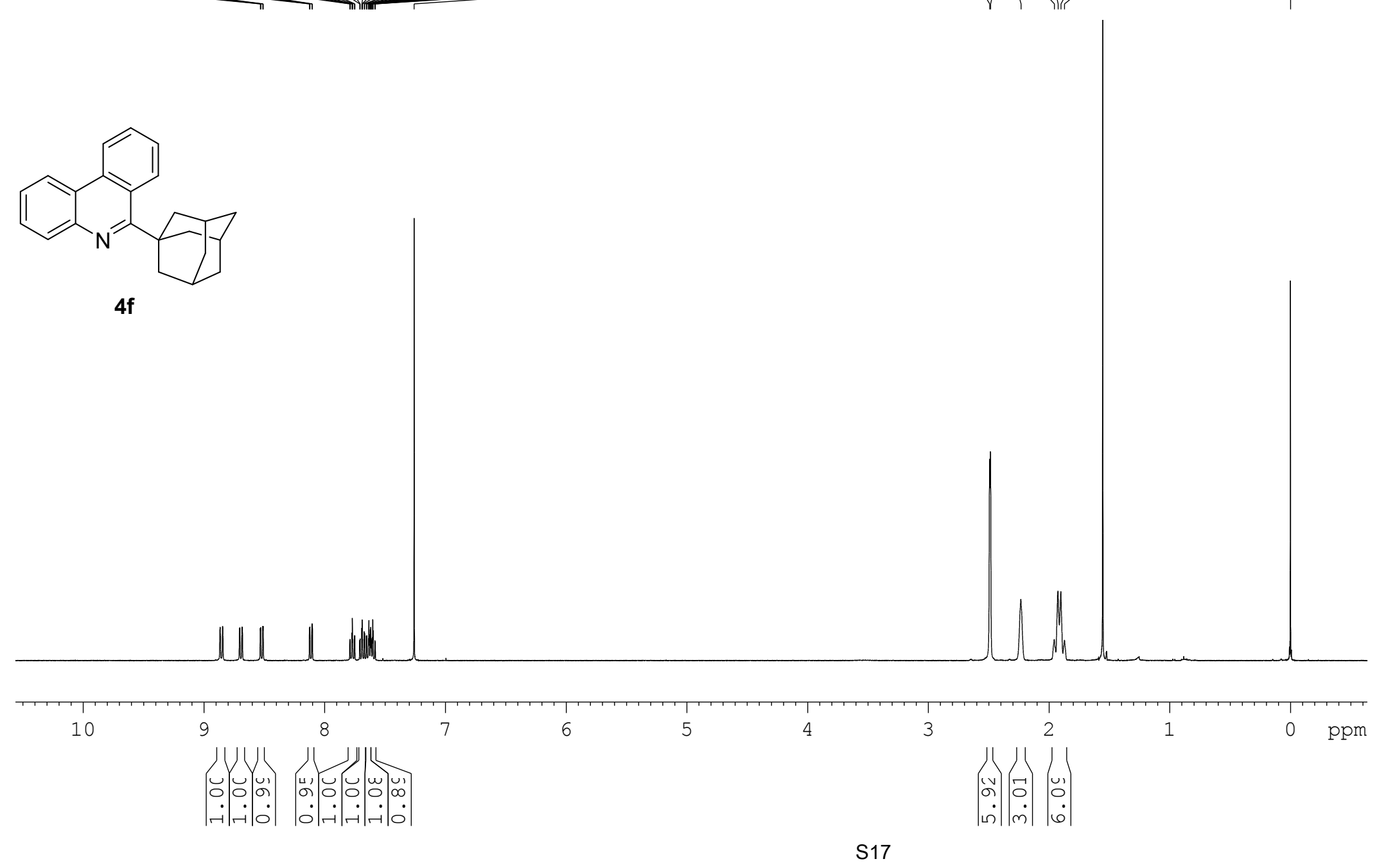
Figure S15. ${ }^{1} \mathrm{H}$ NMR spectrum of $\mathbf{4 f}\left(\mathrm{CDCl}_{3}, 400 \mathrm{MHz}\right)$ 


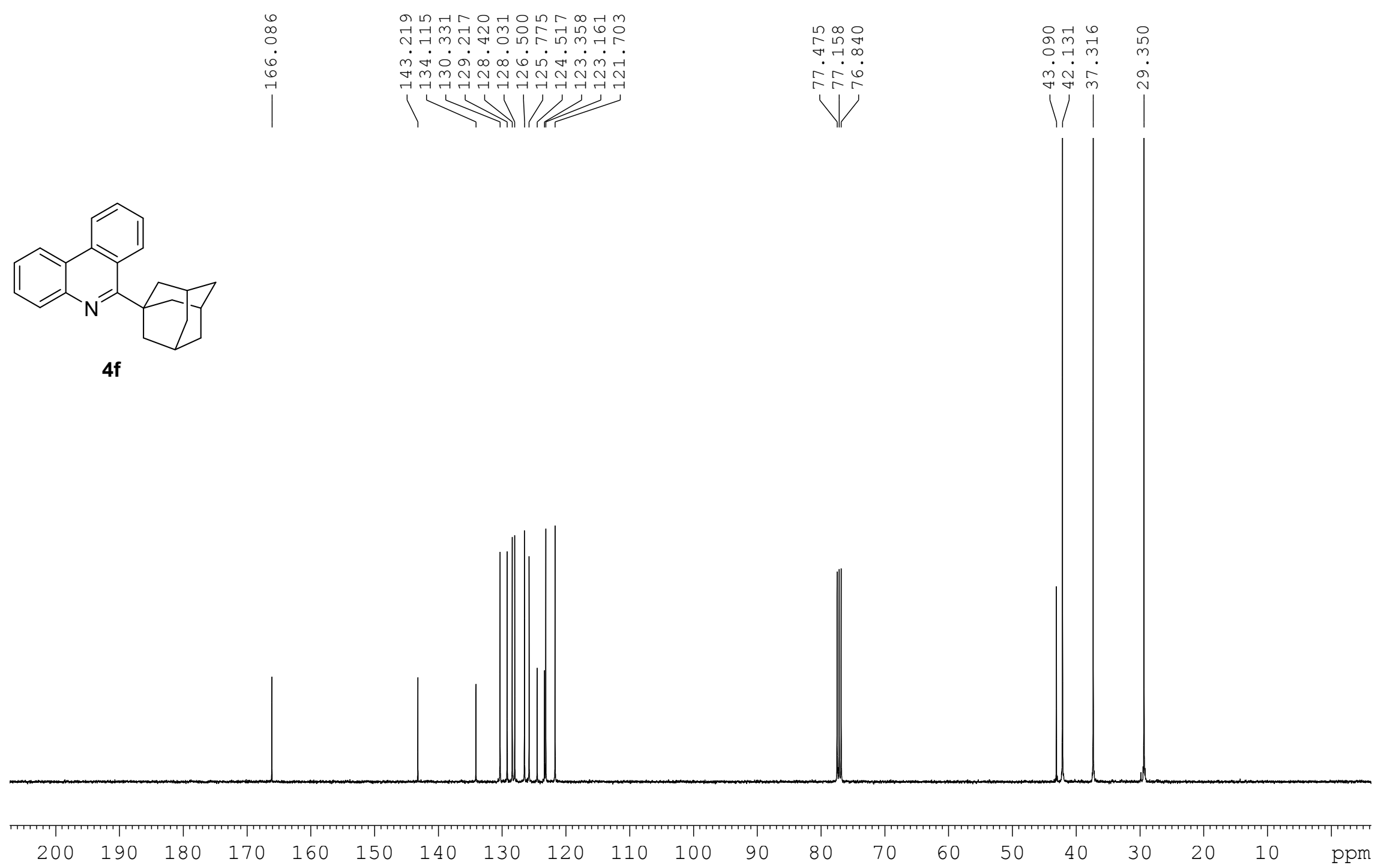

Figure S16. ${ }^{13} \mathrm{C}$ NMR spectrum of $\mathbf{4 f}\left(\mathrm{CDCl}_{3}, 100 \mathrm{MHz}\right)$. 

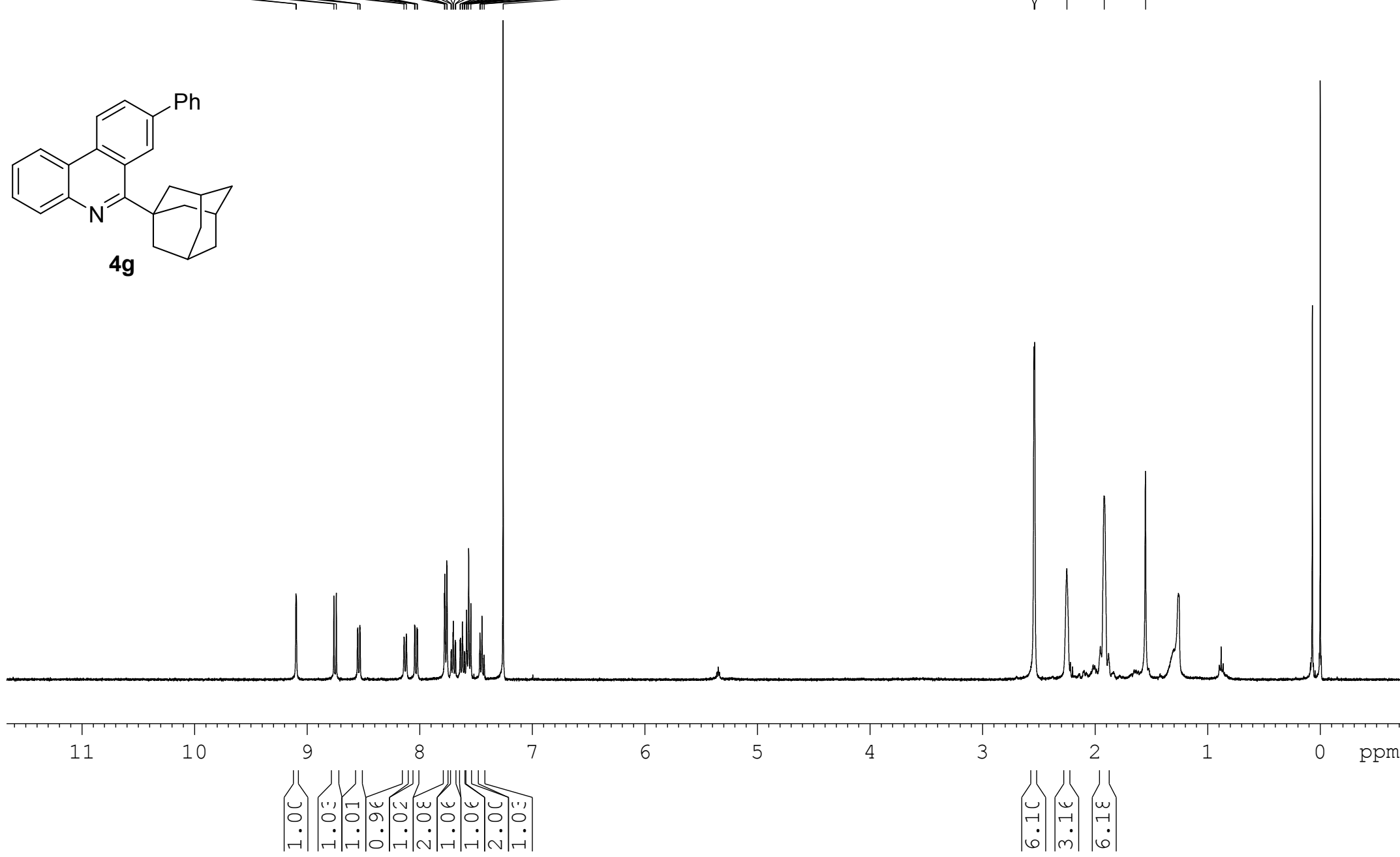

Figure S17. ${ }^{1} \mathrm{H}$ NMR spectrum of $\mathbf{4 g}\left(\mathrm{CDCl}_{3}, 400 \mathrm{MHz}\right)$. 

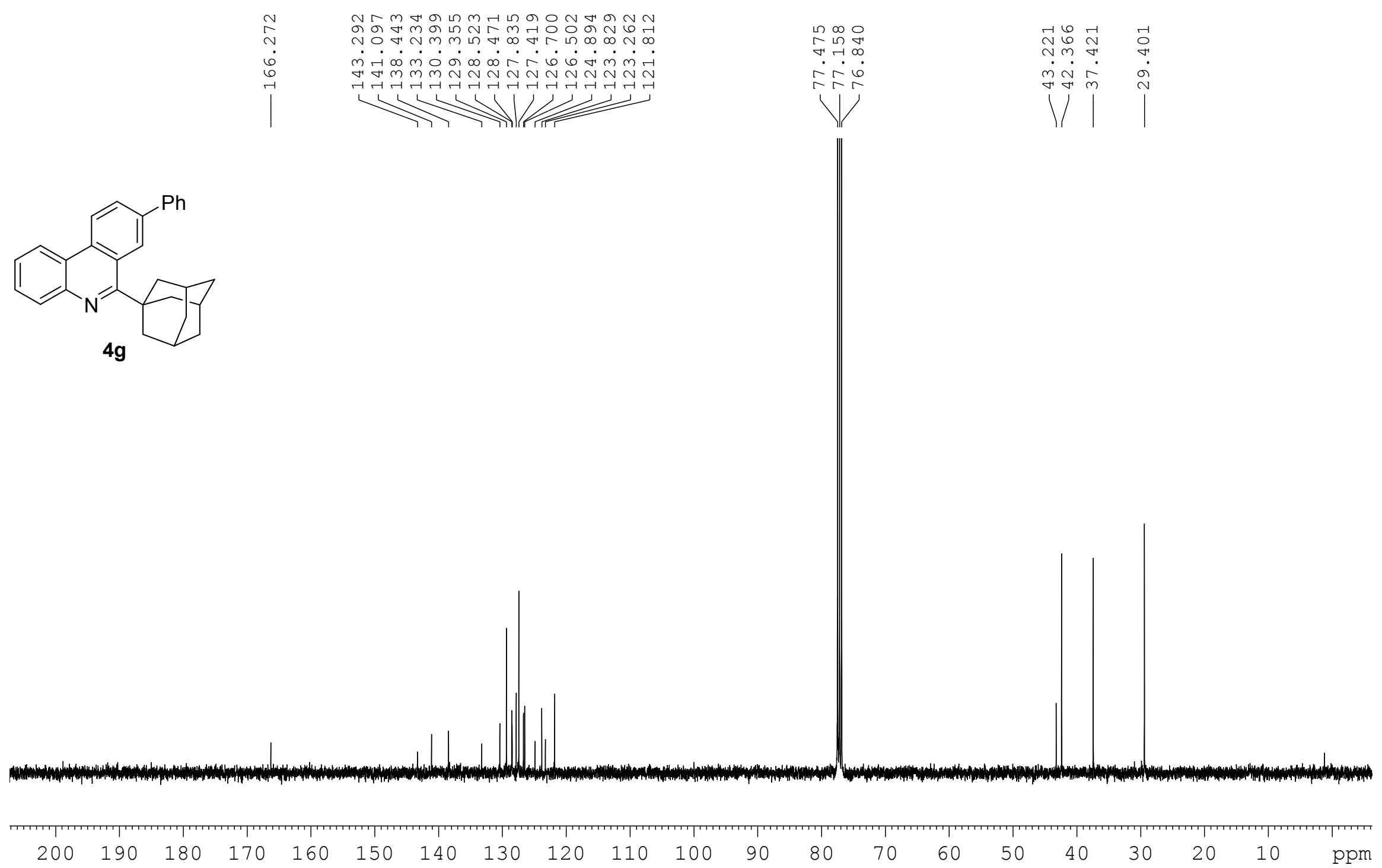

Figure S18. ${ }^{13} \mathrm{C}$ NMR spectrum of $4 \mathrm{~g}\left(\mathrm{CDCl}_{3}, 100 \mathrm{MHz}\right)$. 


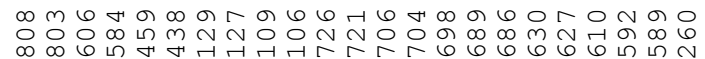

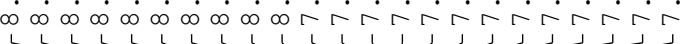
$\longrightarrow$

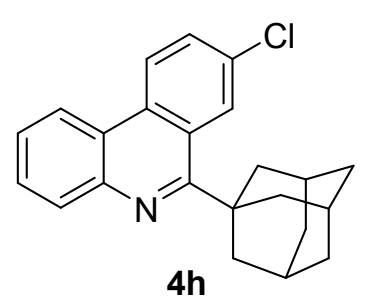

$4 h$

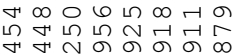

vivivi

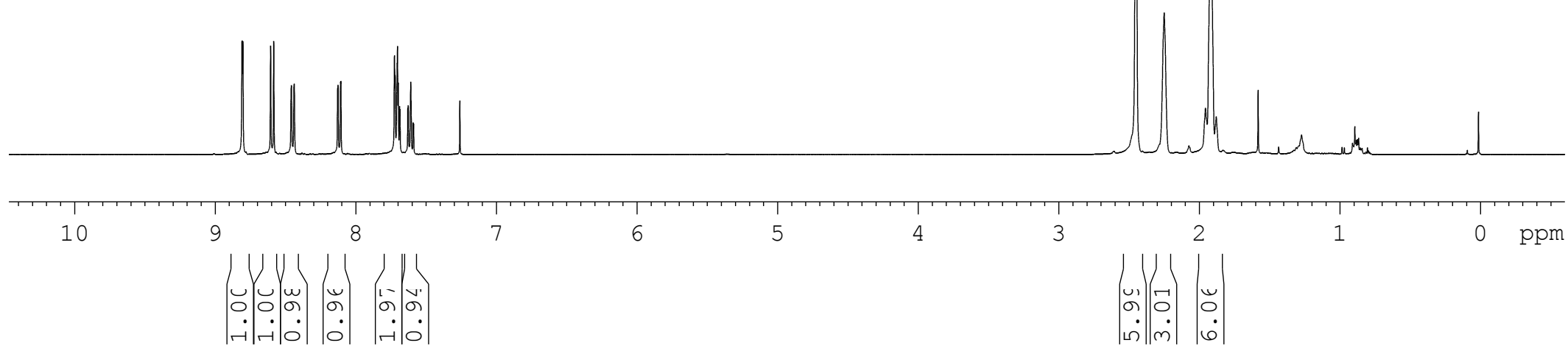


Figure S19. ${ }^{1} \mathrm{H}$ NMR spectrum of $\mathbf{4 h}\left(\mathrm{CDCl}_{3}, 400 \mathrm{MHz}\right)$.
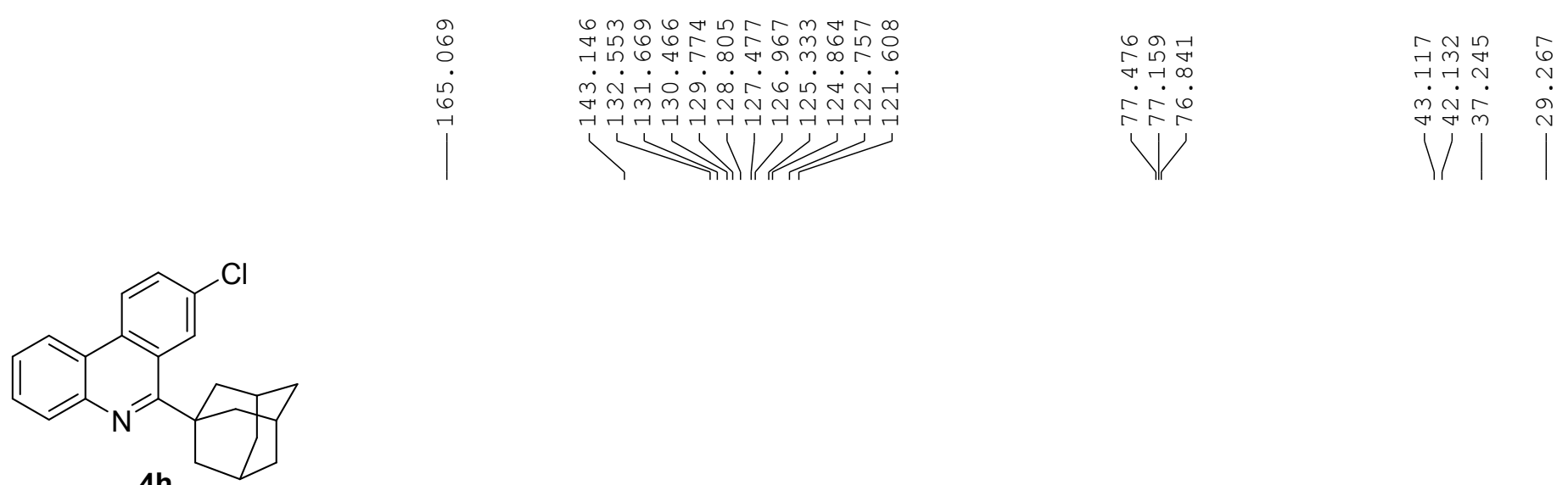

$4 h$

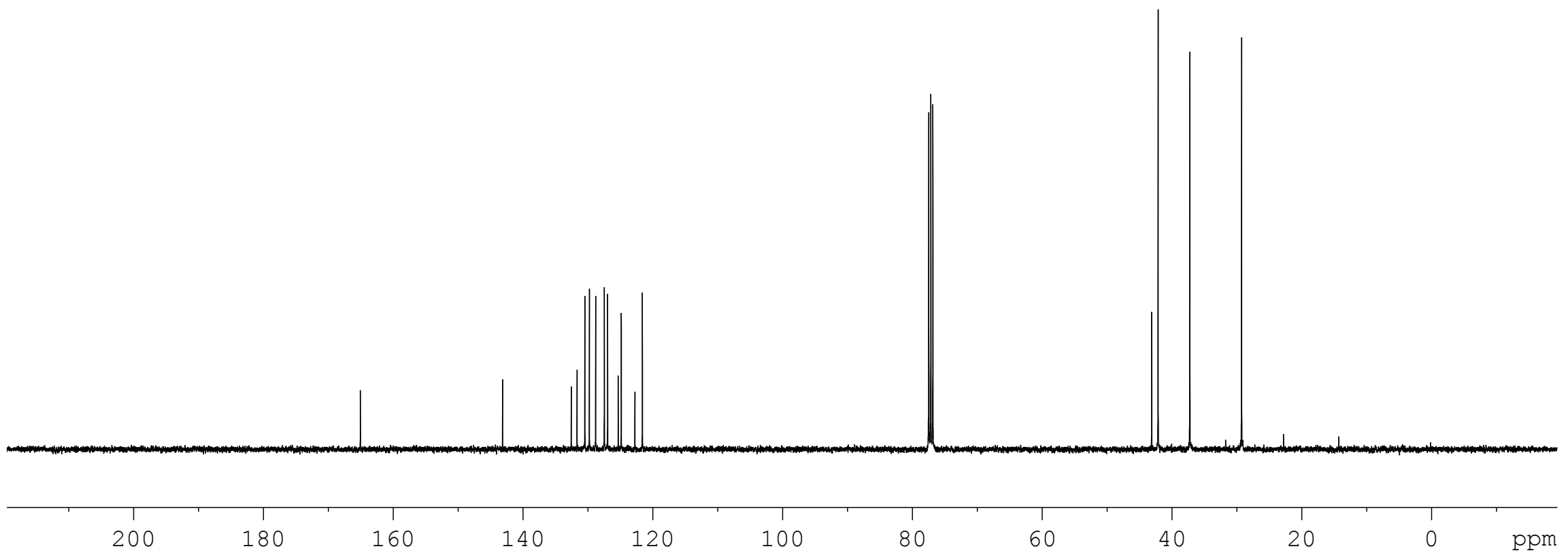

Figure S20. ${ }^{13} \mathrm{C}$ NMR spectrum of $4 \mathbf{h}\left(\mathrm{CDCl}_{3}, 100 \mathrm{MHz}\right)$ 


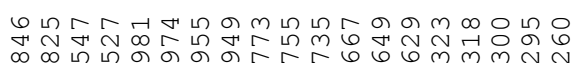

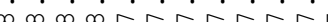

$>_{\infty}^{\infty}$

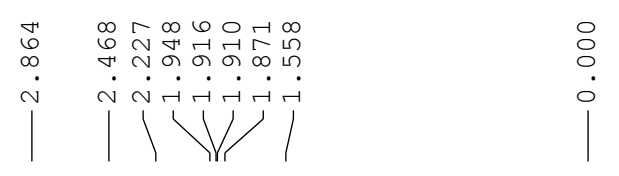

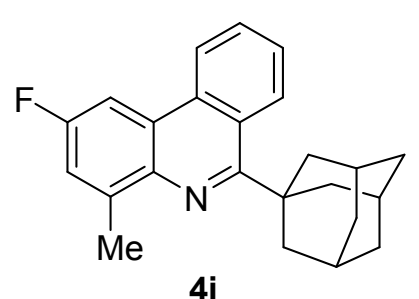

$$
\text { H }
$$

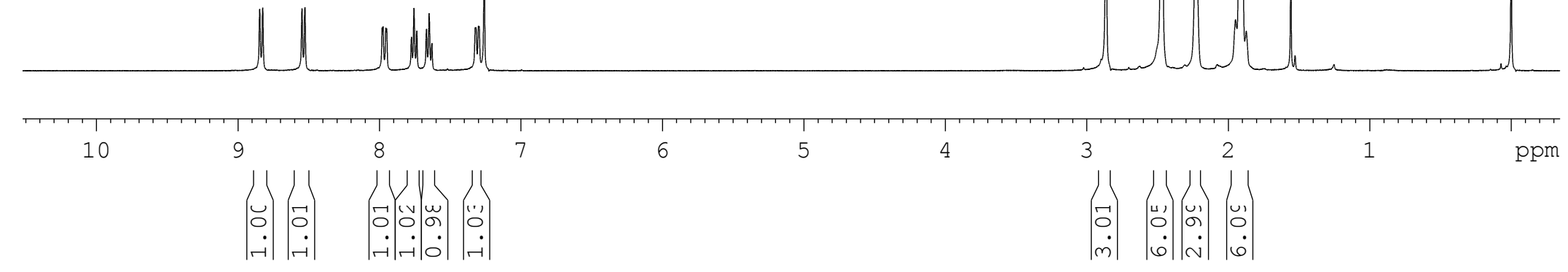


Figure S21. ${ }^{1} \mathrm{H}$ NMR spectrum of $\mathbf{4 i}\left(\mathrm{CDCl}_{3}, 400 \mathrm{MHz}\right)$.

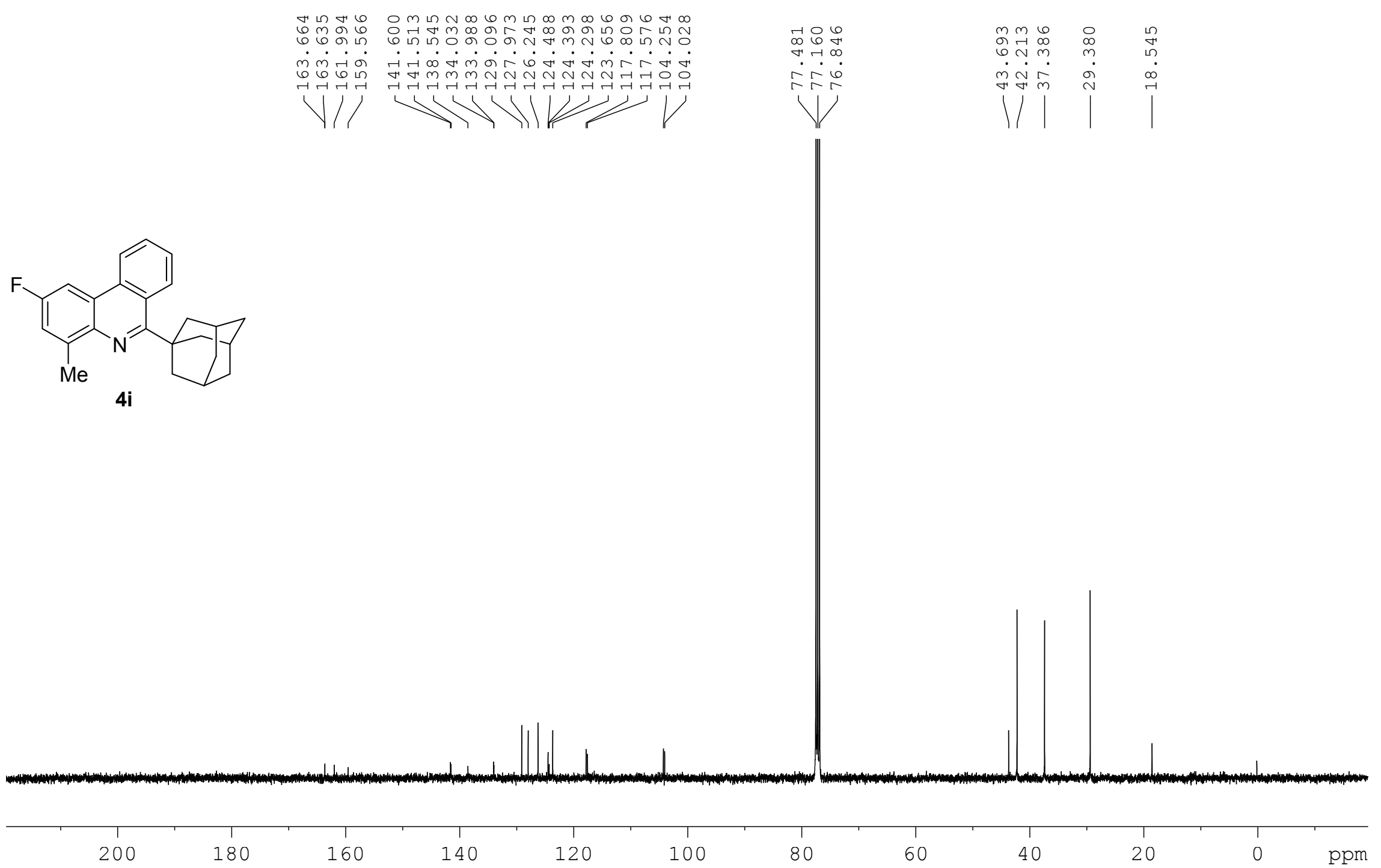

Figure S22. ${ }^{13} \mathrm{C}$ NMR spectrum of $4 \mathbf{i}\left(\mathrm{CDCl}_{3}, 100 \mathrm{MHz}\right)$. 


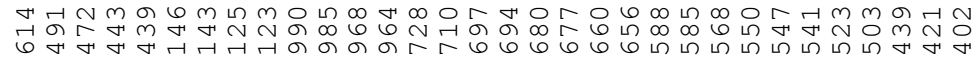
1
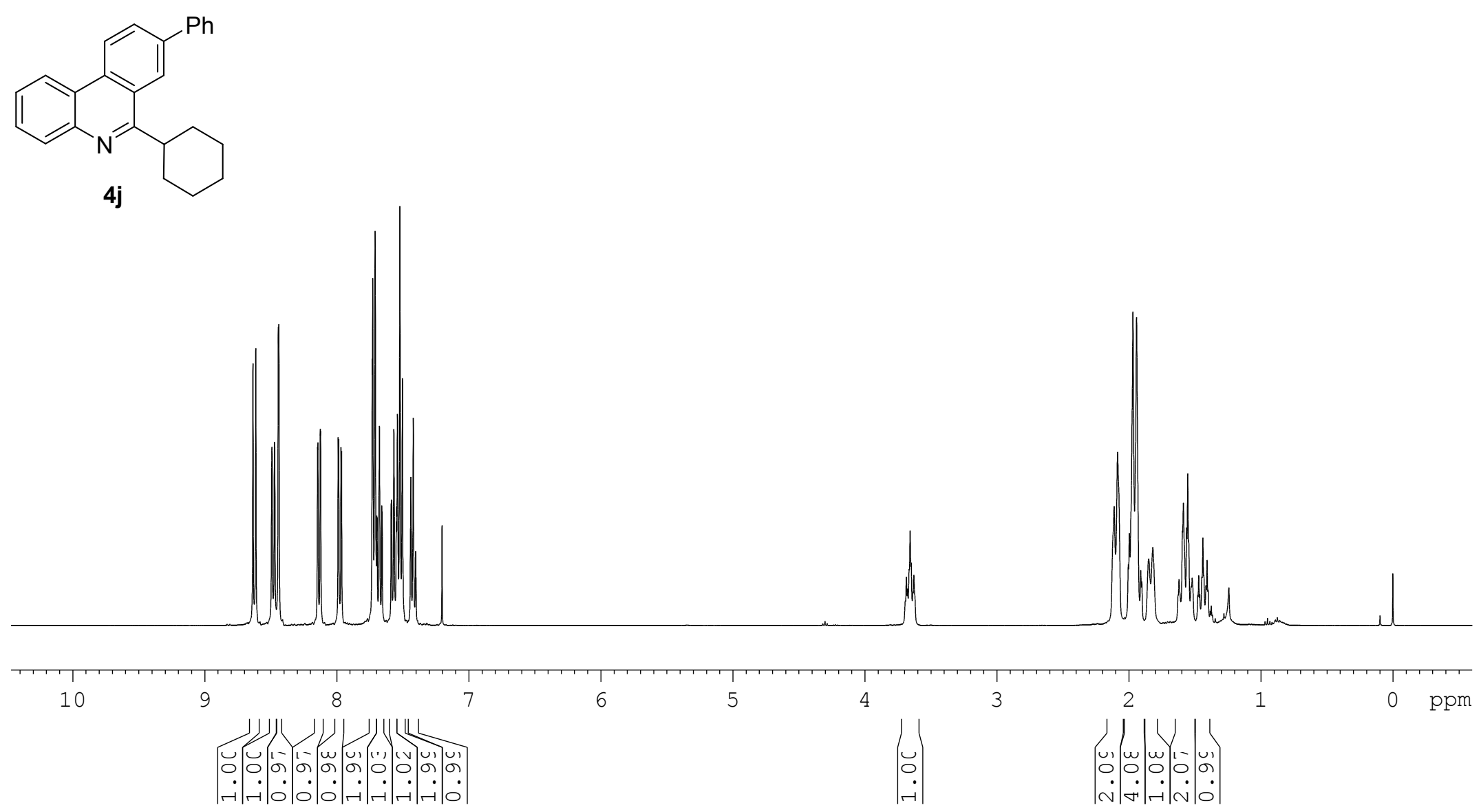
Figure S23. ${ }^{1} \mathrm{H}$ NMR spectrum of $\mathbf{4 j}\left(\mathrm{CDCl}_{3}, 400 \mathrm{MHz}\right)$.
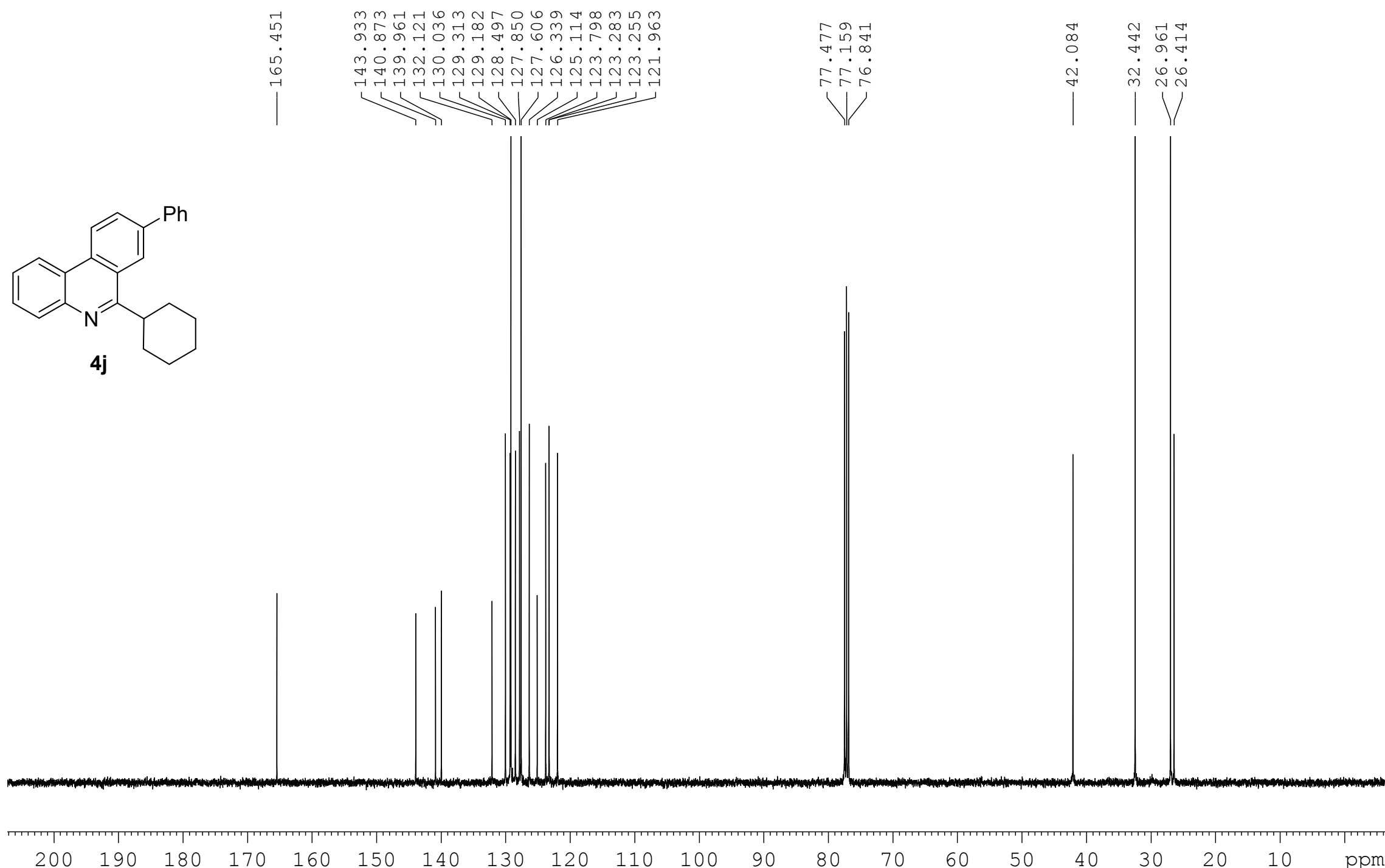

Figure S24. ${ }^{13} \mathrm{C}$ NMR spectrum of $\mathbf{4 j}\left(\mathrm{CDCl}_{3}, 100 \mathrm{MHz}\right)$. 

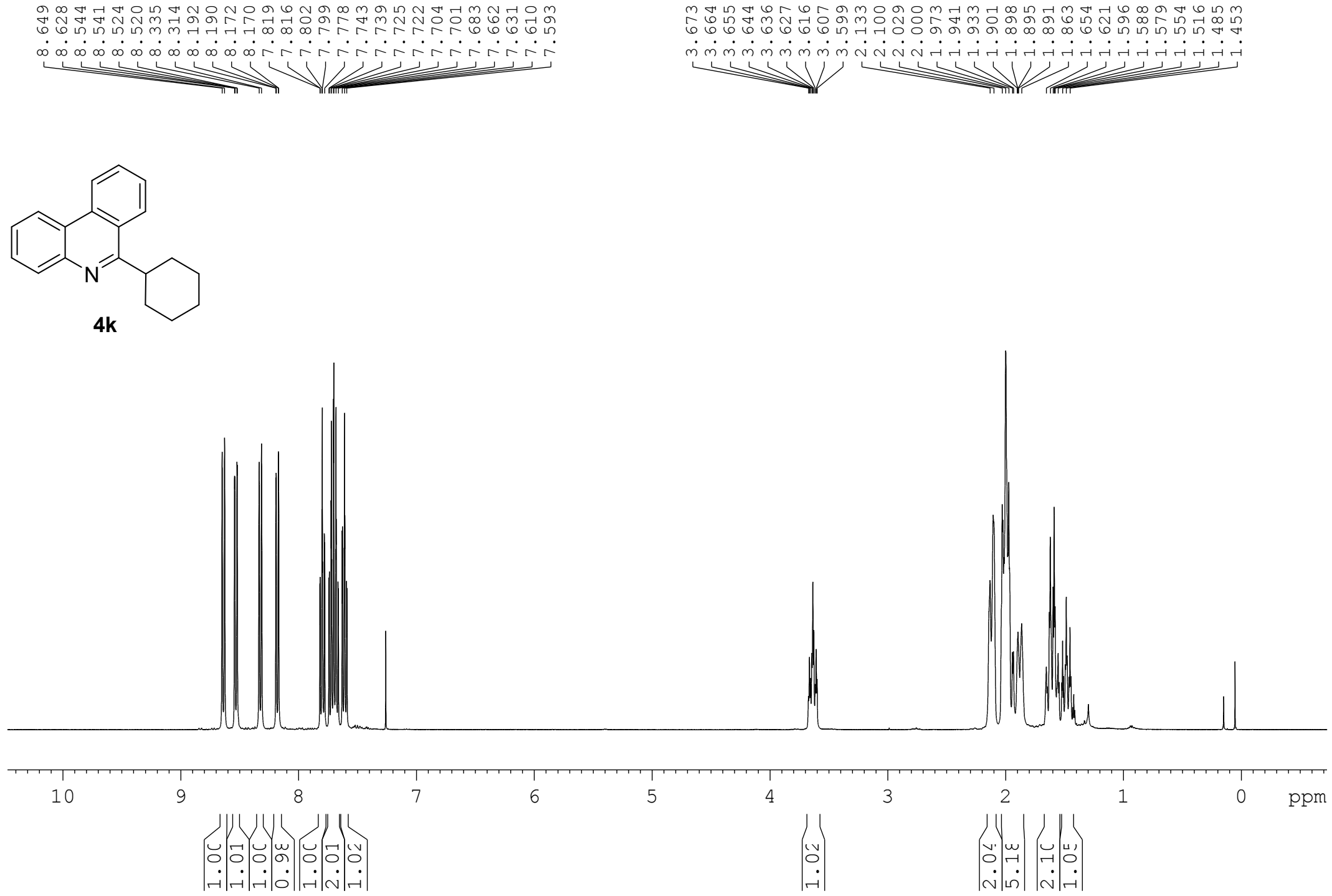

Figure S25. ${ }^{1} \mathrm{H}$ NMR spectrum of $\mathbf{4 k}\left(\mathrm{CDCl}_{3}, 400 \mathrm{MHz}\right)$.

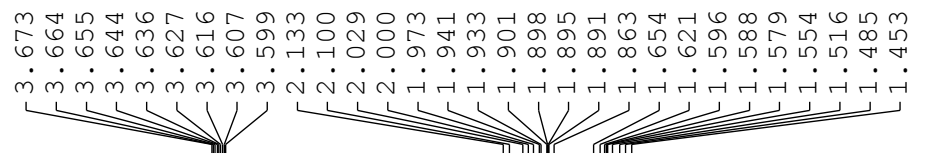

S28 


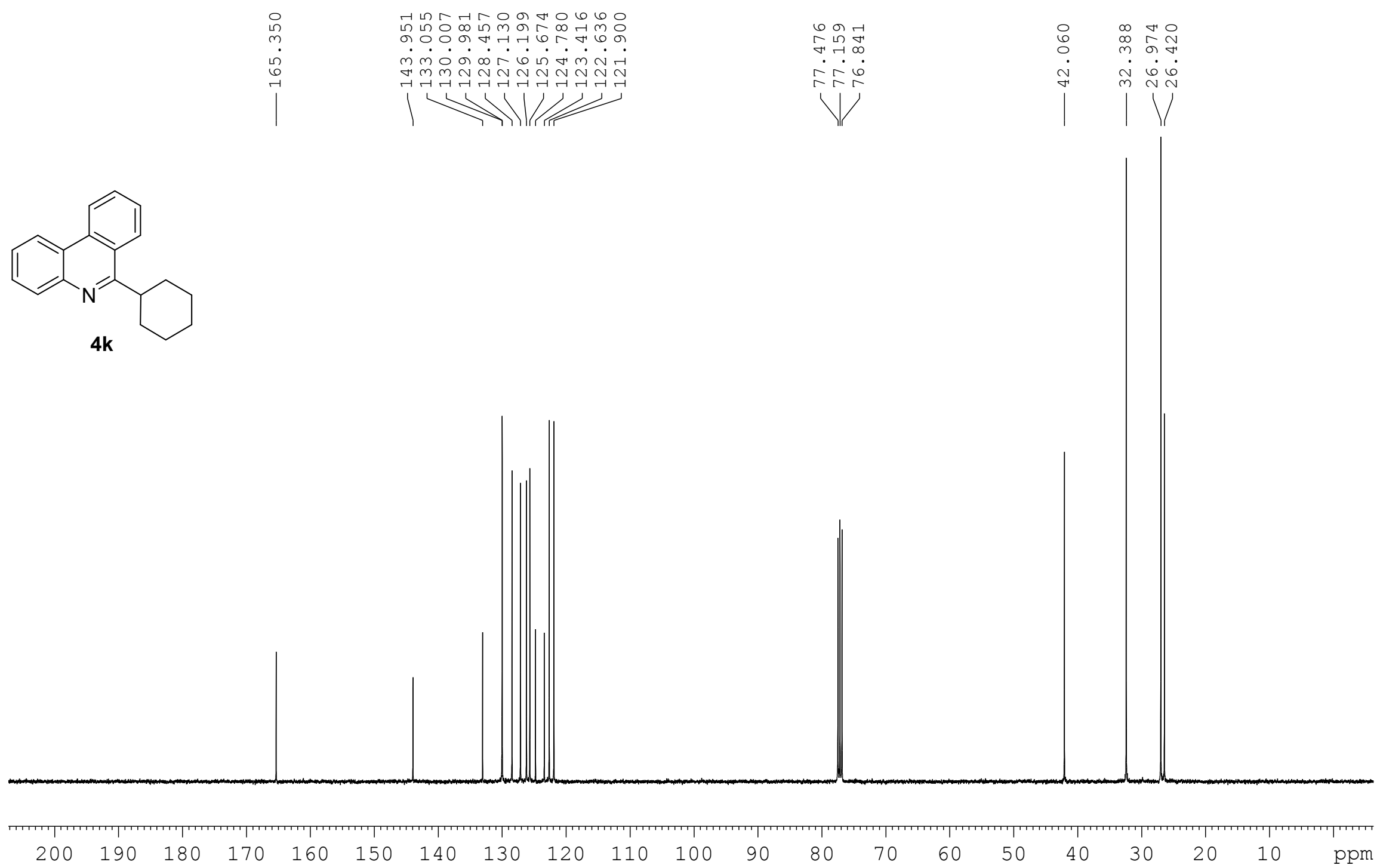

Figure S26. ${ }^{13} \mathrm{C}$ NMR spectrum of $\mathbf{4 k}\left(\mathrm{CDCl}_{3}, 100 \mathrm{MHz}\right)$. 


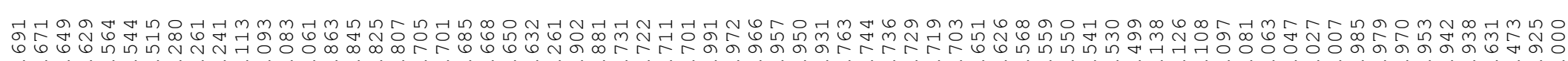

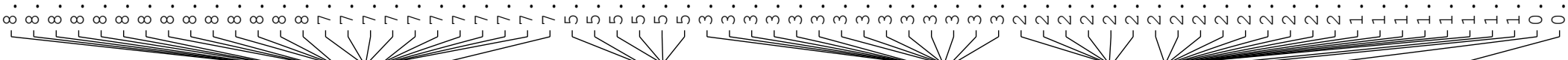

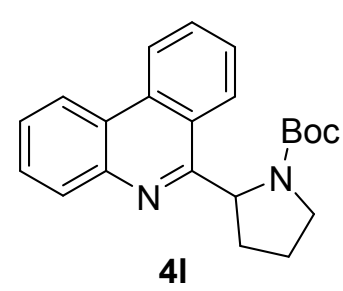

Boc

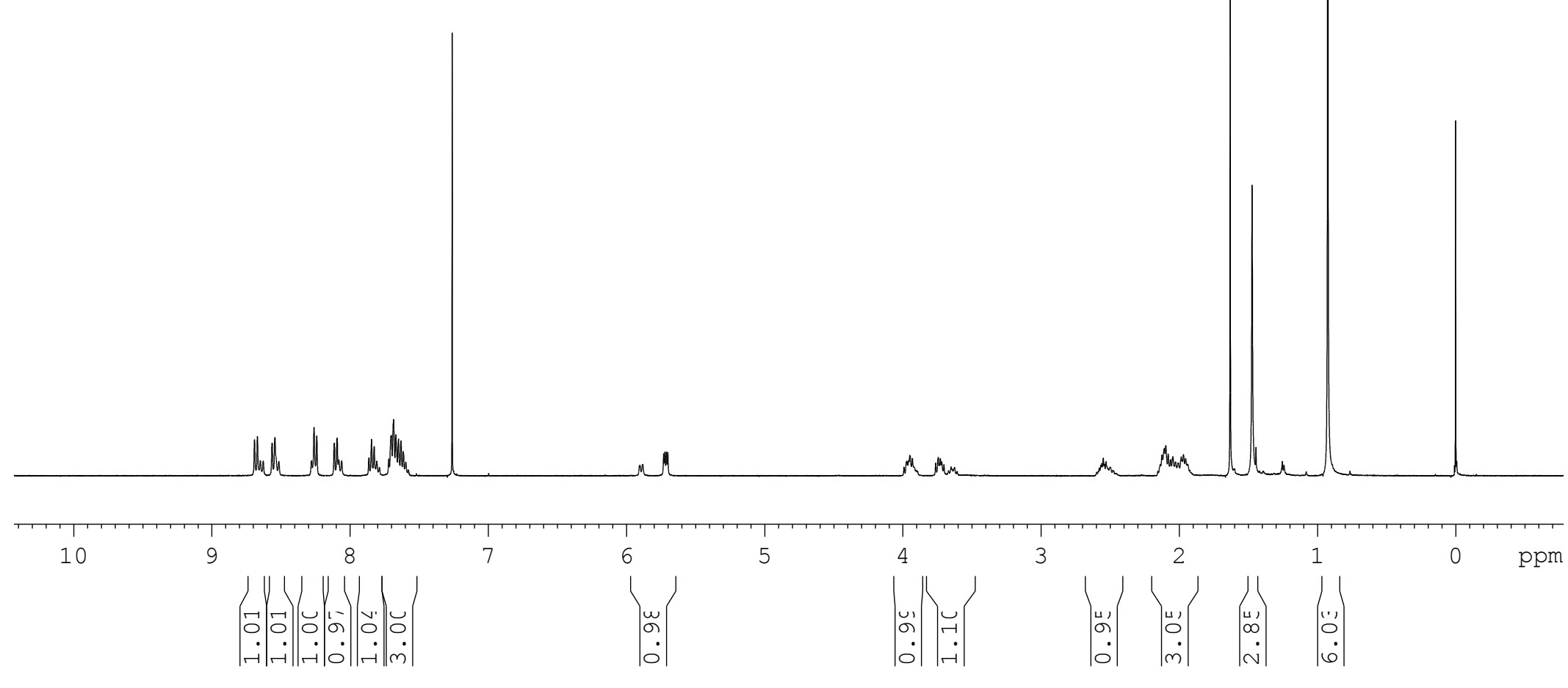


Figure S27. ${ }^{1} \mathrm{H}$ NMR spectrum of $\mathbf{4 l}\left(\mathrm{CDCl}_{3}, 400 \mathrm{MHz}\right)$. 

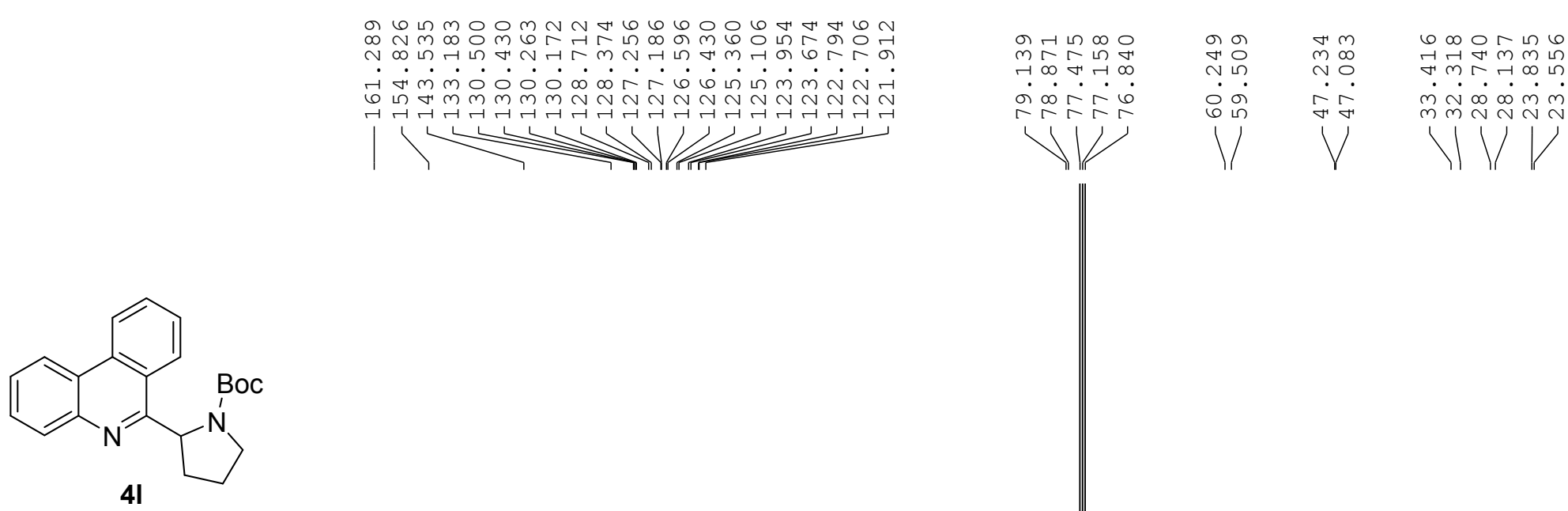

4

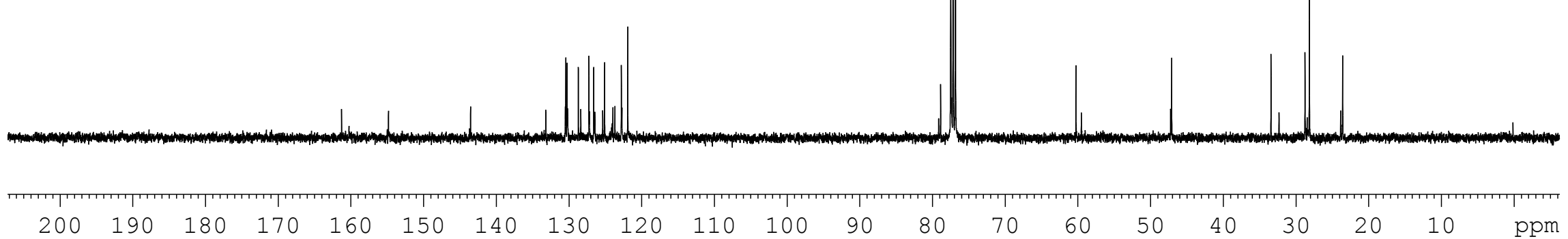

Figure S28. ${ }^{13} \mathrm{C}$ NMR spectrum of $4 \mathbf{l}\left(\mathrm{CDCl}_{3}, 100 \mathrm{MHz}\right)$. 


$$
\begin{aligned}
& \text { 곡욕염 } \\
& \text { ज्ञात क्ष } \\
& \text { VIV }
\end{aligned}
$$
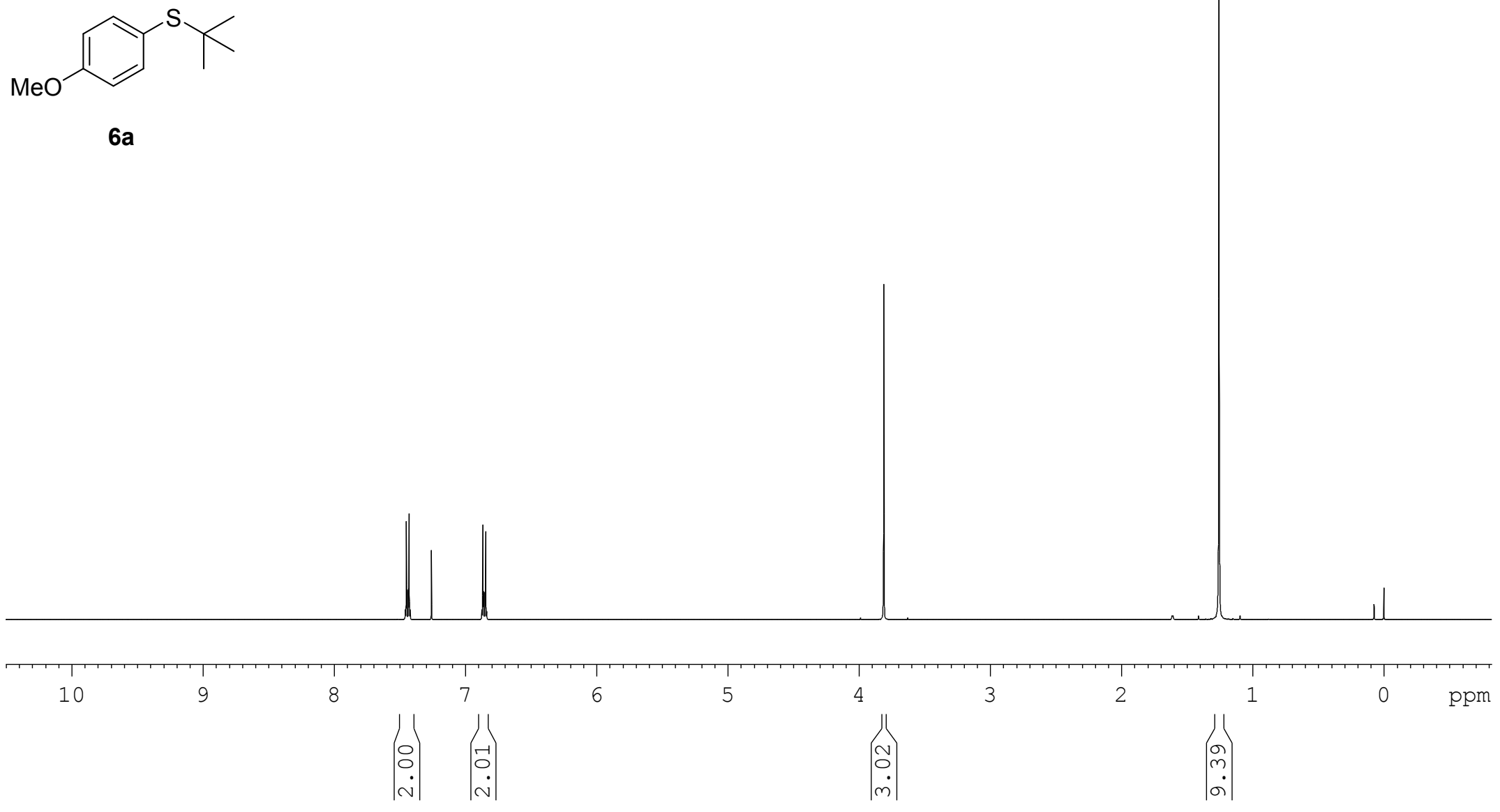

Figure S29. ${ }^{1} \mathrm{H}$ NMR spectrum of $6 \mathbf{6 a}\left(\mathrm{CDCl}_{3}, 400 \mathrm{MHz}\right)$. 


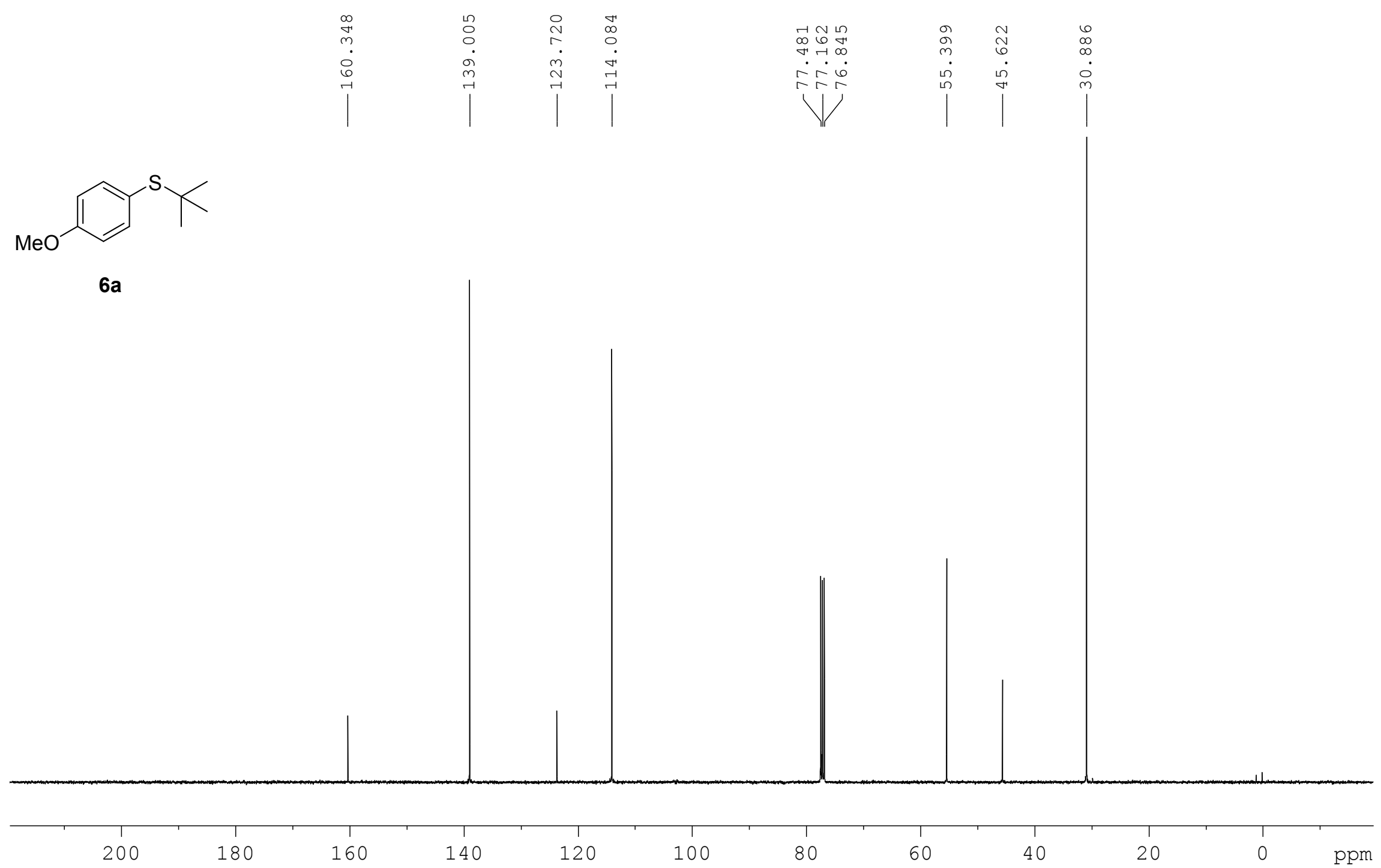

Figure S30. ${ }^{13} \mathrm{C}$ NMR spectrum of $\mathbf{6 a}\left(\mathrm{CDCl}_{3}, 100 \mathrm{MHz}\right)$. 


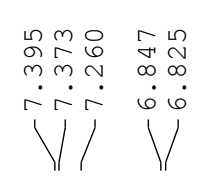
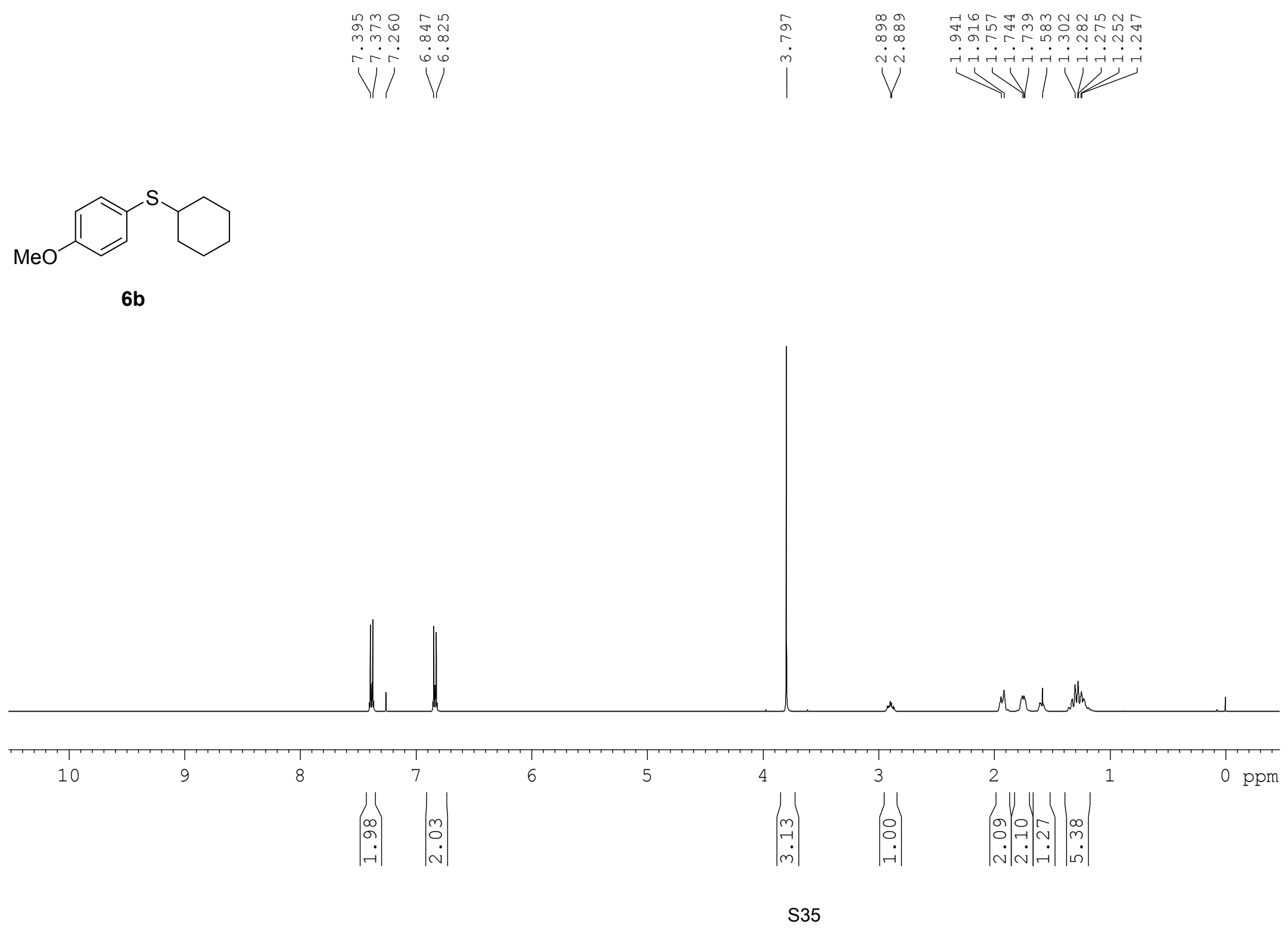
Figure S31. ${ }^{1} \mathrm{H}$ NMR spectrum of $\mathbf{6 b}\left(\mathrm{CDCl}_{3}, 400 \mathrm{MHz}\right)$.
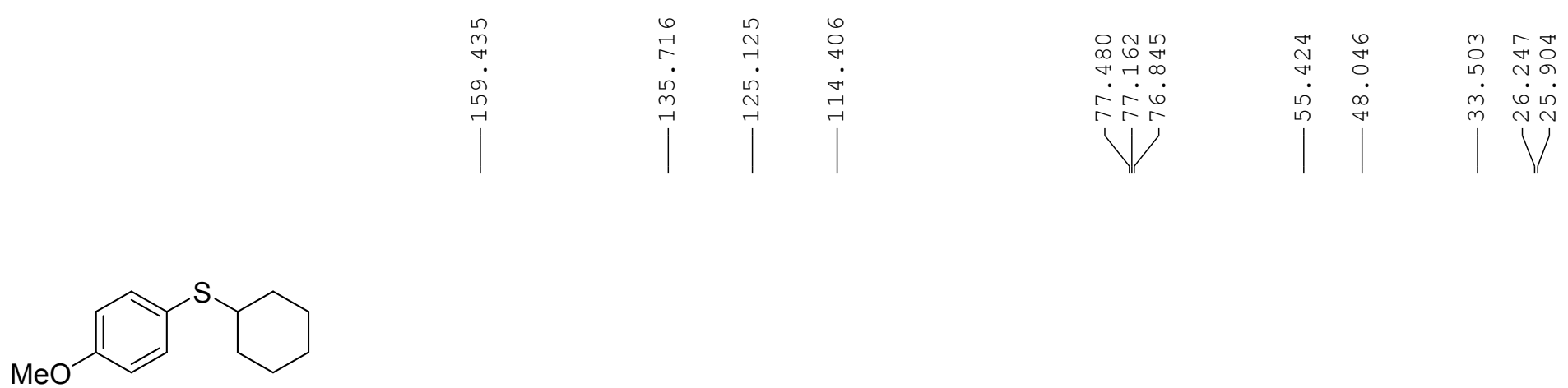

$6 \mathrm{~b}$

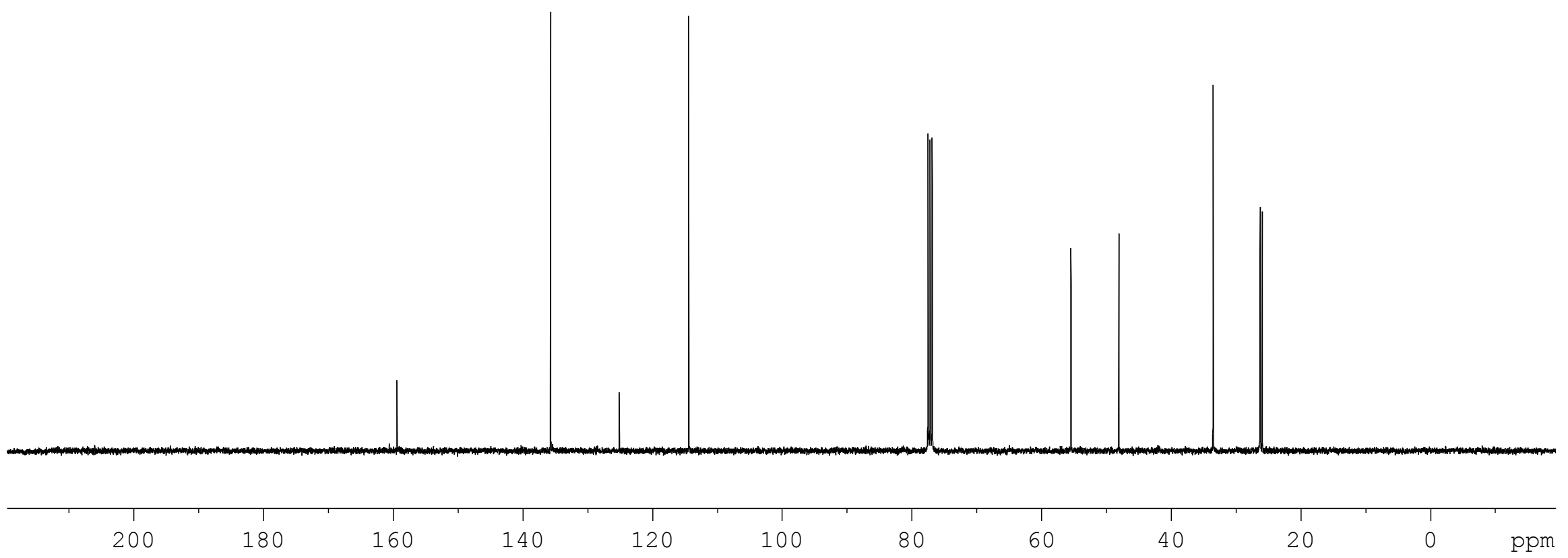

Figure S32. ${ }^{13} \mathrm{C}$ NMR spectrum of $6 \mathbf{b}\left(\mathrm{CDCl}_{3}, 100 \mathrm{MHz}\right)$. 

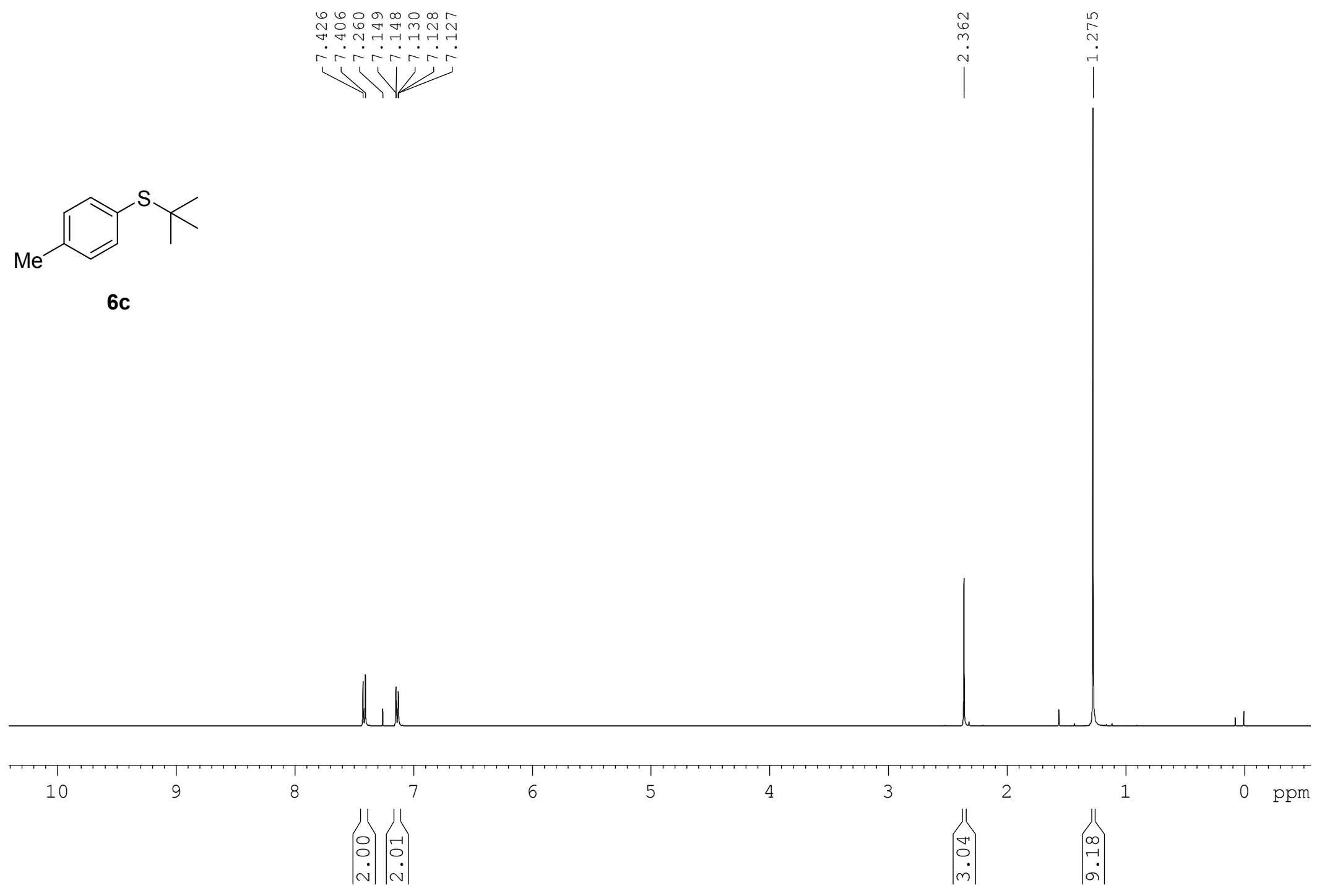

S37

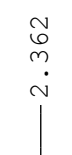

r

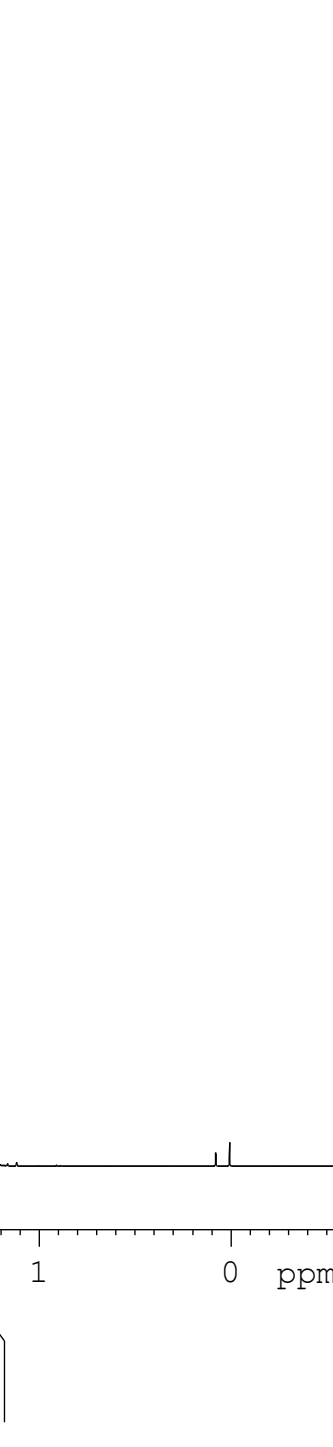


Figure S33. ${ }^{1} \mathrm{H}$ NMR spectrum of $\mathbf{6 c}\left(\mathrm{CDCl}_{3}, 400 \mathrm{MHz}\right)$
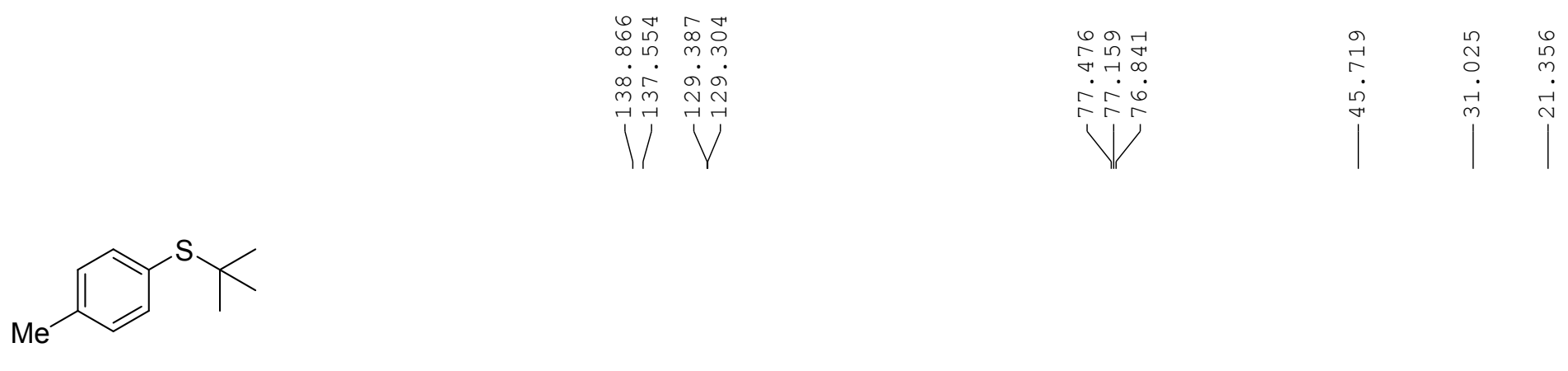

6c

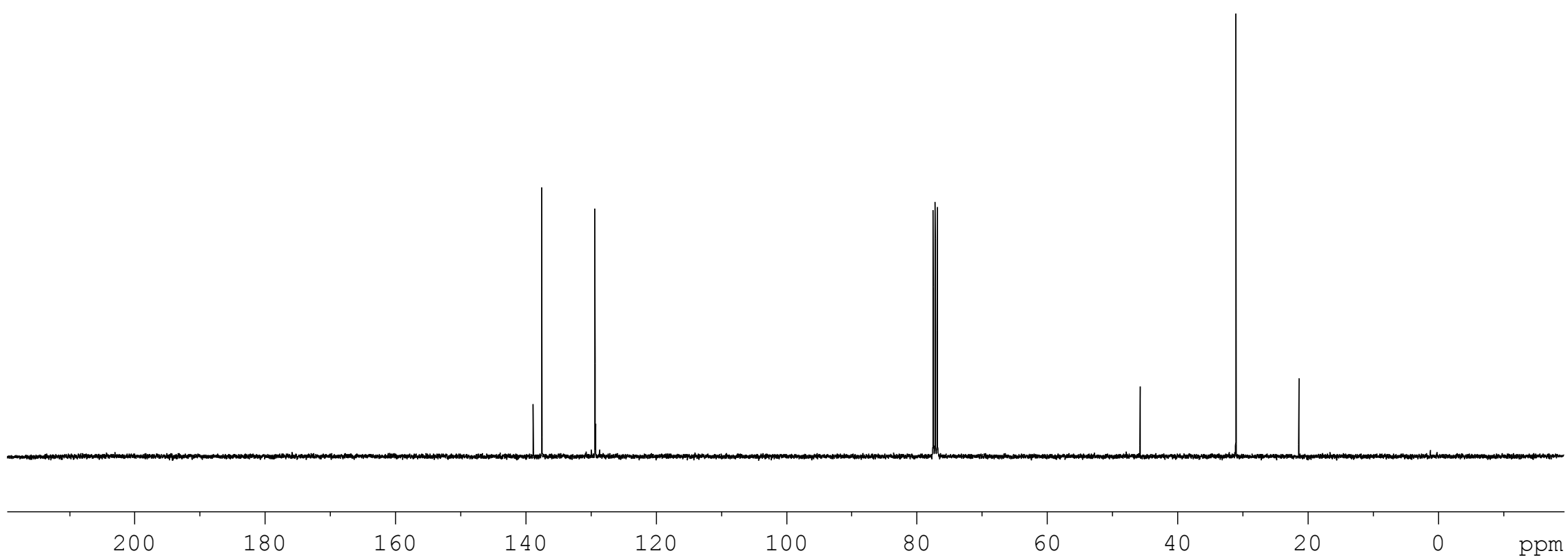

Figure S34. ${ }^{13} \mathrm{C}$ NMR spectrum of $6 \mathbf{c}\left(\mathrm{CDCl}_{3}, 100 \mathrm{MHz}\right)$. 

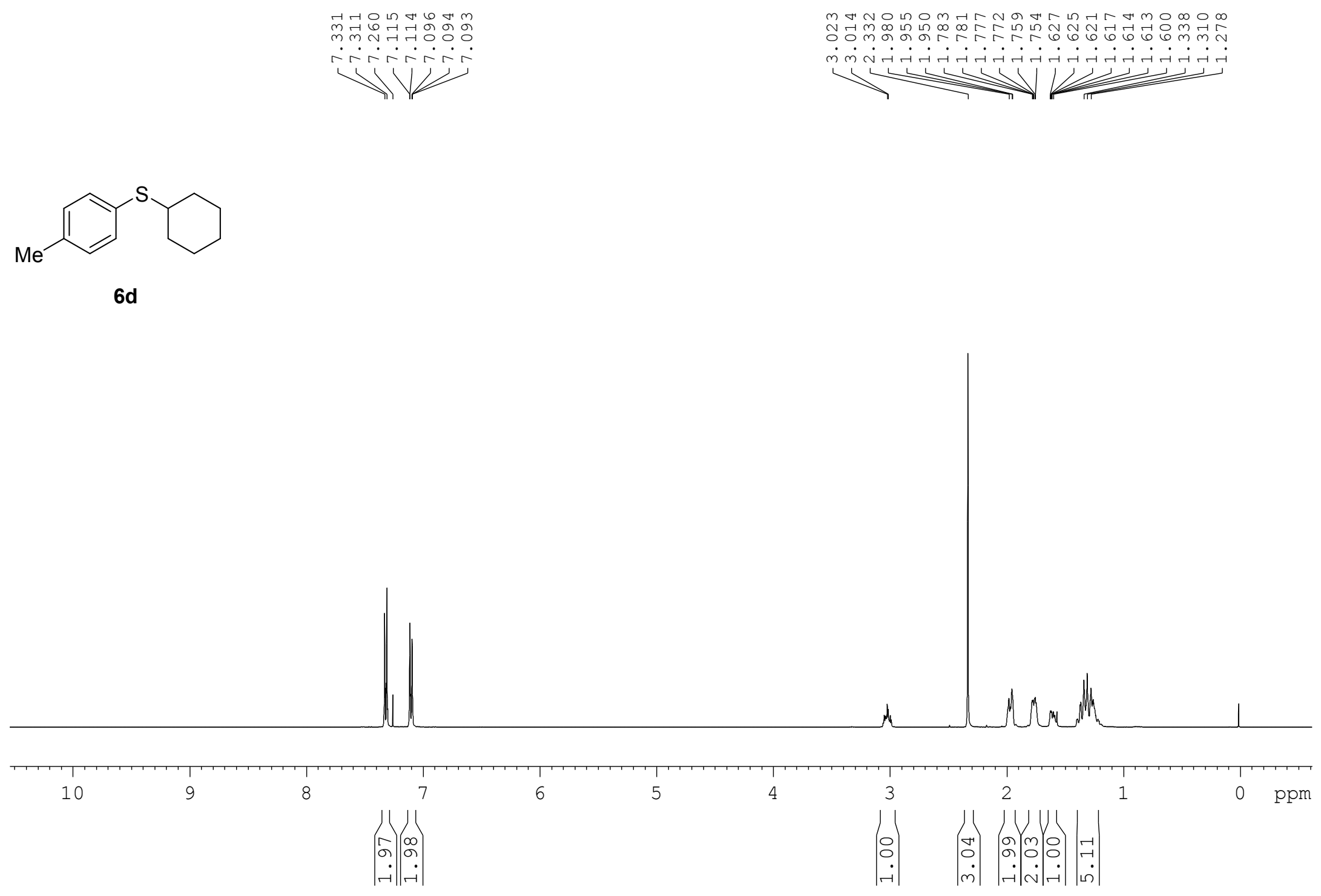
Figure S35. ${ }^{1} \mathrm{H}$ NMR spectrum of $\mathbf{6 d}\left(\mathrm{CDCl}_{3}, 400 \mathrm{MHz}\right)$.
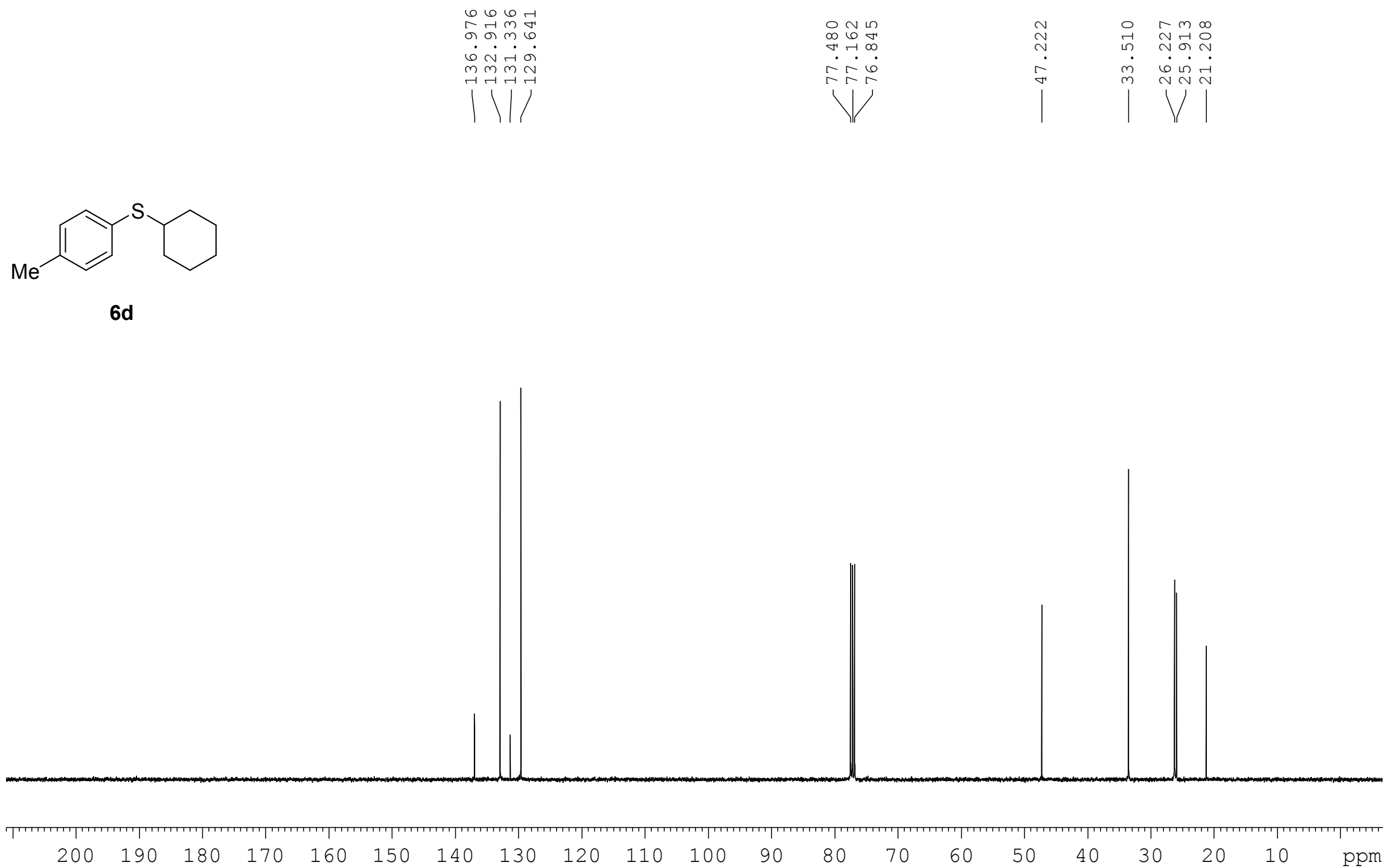

Figure S36. ${ }^{13} \mathrm{C}$ NMR spectrum of $\mathbf{6 d}\left(\mathrm{CDCl}_{3}, 100 \mathrm{MHz}\right)$ 


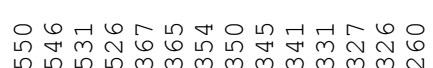

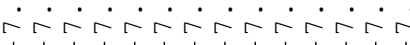

$\longrightarrow$

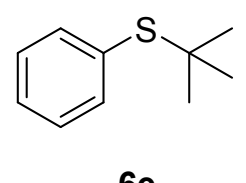

$6 e$

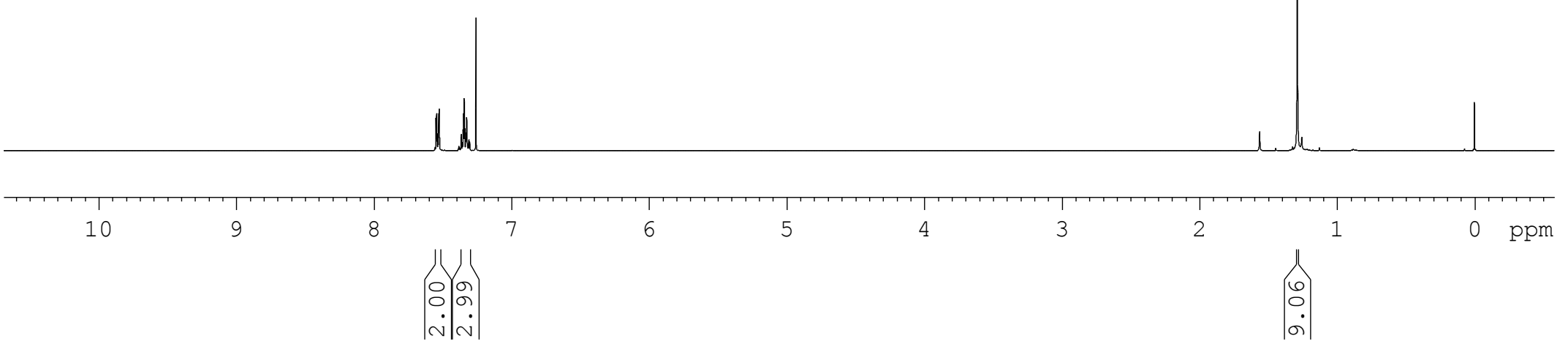




$$
\mathrm{all}
$$




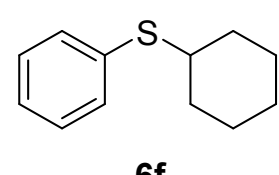

$6 f$

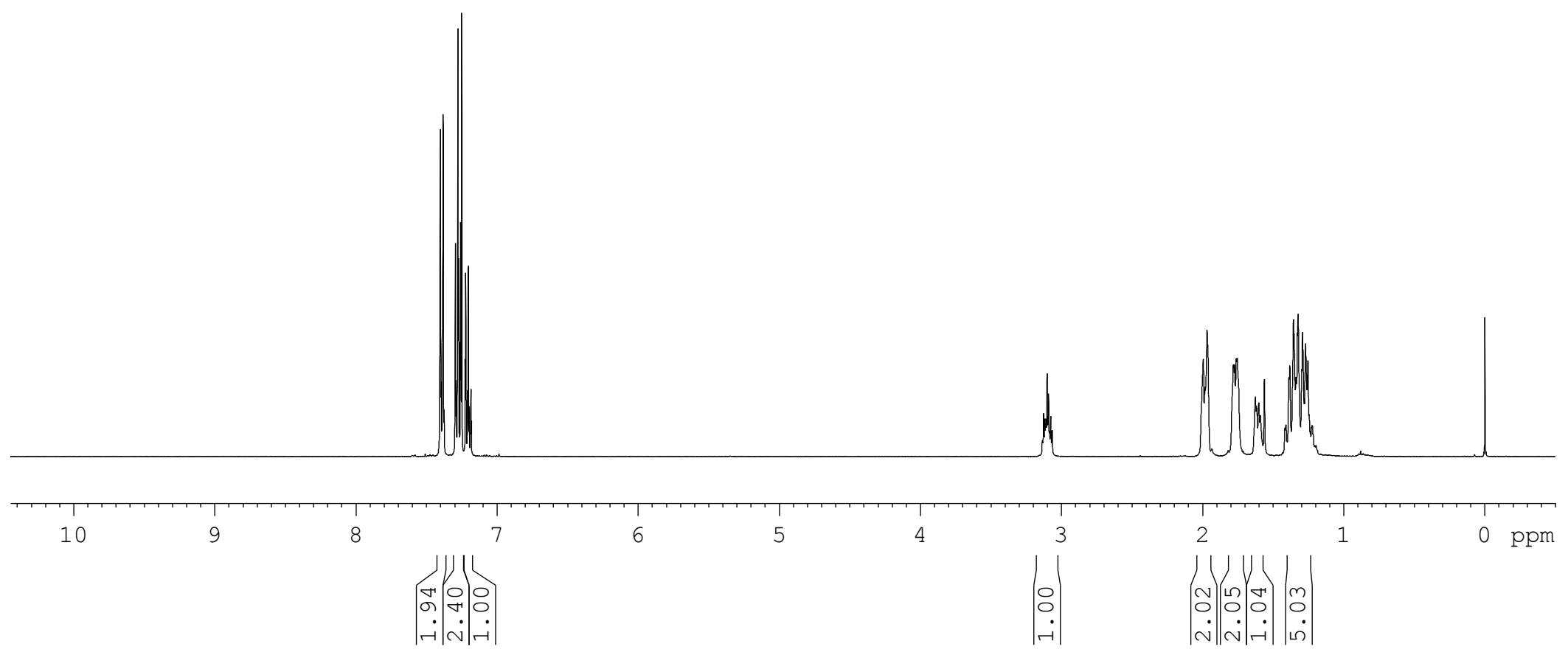

Figure S39. ${ }^{1} \mathrm{H}$ NMR spectrum of $\mathbf{6 f}\left(\mathrm{CDCl}_{3}, 400 \mathrm{MHz}\right)$. 


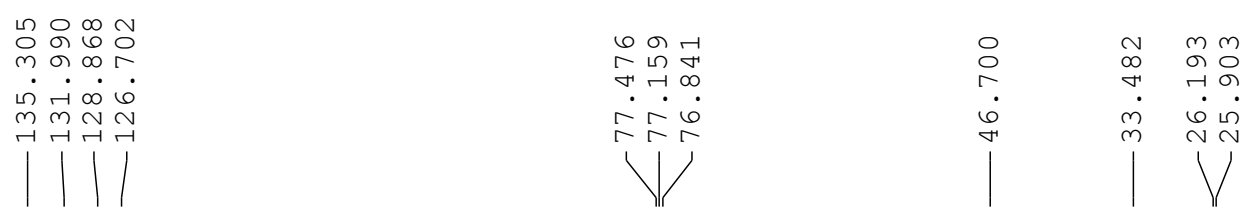

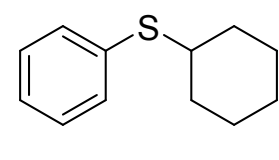

$6 f$

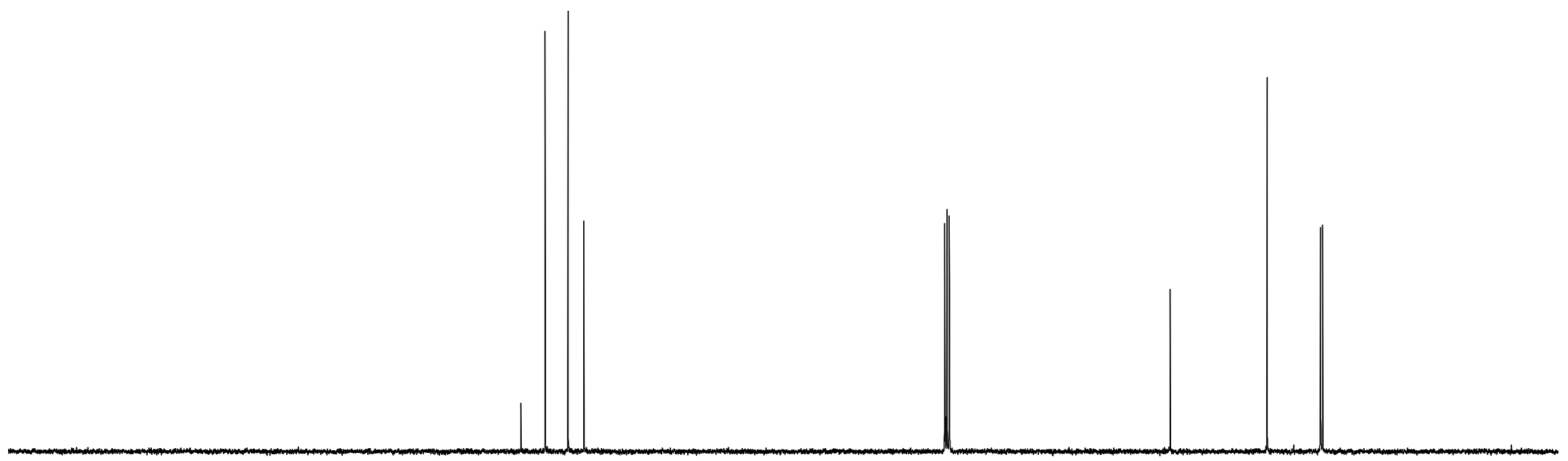

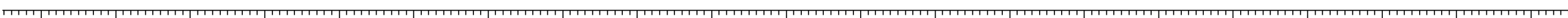

$\begin{array}{llllllllllllllllllllll}200 & 190 & 180 & 170 & 160 & 150 & 140 & 130 & 120 & 110 & 100 & 90 & 80 & 70 & 60 & 50 & 40 & 30 & 20 & 10 & \text { ppm }\end{array}$

Figure S40. ${ }^{13} \mathrm{C}$ NMR spectrum of $\mathbf{6 f}\left(\mathrm{CDCl}_{3}, 100 \mathrm{MHz}\right)$. 


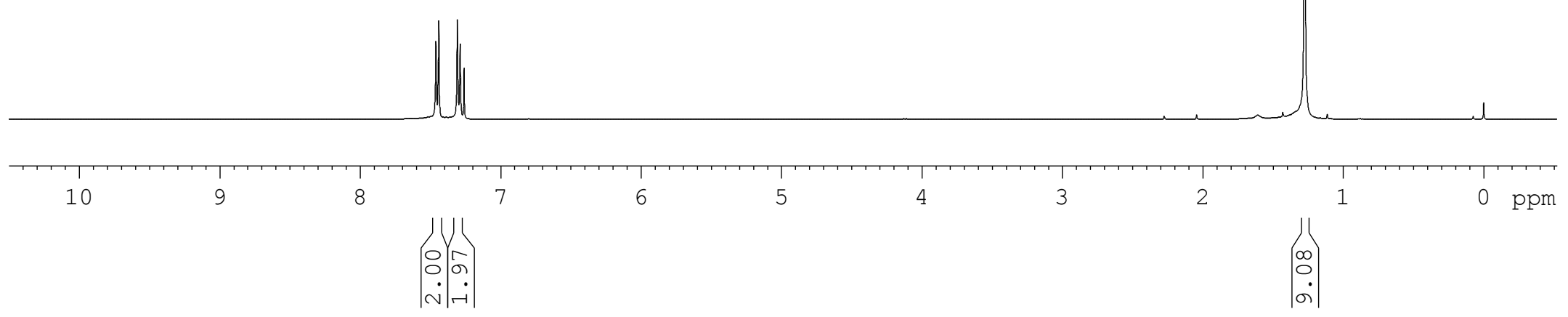

Figure S41. ${ }^{1} \mathrm{H}$ NMR spectrum of $\mathbf{6 g}\left(\mathrm{CDCl}_{3}, 400 \mathrm{MHz}\right)$. 

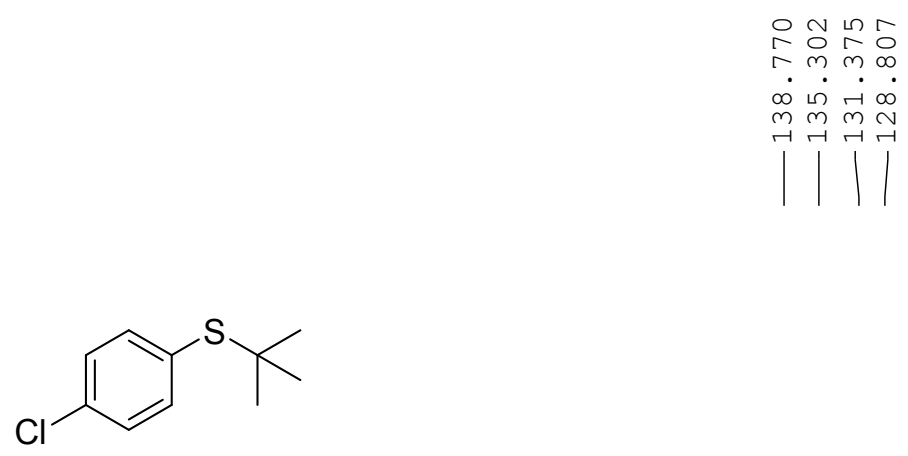

$6 \mathrm{~g}$

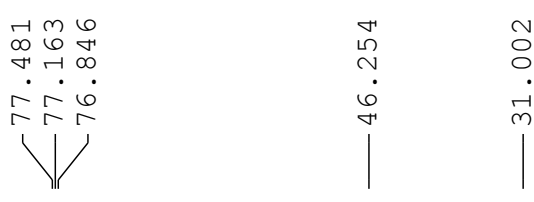

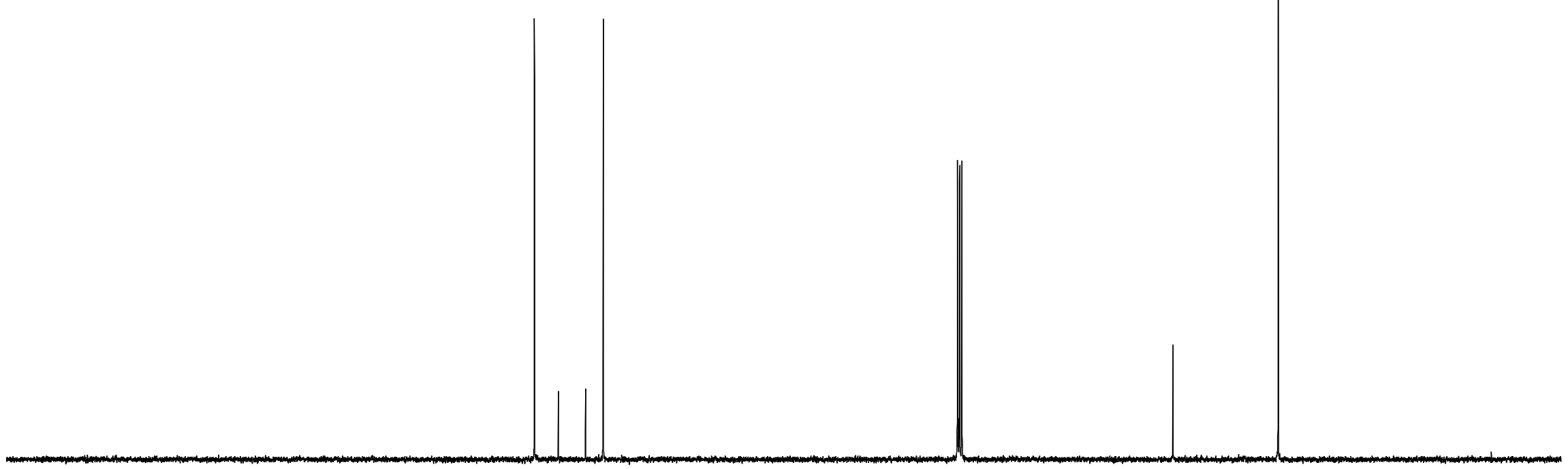

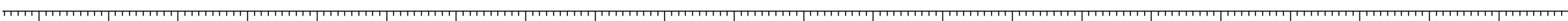

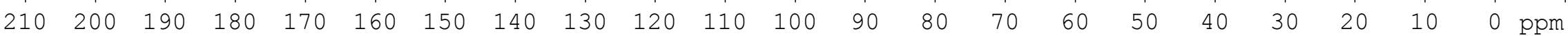

Figure S42. ${ }^{13} \mathrm{C}$ NMR spectrum of $\mathbf{6 g}\left(\mathrm{CDCl}_{3}, 100 \mathrm{MHz}\right)$ 


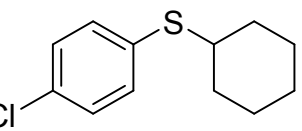

$6 h$

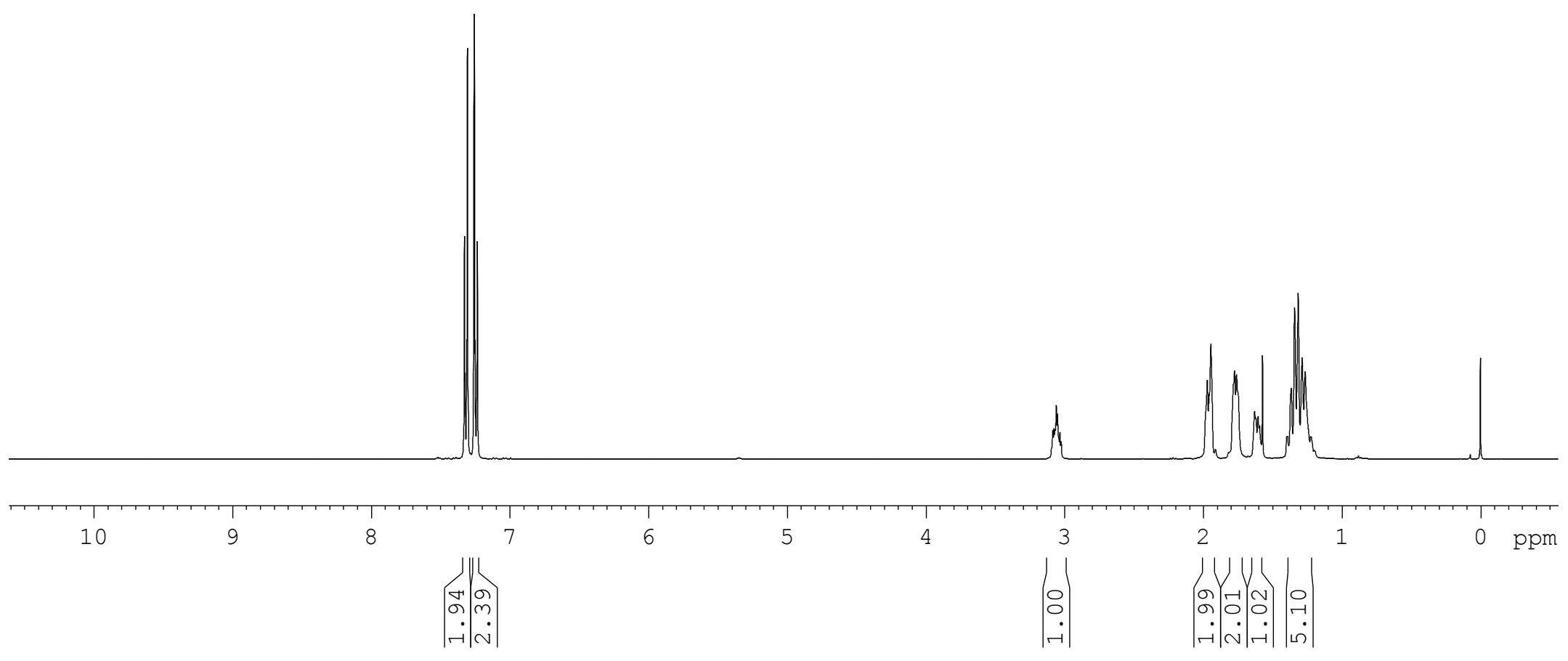

Figure S43. ${ }^{1} \mathrm{H}$ NMR spectrum of $\mathbf{6 i}\left(\mathrm{CDCl}_{3}, 400 \mathrm{MHz}\right)$ 


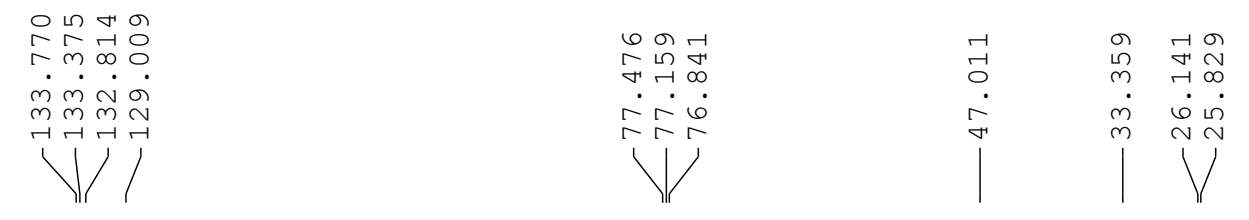

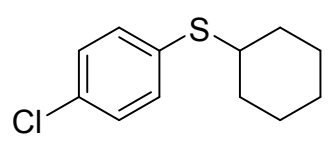

$6 h$

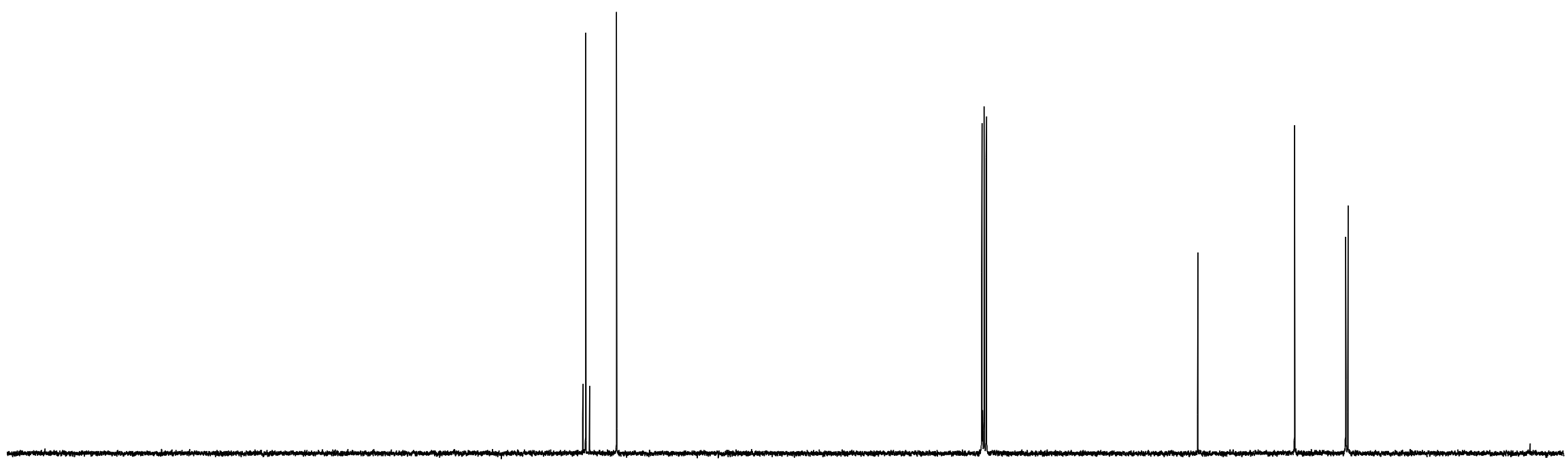
$210 \quad 20$
$80 \quad 17$
0140
13012
110100
$90 \quad 80$
$70 \quad 60$
50
302
10 ppm

Figure S44. ${ }^{13} \mathrm{C}$ NMR spectrum of $6 \mathbf{i}\left(\mathrm{CDCl}_{3}, 100 \mathrm{MHz}\right)$. 


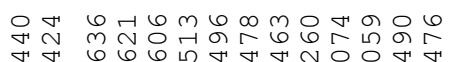

m

V vivif

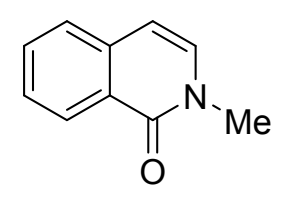

9a

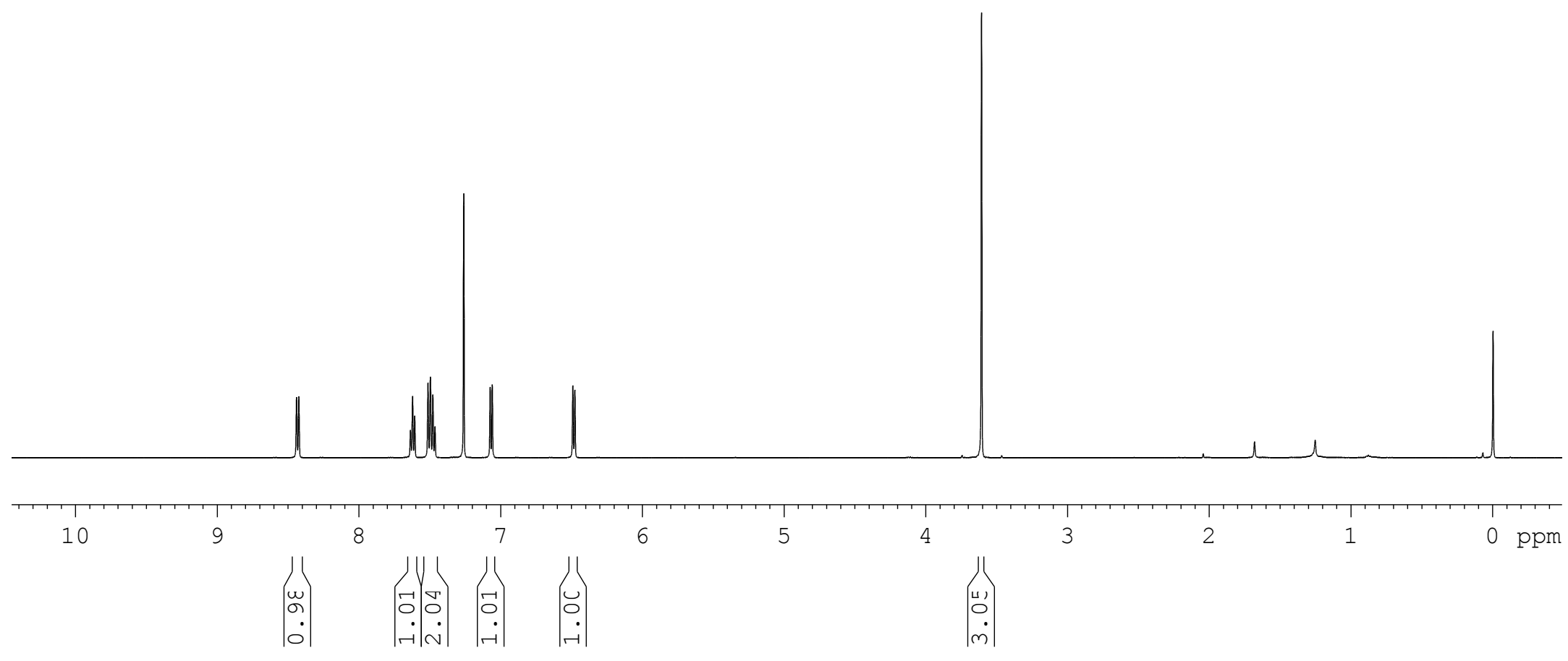

Figure S45. ${ }^{1} \mathrm{H}$ NMR spectrum of $9 a\left(\mathrm{CDCl}_{3}, 500 \mathrm{MHz}\right)$. 


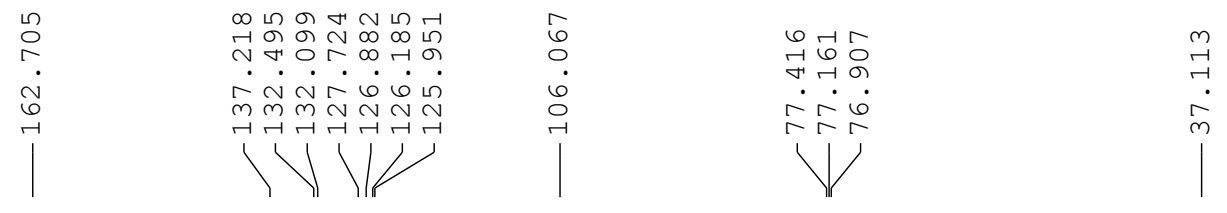

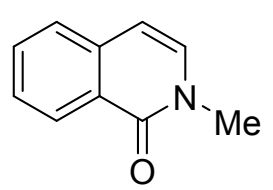

$9 a$

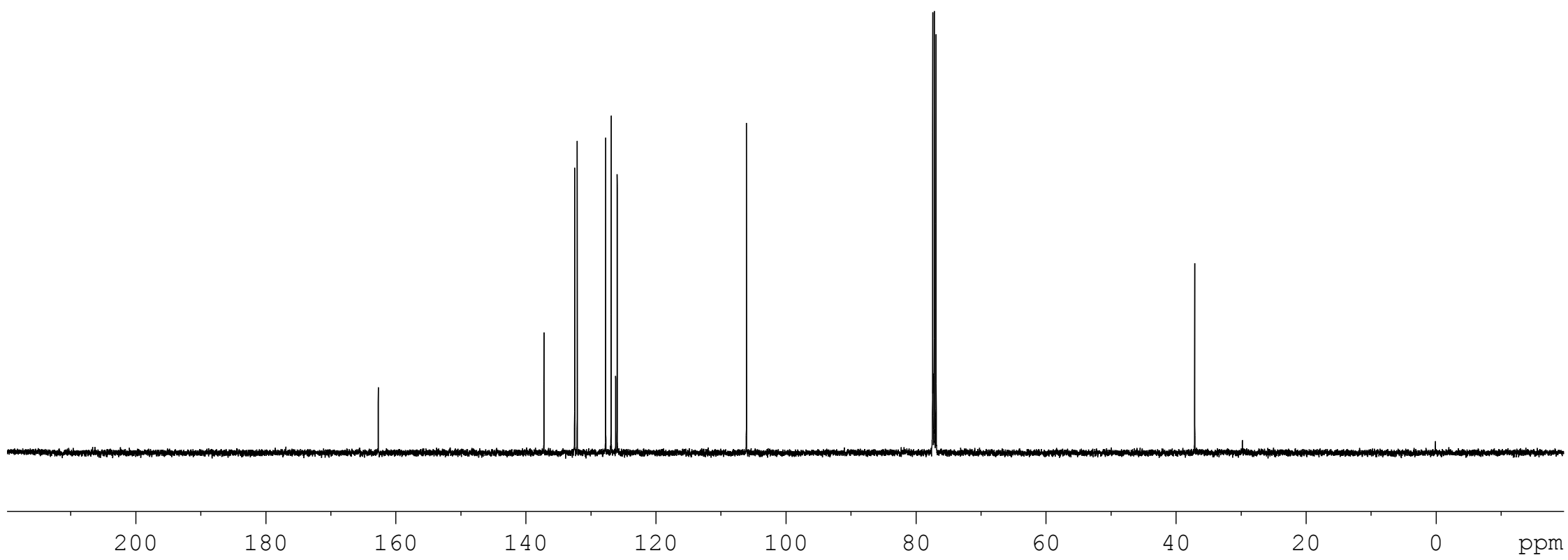

Figure S46. ${ }^{13} \mathrm{C}$ NMR spectrum of 9a $\left(\mathrm{CDCl}_{3}, 125 \mathrm{MHz}\right)$. 


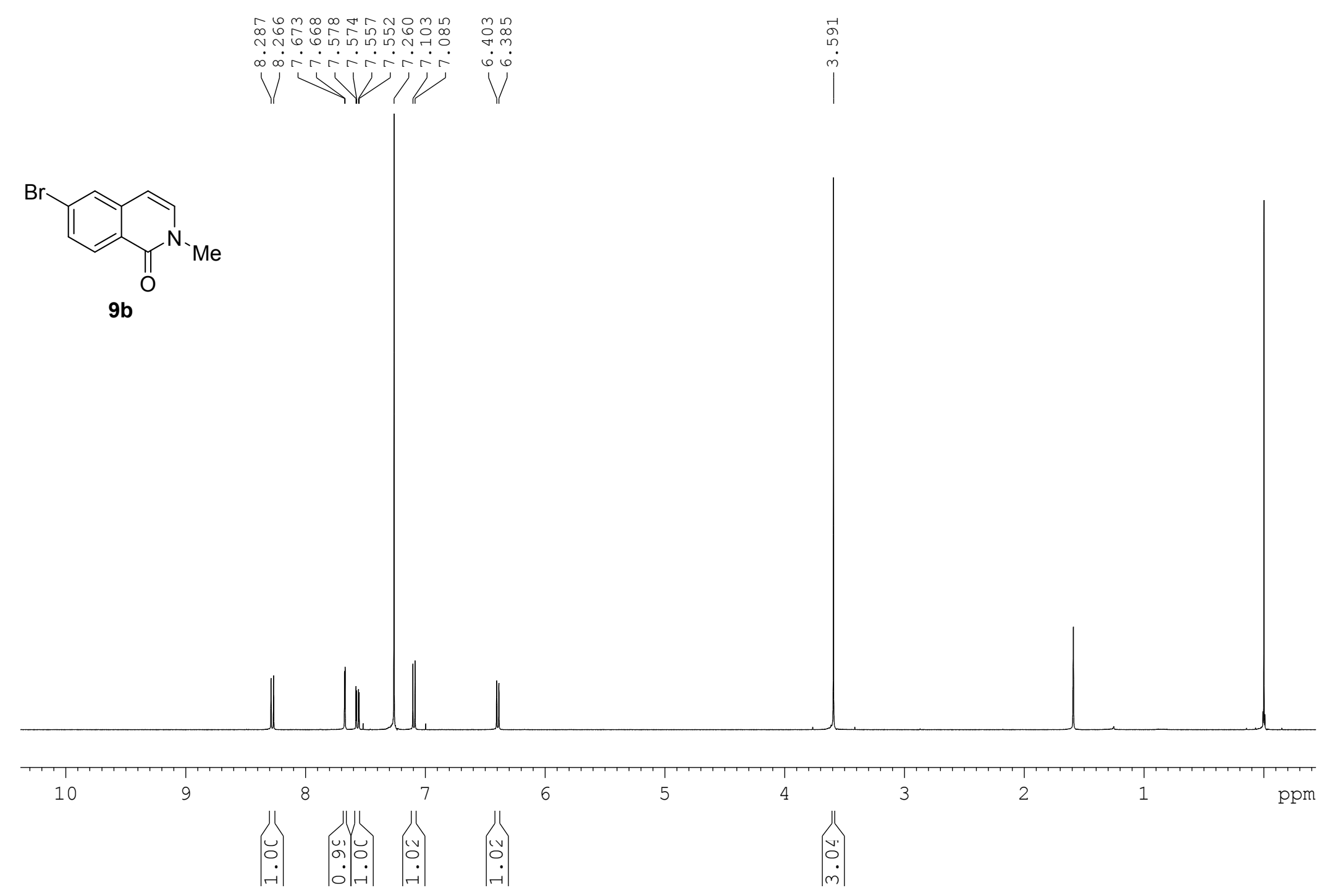

Figure S47. ${ }^{1} \mathrm{H}$ NMR spectrum of $9 b\left(\mathrm{CDCl}_{3}, 400 \mathrm{MHz}\right)$. 


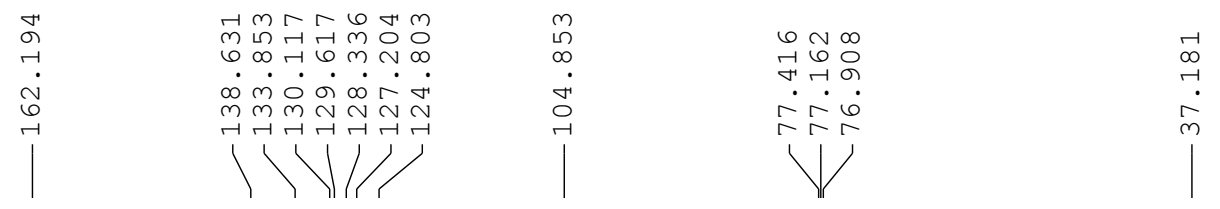

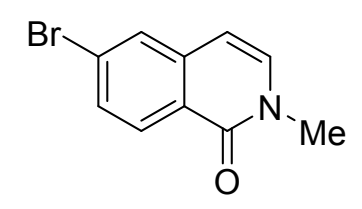

$9 b$

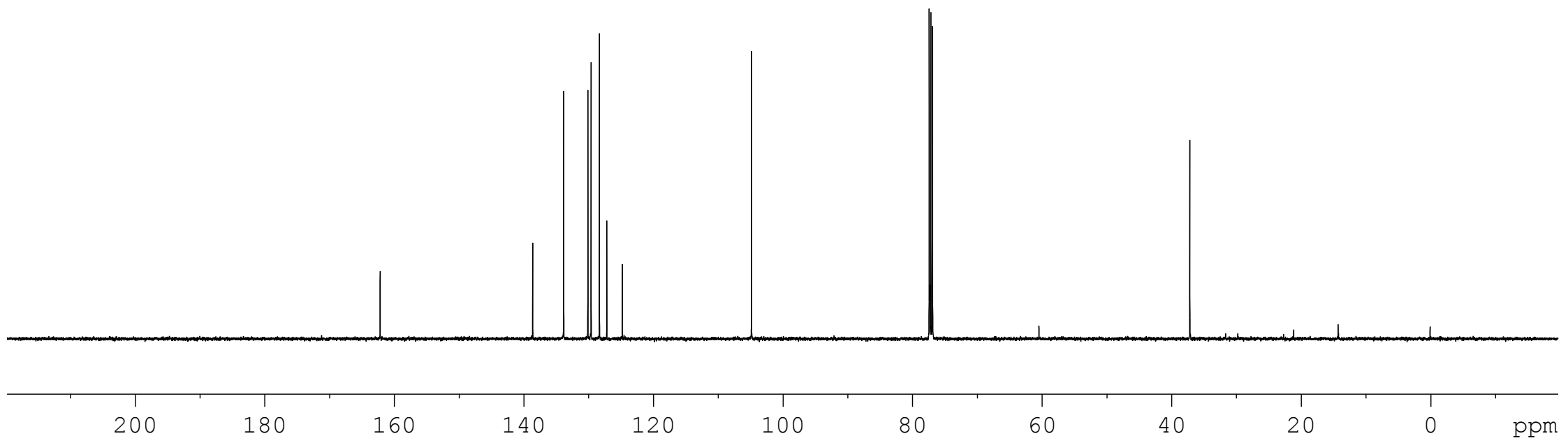

Figure $\mathbf{S 4 8 .}{ }^{13} \mathrm{C}$ NMR spectrum of $\mathbf{9 b}\left(\mathrm{CDCl}_{3}, 100 \mathrm{MHz}\right)$. 

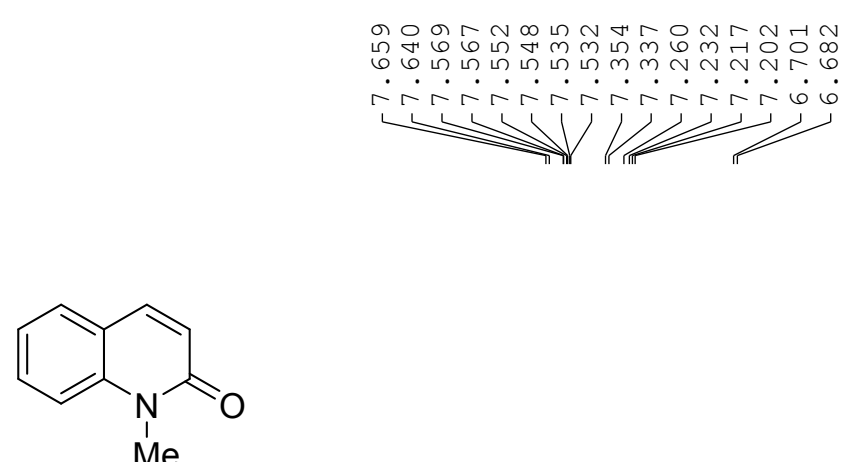

9c

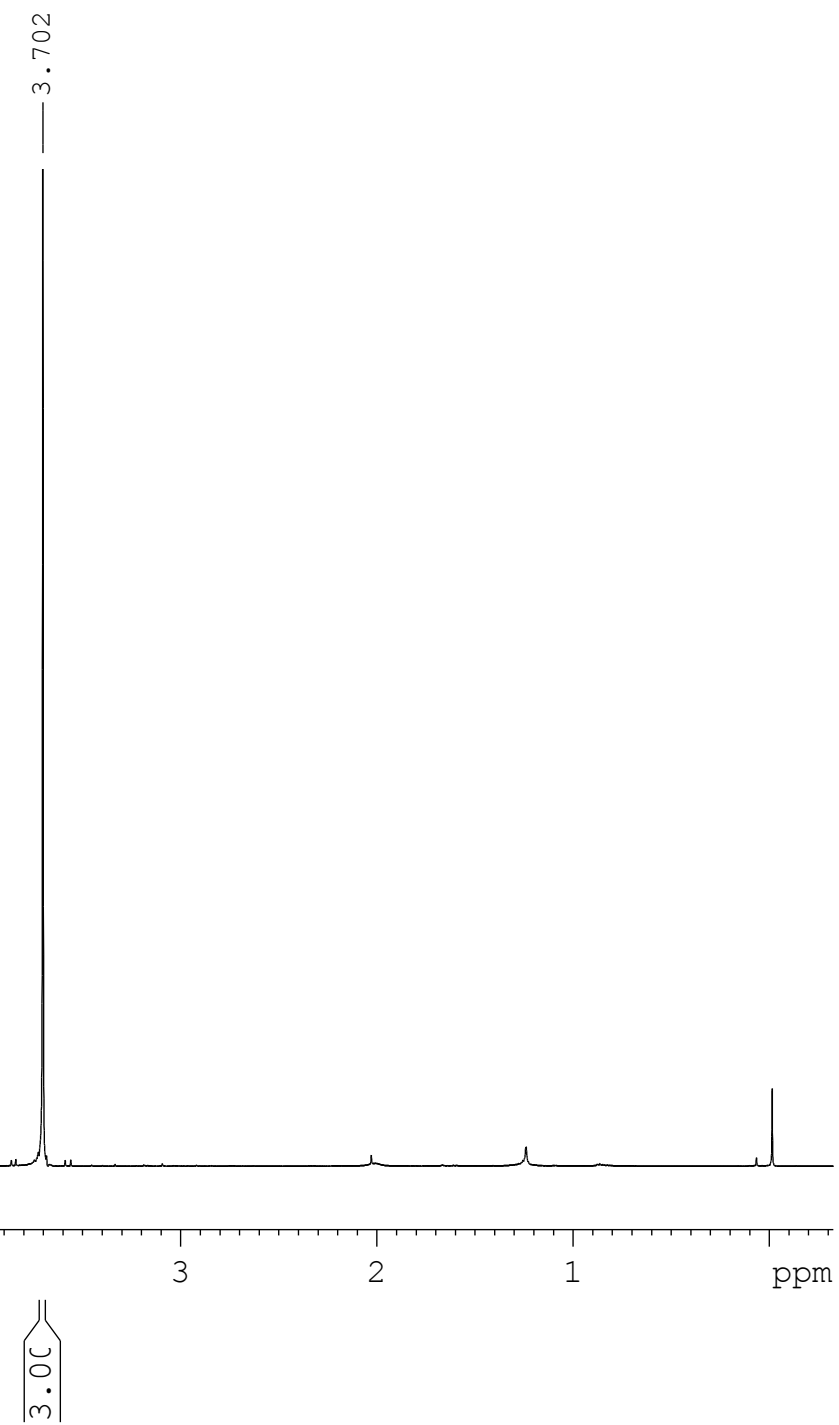

Figure S49. ${ }^{1} \mathrm{H}$ NMR spectrum of $9 c\left(\mathrm{CDCl}_{3}, 500 \mathrm{MHz}\right)$. 

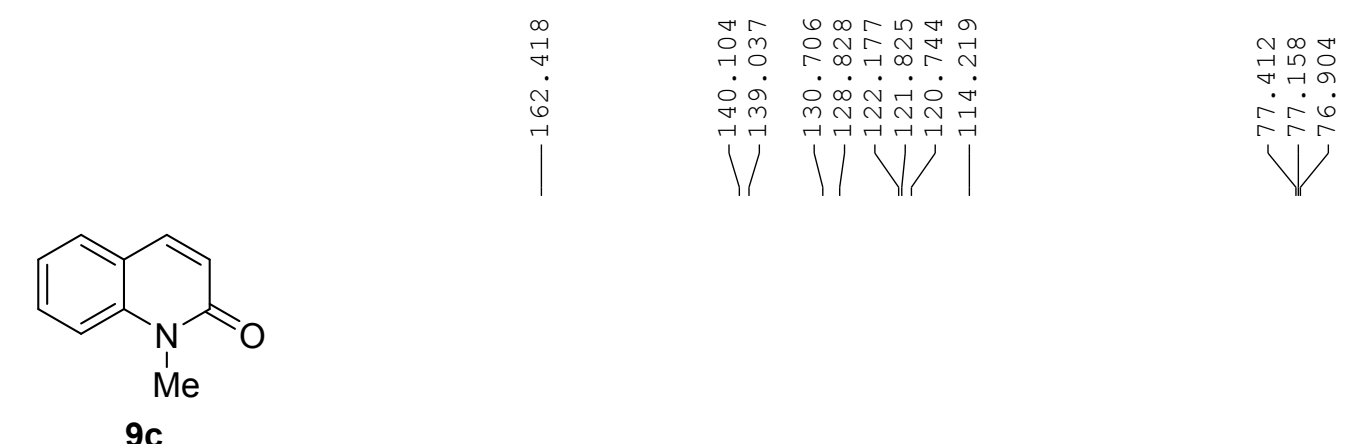

9c

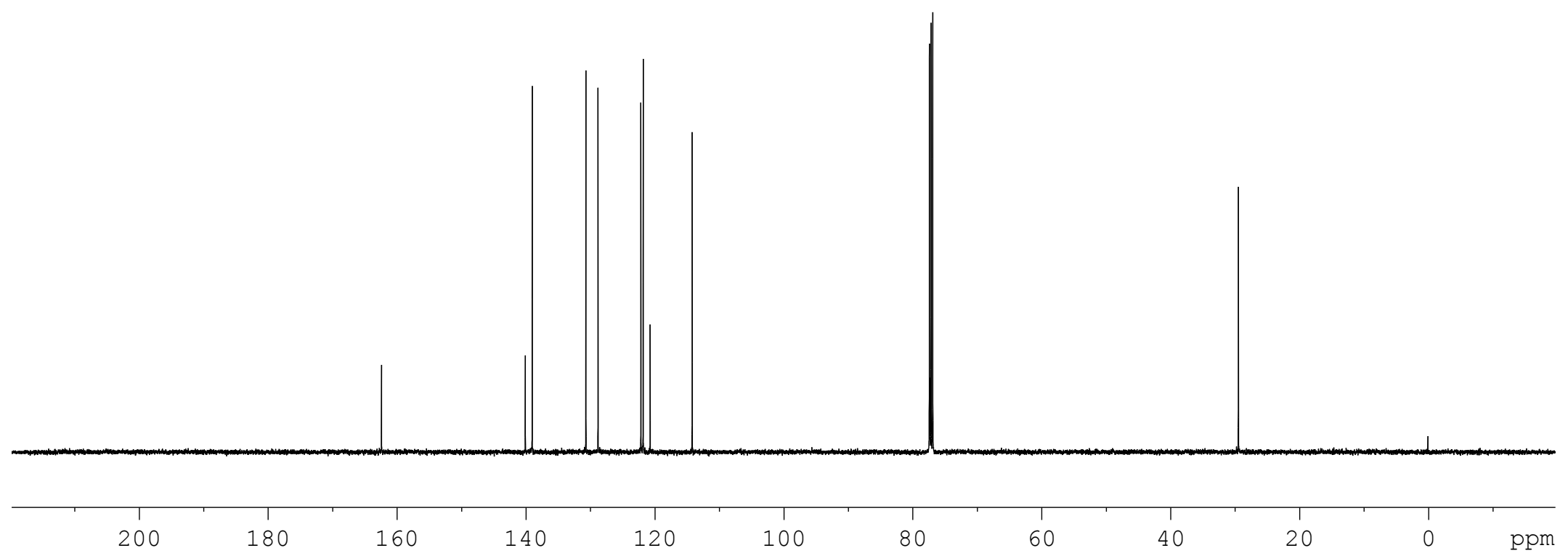

Figure S50. ${ }^{13} \mathrm{C}$ NMR spectrum of $9 c\left(\mathrm{CDCl}_{3}, 125 \mathrm{MHz}\right)$. 


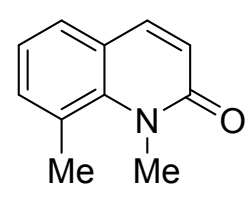

9d

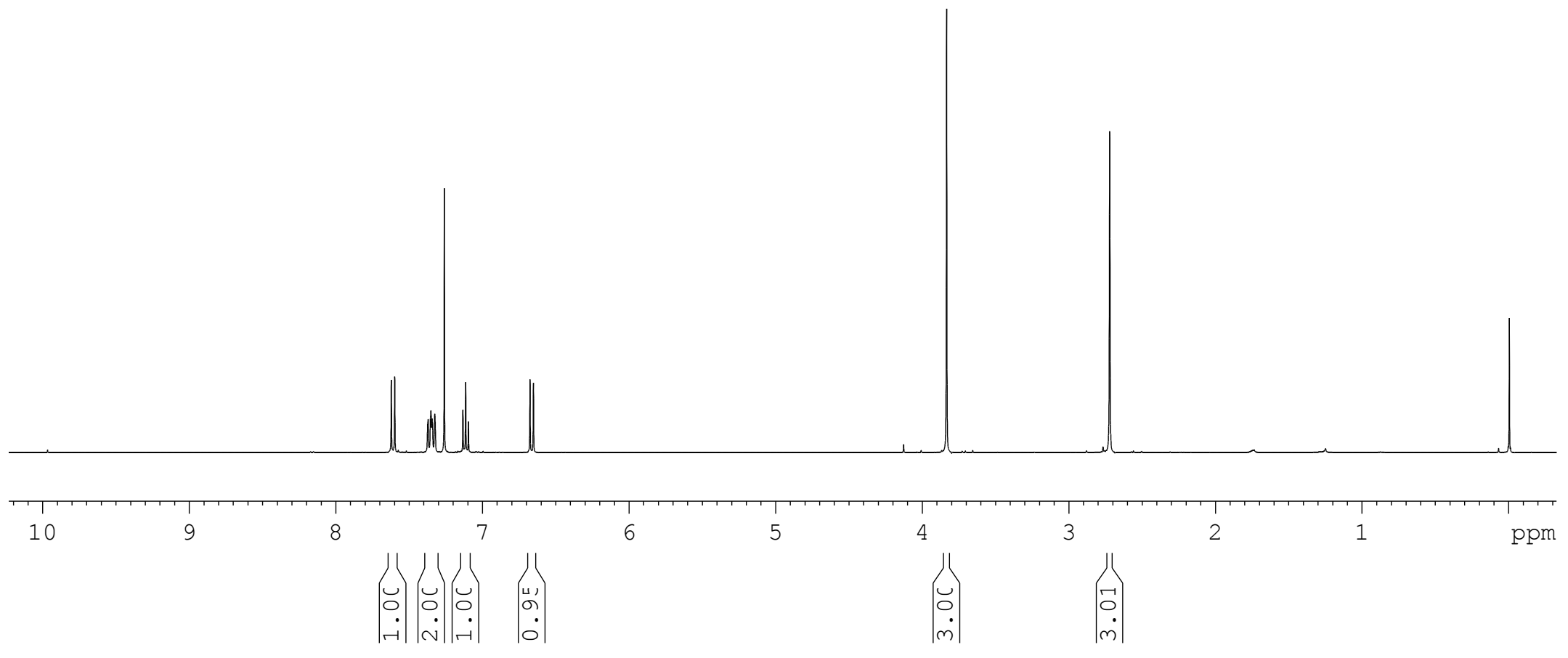

Figure S51. ${ }^{1} \mathrm{H}$ NMR spectrum of $9 d\left(\mathrm{CDCl}_{3}, 400 \mathrm{MHz}\right)$. 


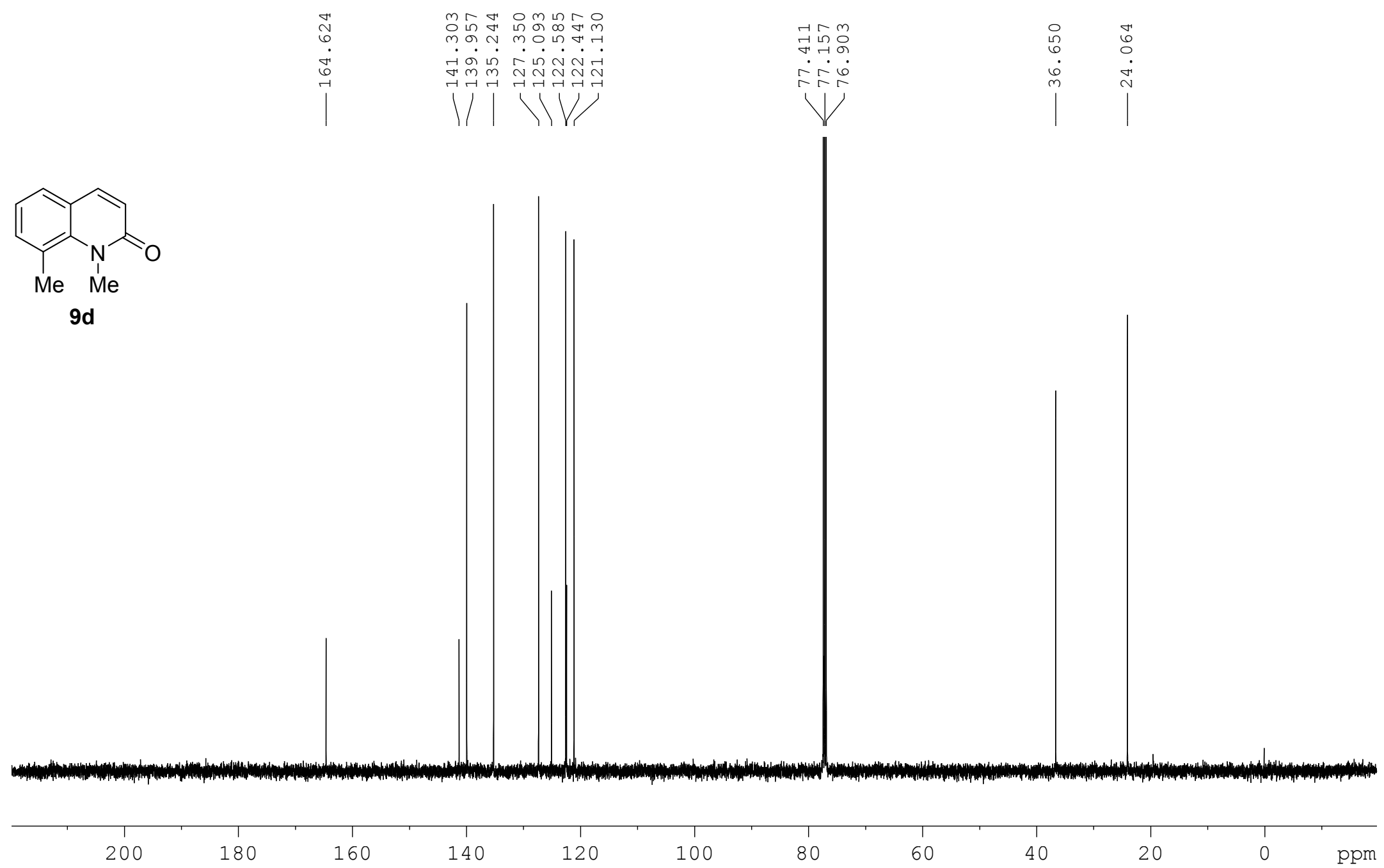

Figure S52. ${ }^{13} \mathrm{C}$ NMR spectrum of $\mathbf{9 d}\left(\mathrm{CDCl}_{3}, 100 \mathrm{MHz}\right)$. 


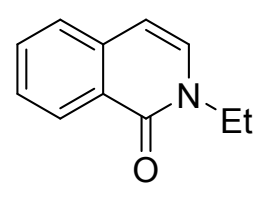

$9 e$

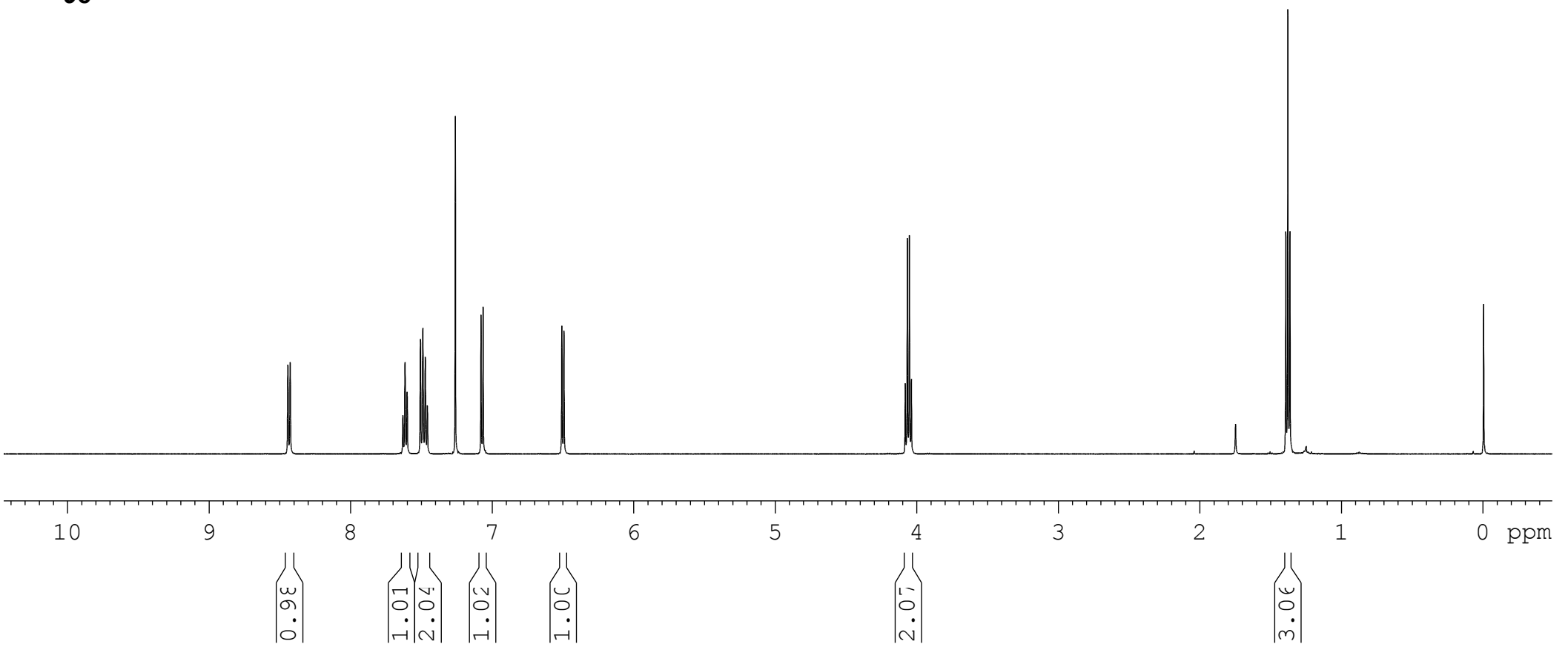

Figure S53. ${ }^{1} \mathrm{H}$ NMR spectrum of $9 \mathrm{e}\left(\mathrm{CDCl}_{3}, 500 \mathrm{MHz}\right)$ 


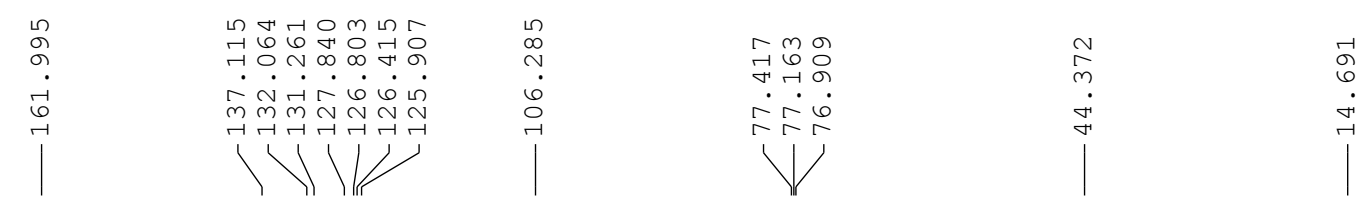

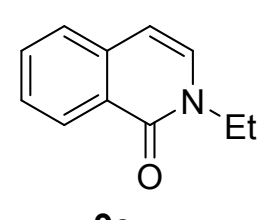

$9 e$

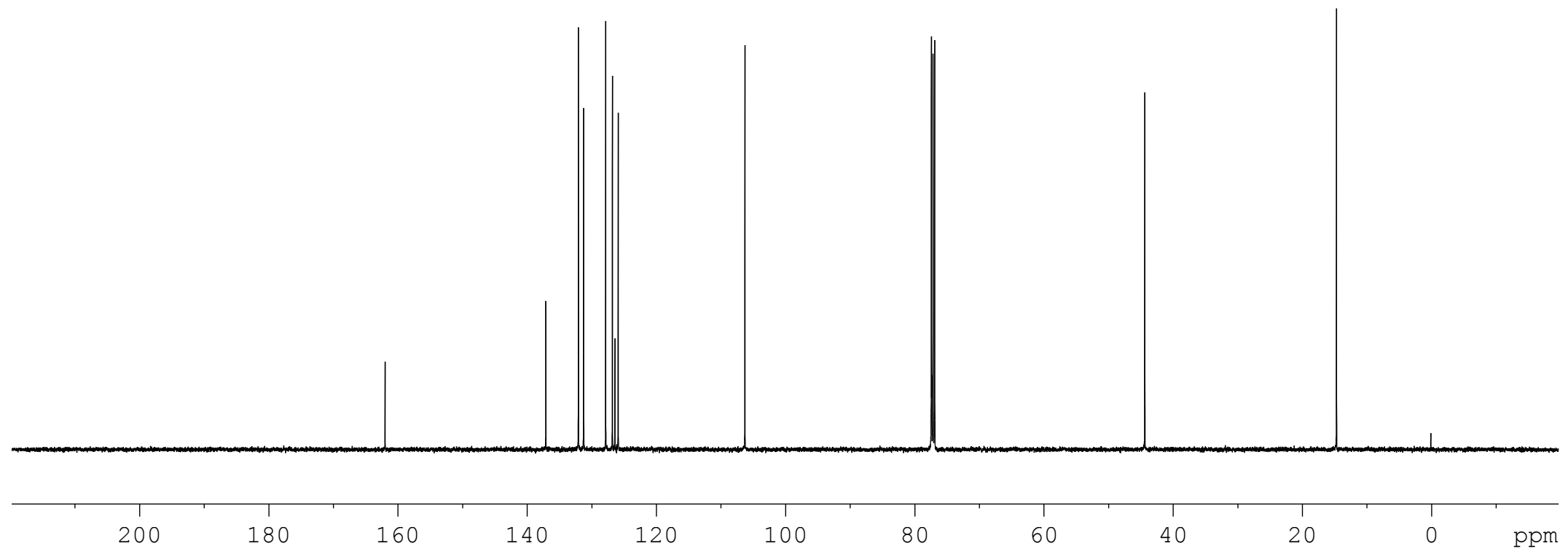

Figure S54. ${ }^{13} \mathrm{C}$ NMR spectrum of $9 e\left(\mathrm{CDCl}_{3}, 125 \mathrm{MHz}\right)$. 

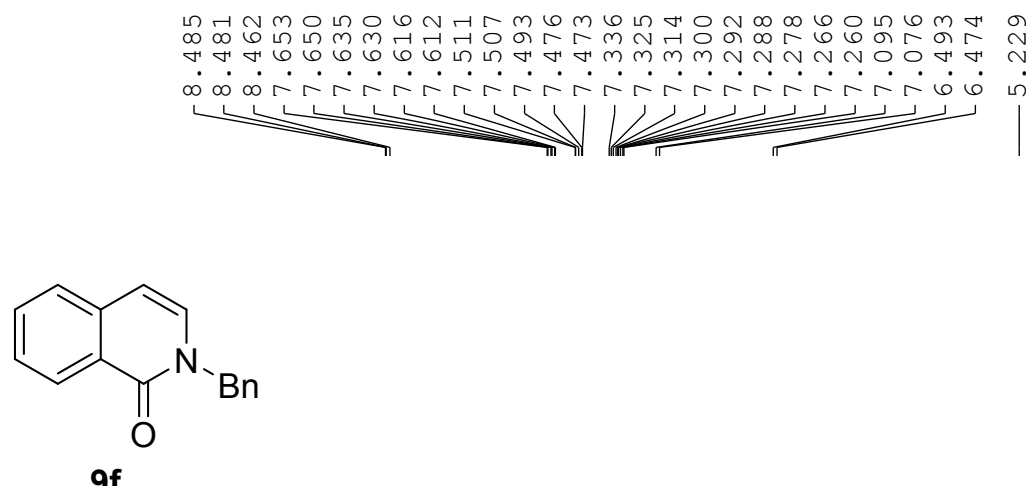

$9 f$

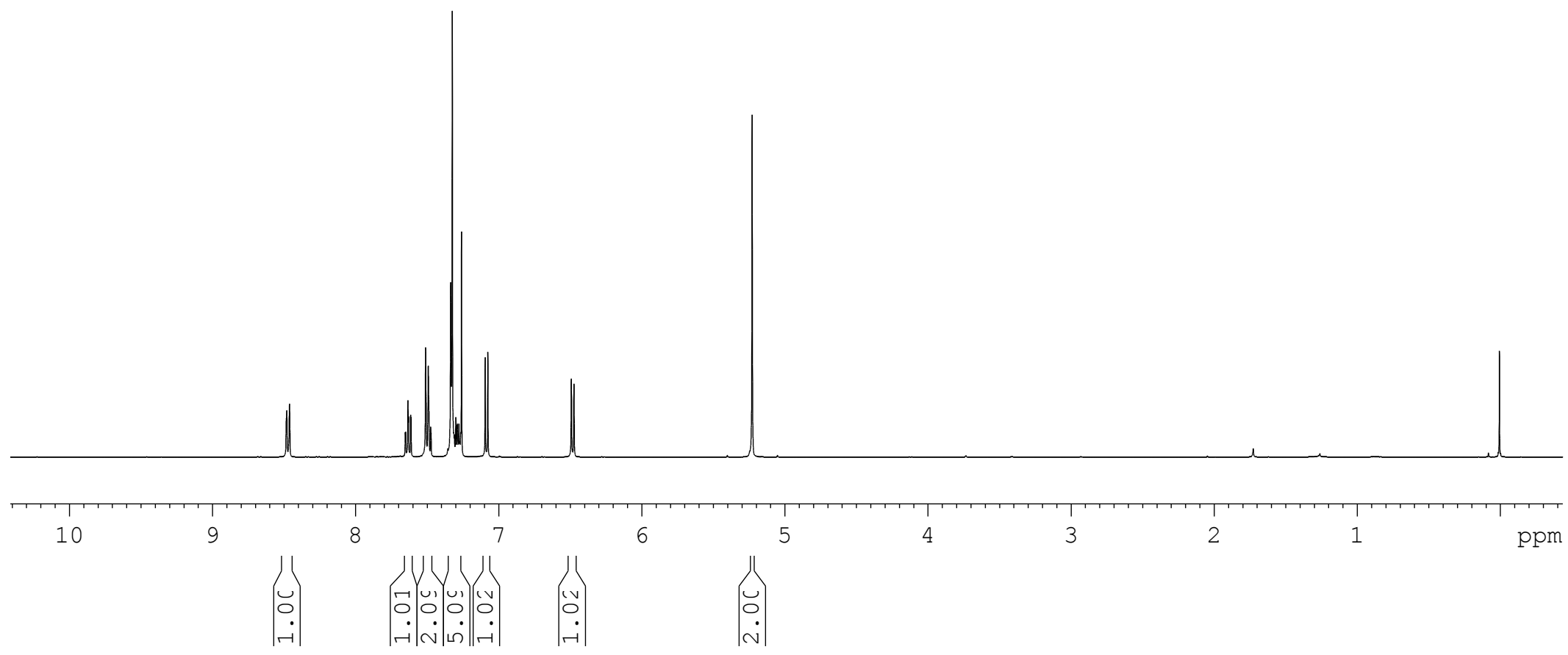

Figure S55. ${ }^{1} \mathrm{H}$ NMR spectrum of $9 f\left(\mathrm{CDCl}_{3}, 400 \mathrm{MHz}\right)$. 

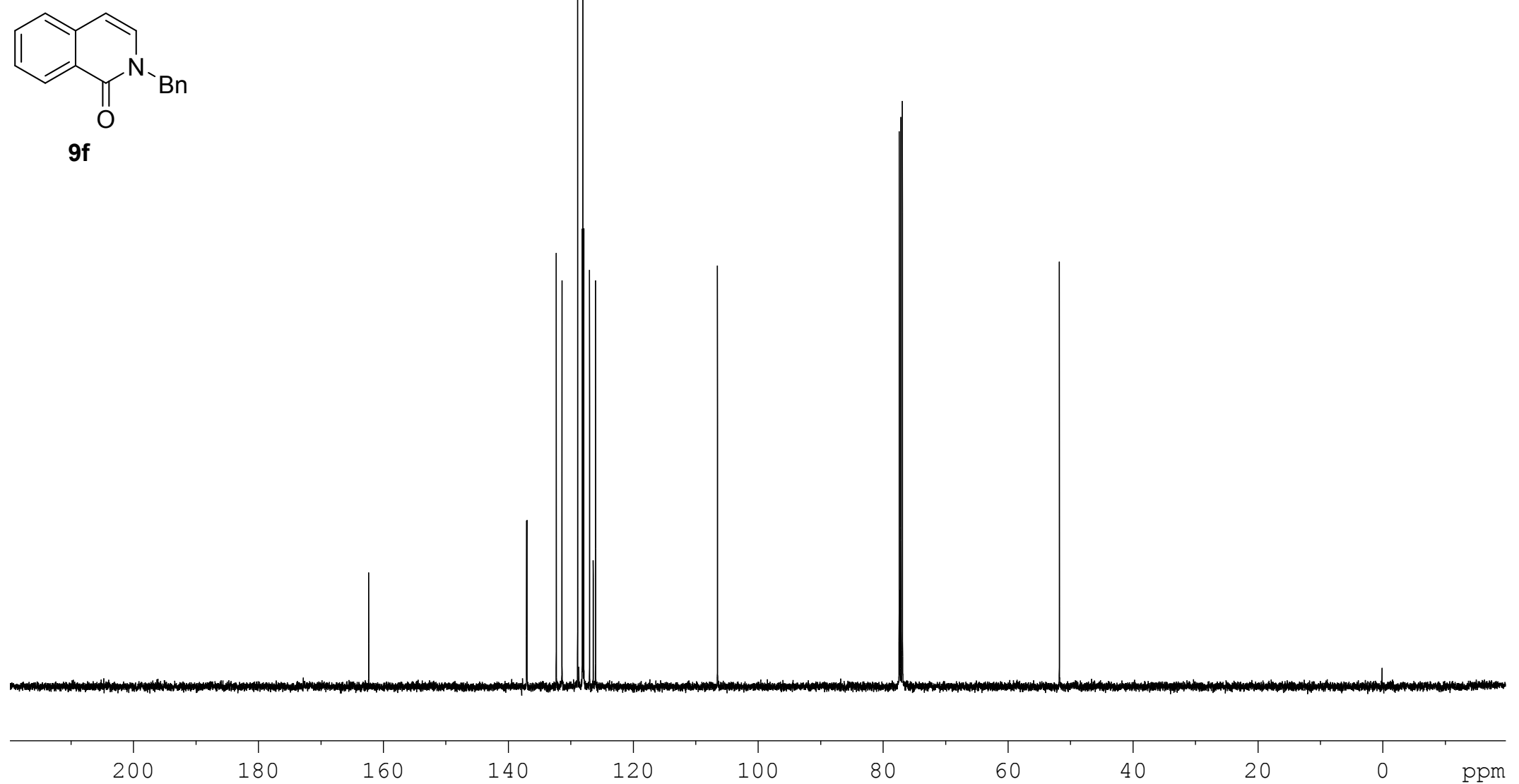

Figure S56. ${ }^{13} \mathrm{C}$ NMR spectrum of $\mathbf{9 f}\left(\mathrm{CDCl}_{3}, 100 \mathrm{MHz}\right)$. 


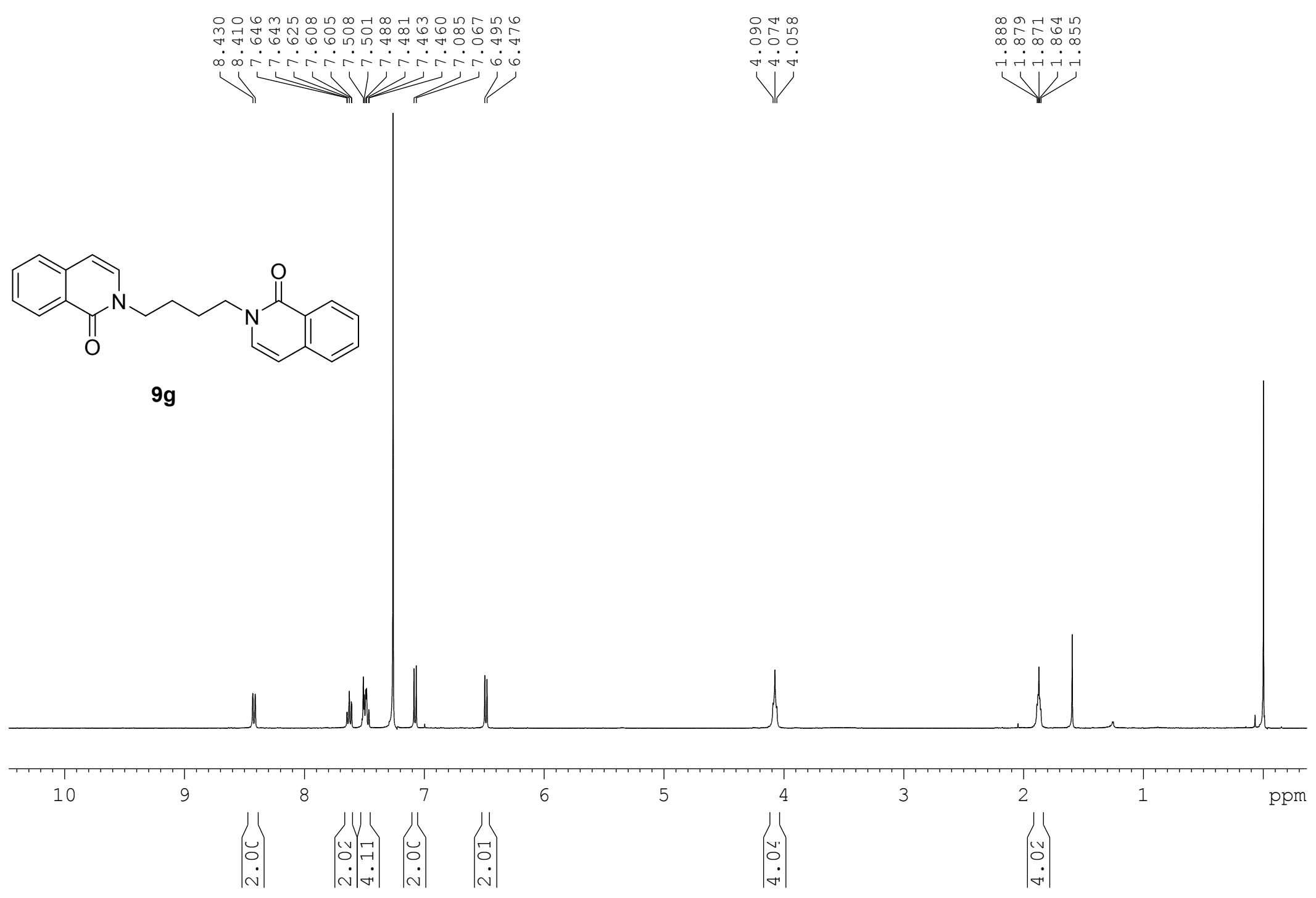

Figure S57. ${ }^{1} \mathrm{H}$ NMR spectrum of $9 \mathrm{~g}\left(\mathrm{CDCl}_{3}, 400 \mathrm{MHz}\right)$. 


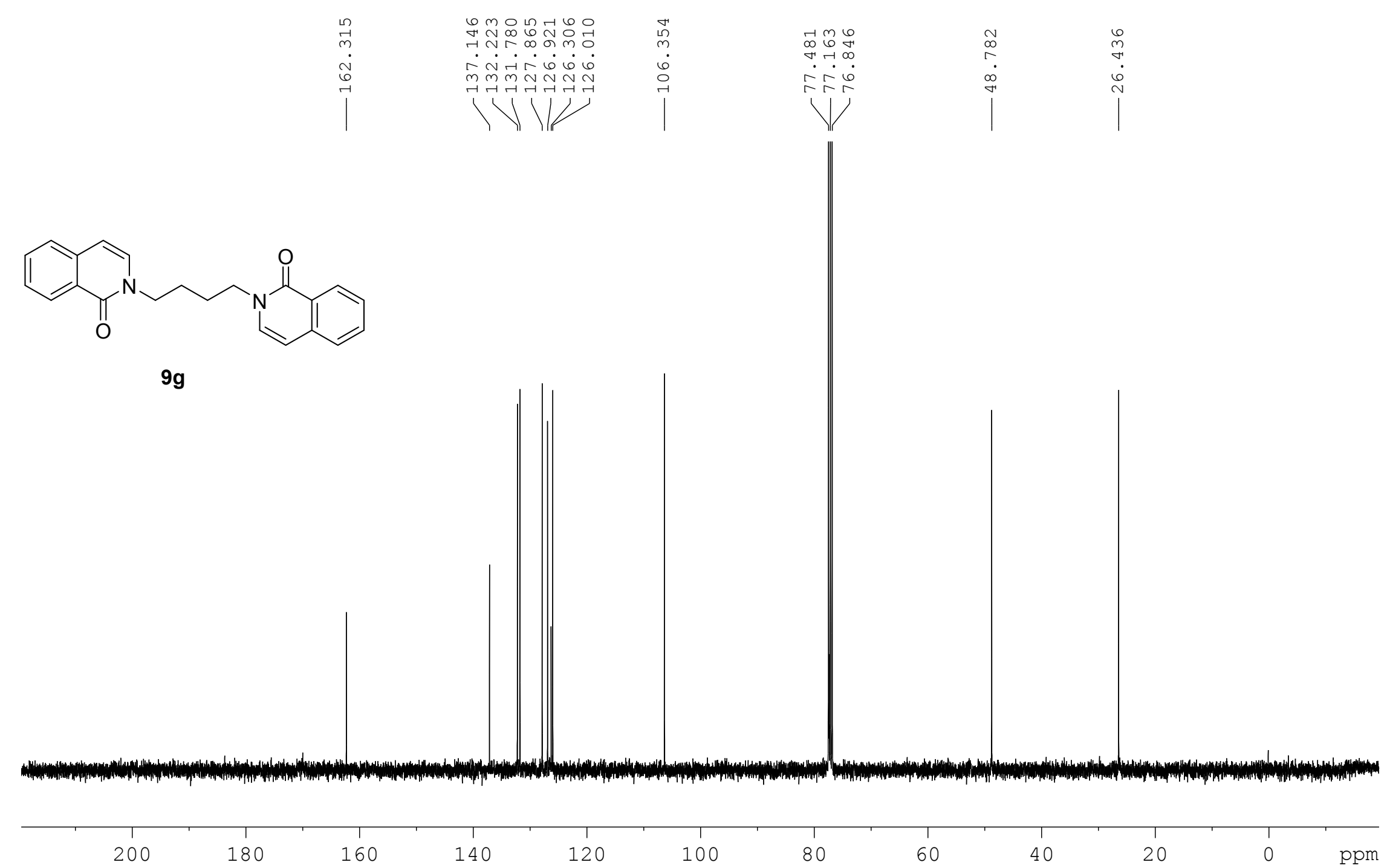

Figure S58. ${ }^{13} \mathrm{C}$ NMR spectrum of $9 \mathrm{~g}\left(\mathrm{CDCl}_{3}, 100 \mathrm{MHz}\right)$. 